\title{
Bôcher and Abstract Contractions of 2nd Order Quadratic Algebras
}

\author{
Mauricio A. ESCOBAR RUIZ $\dagger^{\dagger^{1} \dagger^{2}}$, Ernest G. KALNINS $\dagger^{3}$, Willard MILLER Jr. $\dagger^{2}$ \\ and Eyal $S U B A G^{\dagger^{4}}$ \\ $\dagger^{1}$ Instituto de Ciencias Nucleares, UNAM, Apartado Postal 70-543, 04510 Mexico D.F. Mexico \\ E-mail: mauricio.escobar@nucleares.unam.mx \\ $\dagger^{2}$ School of Mathematics, University of Minnesota, Minneapolis, Minnesota, 55455, USA \\ E-mail:miller@ima.umn.edu \\ URL: https://www.ima.umn.edu/ miller/ \\ $\dagger^{3}$ Department of Mathematics, University of Waikato, Hamilton, New Zealand \\ E-mail: math0236@waikato.ac.nz \\ $\dagger^{4}$ Department of Mathematics, Pennsylvania State University, State College, \\ Pennsylvania, 16802, USA \\ E-mail: eyalsubag@gmail.com
}

Received November 19, 2016, in final form February 27, 2017; Published online March 06, 2017

https://doi.org/10.3842/SIGMA.2017.013

\begin{abstract}
Quadratic algebras are generalizations of Lie algebras which include the symmetry algebras of 2 nd order superintegrable systems in 2 dimensions as special cases. The superintegrable systems are exactly solvable physical systems in classical and quantum mechanics. Distinct superintegrable systems and their quadratic algebras can be related by geometric contractions, induced by Bôcher contractions of the conformal Lie algebra $\mathfrak{s o}(4, \mathbb{C})$ to itself. In this paper we give a precise definition of Bôcher contractions and show how they can be classified. They subsume well known contractions of $\mathfrak{e}(2, \mathbb{C})$ and $\mathfrak{s o}(3, \mathbb{C})$ and have important physical and geometric meanings, such as the derivation of the Askey scheme for obtaining all hypergeometric orthogonal polynomials as limits of Racah/Wilson polynomials. We also classify abstract nondegenerate quadratic algebras in terms of an invariant that we call a canonical form. We describe an algorithm for finding the canonical form of such algebras. We calculate explicitly all canonical forms arising from quadratic algebras of 2D nondegenerate superintegrable systems on constant curvature spaces and Darboux spaces. We further discuss contraction of quadratic algebras, focusing on those coming from superintegrable systems.
\end{abstract}

Key words: contractions; quadratic algebras; superintegrable systems; conformal superintegrability

2010 Mathematics Subject Classification: 22E70; 16G99; 37J35; 37K10; 33C45; 17B60; 81R05; 33C45

\section{Introduction}

Second order 2D superintegrable systems and their associated quadratic symmetry algebras are basic in mathematical physics. Among the simplest such solvable systems are the 2D Kepler and hydrogen atom and the isotropic and Higgs oscillators [30, 34]. All the systems are multiseparable, with the quantum separable solutions characterized as eigenfunctions of commuting operators in the quadratic algebras. The separation equations are the Gaussian hypergeometric equation and its various confluent forms in full generality, as well as the Heun equation and its confluent forms in full generality [5]. Solutions of the hypergeometric and Heun equations are 
linked through their solution of the same superintegrable system. The confluences are related to Bôcher contractions of the conformal algebra $\mathfrak{s o}(4, \mathbb{C})$ to itself [27]. The interbasis expansion coefficients relating distinct separable systems lead to other special functions, several of them functions of discrete variables, such as the Racah, Wilson and Hahn polynomials in full generality [25]. The contractions also allow the derivation of the Askey scheme for the classification of hypergeometric orthogonal polynomials. The classification of quasi-exactly solvable (QES) systems based on the Heun operator coincide exactly with QES separation equations for these superintegrable systems $[35,36]$.

In short, the structure and classification of these quadratic algebras and their relations via contractions are matters of considerable significance in mathematical physics. Historically, the superintegrable systems have been classified and their associated quadratic algebras then computed. Here we are reversing the process: we first classify abstract quadratic algebras and then determine which of these correspond to 2nd order superintegrable systems. Also we determine how the abstract quadratic algebras are related via contractions and examine which of these contractions can be realized geometrically as Bôcher contractions. The eventual goal is to isolate the algebras and contractions that do not correspond to geometrical superintegrable systems and to determine their significance.

Bôcher invented a recipe for a limit procedure which showed how to find what we now know are all $R$-separable coordinate systems for free Laplace and wave equations in $n$ dimensions [1]. We have recently recognized that these limits can be interpreted as contractions of $\mathfrak{s o}(n+2, \mathbb{C})$ to itself and classified; we call them Bôcher contractions. In this paper we give for the first time the precise definition of these contractions and their properties and classification for the case $n=2$.

We start with some basic facts. We define a quantum (Helmholtz) superintegrable system as an integrable Hamiltonian system on an $n$-dimensional pseudo-Riemannian manifold with potential: $H=\Delta_{n}+V$ that admits $2 n-1$ algebraically independent partial differential operators $L_{j}$ commuting with $H$, the maximum possible: $\left[H, L_{j}\right]=0, j=1,2, \ldots, 2 n-1$. Similarly a classical superintegrable on such a manifold, with Hamiltonian $\mathcal{H}=\sum g^{i j} p_{i} p_{j}+V$, is an integrable system that admits $2 n-1$ functionally independent constants of the motion $\mathcal{L}_{j}$, polynomial in the momenta, in involution with $\mathcal{H}$, the maximum possible. Superintegrability captures the properties of quantum Hamiltonian systems that allow the Schrödinger eigenvalue problem (or Helmholtz equation) $H \Psi=E \Psi$ to be solved exactly, analytically and algebraically [7, 8, 30, 33, 34] and the classical trajectories to be computed algebraically. A system is of order $K$ if the maximum order of the symmetry operators (or the polynomial order of the classical constants of the motion), other than $H$, is $K$. For $n=2, K=1,2$ all systems are known, e.g., [4, 14, 15, 16, 17, 18, 19]. For $K=1$ the symmetry algebras are just Lie algebras.

We review briefly the facts for free 2 nd order superintegrable systems (i.e., no potential, $K=2$ ) in the case $n=2,2 n-1=3$. The complex spaces with Laplace-Beltrami operators admitting at least three 2nd order symmetries were classified by Koenigs (1896) [28]. They are: the two constant curvature spaces (flat space and the complex sphere), the four Darboux spaces (one of which, D4, contains a parameter) [21], and 5 families of 4-parameter Koenigs spaces, see Section 1.1. For 2nd order systems with non-constant potential the generating symmetry operators of each system close under commutation (or via Poisson brackets in the classical case) to determine a quadratic algebra, and the irreducible representations of the quantum algebra determine the eigenvalues of $H$ and their multiplicities. More precisely, in the classical case, closedness means that the Poisson algebra generated by the constants of motion is finitely generated as an associative algebra. The quantum case is defined analogously. Here we consider only the nondegenerate superintegrable systems: Those with 4-parameter potentials (including the additive constant) (the maximum possible):

$$
V(\mathbf{x})=a_{1} V_{(1)}(\mathbf{x})+a_{2} V_{(2)}(\mathbf{x})+a_{3} V_{(3)}(\mathbf{x})+a_{4}
$$


where $\left\{V_{(1)}(\mathbf{x}), V_{(2)}(\mathbf{x}), V_{(3)}(\mathbf{x}), 1\right\}$ is a linearly independent set. Here the possible classical and quantum potentials are identical and there is a 1-1 relationship between classical and quantum systems. The classical constants of the motion determine the quantum symmetry operators, modulo symmetrization. The classical symmetry algebra generated by $\mathcal{H}, \mathcal{L}_{1}, \mathcal{L}_{2}$ always closes under commutation and gives the following nondegenerate quadratic algebra structure:

Definition 1.1. An abstract nondegenerate (classical) quadratic algebra is a Poisson algebra with functionally independent generators $\mathcal{H}, \mathcal{L}_{1}, \mathcal{L}_{2}$, and parameters $a_{1}, a_{2}, a_{3}, a_{4}$, such that all generators are in involution with $\mathcal{H}$ and the following relations hold:

$$
\begin{aligned}
& \left\{\mathcal{L}_{j}, \mathcal{R}\right\}=\sum_{0 \leq e_{1}+e_{2}+e_{3} \leq 2} M_{e_{1}, e_{2}, e_{3}}^{(j)} \mathcal{L}_{1}^{e_{1}} \mathcal{L}_{2}^{e_{2}} \mathcal{H}^{e_{3}}, \quad e_{k} \geq 0, \quad \mathcal{L}_{k}^{0}=1, \\
& \mathcal{R}^{2}=\mathcal{F} \equiv \sum_{0 \leq e_{1}+e_{2}+e_{3} \leq 3} N_{e_{1}, e_{2}, e_{3}} \mathcal{L}_{1}^{e_{1}} \mathcal{L}_{2}^{e_{2}} \mathcal{H}^{e_{3}}
\end{aligned}
$$

Here, $\mathcal{R} \equiv\left\{\mathcal{L}_{1}, \mathcal{L}_{2}\right\}$. In both equations the constants $M_{e_{1}, e_{2}, e_{3}}^{(j)}$ and $N_{e_{1}, e_{2}, e_{3}}$ are polynomials in the parameters $a_{1}, a_{2}, a_{3}$ of degree $2-e_{1}-e_{2}-e_{3}$ and $3-e_{1}-e_{2}-e_{3}$, respectively. The symmetry algebras obeyed by the quantum superintegrable systems have a similar structure, slightly more complicated due to the need for symmetrization of the noncommuting operators. In the case $a_{1}=a_{2}=a_{3}=a_{4}=0$, the corresponding quadratics algebras are called free.

Note that we can think of a nondegenerate (classical or quantum) quadratic algebra as a family of algebras parametrized by the constants $a_{i}$. The algebra is called quadratic because the Poisson brackets $\left\{\mathcal{L}_{j}, \mathcal{R}\right\}$ are 2 nd order polynomials in the generators $\mathcal{L}_{i}, \mathcal{H}$, whereas for a Lie algebra they are 1 st order. Nondegenerate $2 \mathrm{D}$ superintegrable systems always have a quadratic algebra structure in which the parameters $a_{j}$ are those of the potential; we call these quadratic algebras geometrical.

Although the full sets of classical structure equations can be rather complicated, the function $\mathcal{F}$ contains all of the structure information for nondegenerate systems. In particular, it is easy to show that, e.g., [23],

$$
\left\{\mathcal{L}_{1}, \mathcal{R}\right\}=\frac{1}{2} \frac{\partial \mathcal{F}}{\partial \mathcal{L}_{2}}, \quad\left\{\mathcal{L}_{2}, \mathcal{R}\right\}=-\frac{1}{2} \frac{\partial \mathcal{F}}{\partial \mathcal{L}_{1}},
$$

for any algebra satisfying Definition 1.1 , so $\mathcal{F}$ determines the structure equations explicitly.

For a nondegenerate superintegrable system with potential (1.1) the structure equations are determined by $\mathcal{F}\left(\mathcal{H}, \mathcal{L}_{1}, \mathcal{L}_{2}, a_{1}, a_{2}, a_{3}, a_{4}\right)$ as defined above. The effect of a Stäckel transform [24] generated by the specific special choice of the potential function, say $V_{(3)}$ is to determine a new superintegrable system with Casimir $\tilde{\mathcal{R}}^{2}=\mathcal{F}\left(-a_{3}, \mathcal{L}_{1}, \mathcal{L}_{2}, a_{1}, a_{2},-\mathcal{H}, a_{4}\right)$. Of course, the switch of $a_{3}$ and $\mathcal{H}$ is only for illustration; there is a Stäckel transform that replaces any $a_{j}$ by $-\mathcal{H}$ and $\mathcal{H}$ by $-a_{j}$ and similar transforms that apply to any basis that we choose for the potential space.

If we consider the free systems (zero potential which is the case with all parameters equal zero) on the spaces classified by Koenigs, then the vector space of 2nd order symmetries may be larger than 3: 6-dimensional for constant curvature spaces, 4-dimensional for Darboux spaces, and 3-dimensional for Koenigs spaces. In general the Poisson algebras generated by taking Poisson brackets of these 2nd order elements are infinite-dimensional; they do not close (in the sense that was explained above). However, in [23], the possible 3-dimensional subspaces of 2nd order free symmetries that generate quadratic algebras were classified, up to conjugacy by symmetry groups of these spaces: $\mathfrak{e}(2, \mathbb{C})$ for flat space, $\mathfrak{o}(3, \mathbb{C})$ for nonzero constant curvature spaces, and a 1-dimensional translation subalgebra for Darboux spaces. For Koenigs spaces the first order symmetry algebra is 0-dimensional and the space of 2 nd order symmetries is 3 -dimensional which always generates a unique quadratic algebra. 
Theorem 1.2. For each of the spaces classified by Koenigs, there is a bijection between free quadratic algebras of 2 nd order symmetries, classified up to conjugacy, and 2 nd order nondegenerate superintegrable systems on these spaces.

The proof of this theorem is constructive [23]. Given a free quadratic algebra $\tilde{Q}$ one can compute the potential $V$ and the symmetries of the quadratic algebra $Q$ of the nondegenerate superintegrable system. (The quadratic algebra structure guarantees that the Bertrand-Darboux equations for the potential are satisfied identically. In this sense the free systems "know" the possible nondegenerate superintegrable systems they can support. Since there is a 1-1 relationship between quantum and classical nondegenerate systems, the information about all of these systems is encoded in the free quadratic algebras generated by 2 nd order constants of the motion (Killing tensors) of constant curvature, Darboux and Koenigs spaces. Note that for flat space the generators for the free quadratic algebras can be expressed as 2nd order elements in the universal enveloping algebra of $\mathfrak{e}(2, \mathbb{C})$, and for nonzero constant curvature spaces the generators for the free quadratic algebras can be expressed as 2nd order elements in the universal enveloping algebra of $\mathfrak{s o}(3, \mathbb{C})[23]$.

All 2nd order 2D superintegrable systems with potential and their quadratic algebras are known. There are 33 nondegenerate systems, on a variety of manifolds classified up to conjugacy, see Section 1.1 where the numbering for constant curvature systems is taken from [22], (the numbers are not always consecutive because the lists in [22] also include degenerate systems) and the numbering for Darboux spaces is taken from [21]. For each system we give the 4-parameter potential and the abstract free structure equation $\mathcal{R}^{2}-\mathcal{F}=0$. Note that many of the abstract structure equations for the superintegrable systems are identical, even for superintegrable systems on different manifolds. Of course the geometrical structure equations are distinct because the generators $\mathcal{L}_{1}, \mathcal{L}_{2}, \mathcal{H}$ are distinct for each geometrical superintegrable system.

Under the Stäckel transform (we discuss this in Section 2.1) these systems divide into 6 equivalence classes with representatives on flat space and the 2-sphere, see [29] and Section 3.3.

\subsection{The Helmholtz nondegenerate superintegrable systems}

Flat space systems: $\mathcal{H} \equiv p_{x}^{2}+p_{y}^{2}+V=E$.

1. $E 1: V=\alpha\left(x^{2}+y^{2}\right)+\frac{\beta}{x^{2}}+\frac{\gamma}{y^{2}}, \mathcal{R}^{2}=\mathcal{L}_{1} \mathcal{L}_{2}\left(\mathcal{H}+\mathcal{L}_{2}\right)$,

2. $E 2: V=\alpha\left(4 x^{2}+y^{2}\right)+\beta x+\frac{\gamma}{y^{2}}, \mathcal{R}^{2}=\mathcal{L}_{1}^{2}\left(\mathcal{H}+\mathcal{L}_{1}\right)$,

3. $E 3^{\prime}: V=\alpha\left(x^{2}+y^{2}\right)+\beta x+\gamma y, \quad \mathcal{R}^{2}=0$,

4. $E 7: V=\frac{\alpha(x+i y)}{\sqrt{(x+i y)^{2}-b}}+\frac{\beta(x-i y)}{\sqrt{(x+i y)^{2}-b}\left(x+i y+\sqrt{(x+i y)^{2}-b}\right)^{2}}+\gamma\left(x^{2}+y^{2}\right), \mathcal{R}^{2}=\mathcal{L}_{1} \mathcal{L}_{2}^{2}+b \mathcal{L}_{2} \mathcal{H}^{2}$,

5. $E 8: V=\frac{\alpha(x-i y)}{(x+i y)^{3}}+\frac{\beta}{(x+i y)^{2}}+\gamma\left(x^{2}+y^{2}\right), \mathcal{R}^{2}=\mathcal{L}_{1} \mathcal{L}_{2}^{2}$,

6. $E 9: V=\frac{\alpha}{\sqrt{x+i y}}+\beta y+\frac{\gamma(x+2 i y)}{\sqrt{x+i y}}, \mathcal{R}^{2}=\mathcal{L}_{1}\left(\mathcal{L}_{1}+\mathcal{H}\right)^{2}$,

7. $E 10: V=\alpha(x-i y)+\beta\left(x+i y-\frac{3}{2}(x-i y)^{2}\right)+\gamma\left(x^{2}+y^{2}-\frac{1}{2}(x-i y)^{3}\right), \mathcal{R}^{2}=\mathcal{L}_{1}^{3}$,

8. $E 11: V=\alpha(x-i y)+\frac{\beta(x-i y)}{\sqrt{x+i y}}+\frac{\gamma}{\sqrt{x+i y}}, \mathcal{R}^{2}=\mathcal{L}_{1} \mathcal{H}^{2}$,

9. $E 15: V=f(x-i y)$, where $f$ is arbitrary, $\mathcal{R}^{2}=\mathcal{L}_{1}^{3}$ (the exceptional case, characterized by the fact that the symmetry generators are functionally linearly dependent $[14,15,16$, 17, 18, 19, 22]),

10. $E 16: V=\frac{1}{\sqrt{x^{2}+y^{2}}}\left(\alpha+\frac{\beta}{y+\sqrt{x^{2}+y^{2}}}+\frac{\gamma}{y-\sqrt{x^{2}+y^{2}}}\right), \mathcal{R}^{2}=\mathcal{L}_{1}\left(\mathcal{L}_{1} \mathcal{H}+\mathcal{L}_{2}^{2}\right)$,

11. $E 17: V=\frac{\alpha}{\sqrt{x^{2}+y^{2}}}+\frac{\beta}{(x+i y)^{2}}+\frac{\gamma}{(x+i y) \sqrt{x^{2}+y^{2}}}, \mathcal{R}^{2}=\mathcal{L}_{1} \mathcal{L}_{2}^{2}$, 
12. $E 19: V=\frac{\alpha(x+i y)}{\sqrt{(x+i y)^{2}-4}}+\frac{\beta}{\sqrt{(x-i y)(x+i y+2)}}+\frac{\gamma}{\sqrt{(x-i y)(x+i y-2)}}, \mathcal{R}^{2}=\mathcal{L}_{1}\left(\mathcal{L}_{2}^{2}+\mathcal{H}^{2}\right)$,

13. $E 20: V=\frac{1}{\sqrt{x^{2}+y^{2}}}\left(\alpha+\beta \sqrt{x+\sqrt{x^{2}+y^{2}}}+\gamma \sqrt{x-\sqrt{x^{2}+y^{2}}}\right), \mathcal{R}^{2}=\mathcal{H}\left(\mathcal{L}_{1}^{2}+\mathcal{L}_{2}^{2}\right)$.

Systems on the complex 2-sphere: $\mathcal{H} \equiv \mathcal{J}_{23}^{2}+\mathcal{J}_{13}^{2}+\mathcal{J}_{12}^{2}+V=E$. Here, $\mathcal{J}_{k \ell}=s_{k} p_{s_{\ell}}-s_{\ell} p_{s_{k}}$ and $s_{1}^{2}+s_{2}^{2}+s_{3}^{2}=1$.

1. $S 1: V=\frac{\alpha}{\left(s_{1}+i s_{2}\right)^{2}}+\frac{\beta s_{3}}{\left(s_{1}+i s_{2}\right)^{2}}+\frac{\gamma\left(1-4 s_{3}^{2}\right)}{\left(s_{1}+i s_{2}\right)^{4}}, \mathcal{R}^{2}=\mathcal{L}_{1}^{3}$,

2. $S 2: V=\frac{\alpha}{s_{3}^{2}}+\frac{\beta}{\left(s_{1}+i s_{2}\right)^{2}}+\frac{\gamma\left(s_{1}-i s_{2}\right)}{\left(s_{1}+i s_{2}\right)^{3}}, \mathcal{R}^{2}=\mathcal{L}_{1} \mathcal{L}_{2}^{2}$,

3. $S 4: V=\frac{\alpha}{\left(s_{1}+i s_{2}\right)^{2}}+\frac{\beta s_{3}}{\sqrt{s_{1}^{2}+s_{2}^{2}}}+\frac{\gamma}{\left(s_{1}+i s_{2}\right) \sqrt{s_{1}^{2}+s_{2}^{2}}}, \mathcal{R}^{2}=\mathcal{L}_{1} \mathcal{L}_{2}^{2}$,

4. $S 7: V=\frac{\alpha s_{3}}{\sqrt{s_{1}^{2}+s_{2}^{2}}}+\frac{\beta s_{1}}{s_{2}^{2} \sqrt{s_{1}^{2}+s_{2}^{2}}}+\frac{\gamma}{s_{2}^{2}}, \mathcal{R}^{2}=\mathcal{L}_{1}^{2} \mathcal{L}_{2}+\mathcal{L}_{2}^{2} \mathcal{L}_{1}-\frac{1}{16} \mathcal{L}_{1}^{2} \mathcal{H}$,

5. $S 8: V=\frac{\alpha s_{2}}{\sqrt{s_{1}^{2}+s_{3}^{2}}}+\frac{\beta\left(s_{2}+i s_{1}+s_{3}\right)}{\sqrt{\left(s_{2}+i s_{1}\right)\left(s_{3}+i s_{1}\right)}}+\frac{\gamma\left(s_{2}+i s_{1}-s_{3}\right)}{\sqrt{\left(s_{2}+i s_{1}\right)\left(s_{3}-i s_{1}\right)}}, \mathcal{R}^{2}=\mathcal{L}_{1}^{2} \mathcal{L}_{2}+\mathcal{L}_{1} \mathcal{L}_{2}^{2}-\frac{1}{4} \mathcal{L}_{1} \mathcal{L}_{2} \mathcal{H}$,

6. $S 9: V=\frac{\alpha}{s_{1}^{2}}+\frac{\beta}{s_{2}^{2}}+\frac{\gamma}{s_{3}^{2}}, \mathcal{R}^{2}=\mathcal{L}_{1}^{2} \mathcal{L}_{2}+\mathcal{L}_{1} \mathcal{L}_{2}^{2}+\frac{1}{16} \mathcal{L}_{1} \mathcal{L}_{2} \mathcal{H}$.

Darboux 1 systems: $\mathcal{H} \equiv \frac{1}{4 x}\left(p_{x}^{2}+p_{y}^{2}\right)+V=E$.

1. $D 1 A: V=\frac{b_{1}(2 x-2 b+i y)}{x \sqrt{x-b+i y}}+\frac{b_{2}}{x \sqrt{x-b+i y}}+\frac{b_{3}}{x}+b_{4}, \mathcal{R}^{2}=\mathcal{L}_{1}^{3}+\mathcal{L}_{2} \mathcal{L}_{1} \mathcal{H}-b \mathcal{L}_{1}^{2} \mathcal{H}-2 i b \mathcal{H}^{2} \mathcal{L}_{2}$,

2. $D 1 B: V=\frac{b_{1}\left(4 x^{2}+y^{2}\right)}{x}+\frac{b_{2}}{x}+\frac{b_{3}}{x y^{2}}+b_{4}, \mathcal{R}^{2}=\mathcal{L}_{1}^{3}+\mathcal{L}_{2} \mathcal{L}_{1} \mathcal{H}$,

3. $D 1 C V=\frac{b_{1}\left(x^{2}+y^{2}\right)}{x}+\frac{b_{2}}{x}+\frac{b_{3} y}{x}+b_{4}, \mathcal{R}^{2}=\mathcal{L}_{2} \mathcal{H}^{2}$.

Darboux 2 systems: $\mathcal{H} \equiv \frac{x^{2}}{x^{2}+1}\left(p_{x}^{2}+p_{y}^{2}\right)+V=E$.

1. D2A: $V=\frac{x^{2}}{x^{2}+1}\left(b_{1}\left(x^{2}+4 y^{2}\right)+\frac{b_{2}}{x^{2}}+b_{3} y\right)+b_{4}, \mathcal{R}^{2}=\mathcal{L}_{1}^{3}+\mathcal{L}_{1}^{2} \mathcal{H}+\frac{1}{4} \mathcal{L}_{1} \mathcal{H}^{2}$,

2. D2B: $V=\frac{x^{2}}{x^{2}+1}\left(b_{1}\left(x^{2}+y^{2}\right)+\frac{b_{2}}{x^{2}}+\frac{b_{3}}{y^{2}}\right)+b_{4}, \mathcal{R}^{2}=\mathcal{L}_{1} \mathcal{L}_{2}^{2}+\mathcal{L}_{1} \mathcal{L}_{2} \mathcal{H}-\frac{1}{16} \mathcal{L}_{2} \mathcal{H}^{2}$,

3. $D 2 C: V=\frac{x^{2}}{\sqrt{x^{2}+y^{2}}\left(x^{2}+1\right)}\left(b_{1}+\frac{b_{2}}{y+\sqrt{x^{2}+y^{2}}}+\frac{b_{3}}{y-\sqrt{x^{2}+y^{2}}}\right)+b_{4}, \mathcal{R}^{2}=\mathcal{L}_{1} \mathcal{L}_{2}^{2}+\mathcal{L}_{1}^{2} \mathcal{H}-\frac{1}{4} \mathcal{L}_{1} \mathcal{H}^{2}$.

Darboux 3 systems: $\mathcal{H} \equiv \frac{1}{2} \frac{e^{2 x}}{e^{x}+1}\left(p_{x}^{2}+p_{y}^{2}\right)+V=E$.

1. D3A: $V=\frac{b_{1}}{1+e^{x}}+\frac{b_{2} e^{x}}{\sqrt{1+2 e^{x+i y}}\left(1+e^{x}\right)}+\frac{b_{3} e^{x+i y}}{\sqrt{1+2 e^{x+i y}}\left(1+e^{x}\right)}+b_{4}, \mathcal{R}^{2}=\mathcal{H}\left(\mathcal{L}_{1}^{2}+\mathcal{L}_{2}^{2}-\mathcal{H}^{2}\right)$,

2. D3B: $V=\frac{e^{x}}{e^{x}+1}\left(b_{1}+e^{-\frac{x}{2}}\left(b_{2} \cos \frac{y}{2}+b_{3} \sin \frac{y}{2}\right)\right)+b_{4}, \mathcal{R}^{2}=\mathcal{L}_{1} \mathcal{L}_{2}^{2}+\mathcal{H} \mathcal{L}_{1}^{2}-\frac{1}{4} \mathcal{H}^{2} \mathcal{L}_{1}$,

3. D3C: $V=\frac{e^{x}}{e^{x}+1}\left(b_{1}+e^{x}\left(\frac{b_{2}}{\cos ^{2} \frac{y}{2}}+\frac{b_{3}}{\sin ^{2} \frac{y}{2}}\right)\right)+b_{4}, \mathcal{R}^{2}=\mathcal{L}_{1} \mathcal{L}_{2}^{2}+\mathcal{L}_{1}^{2} \mathcal{H}-\frac{1}{8} \mathcal{L}_{1} \mathcal{H}^{2}$,

4. D3D: $V=\frac{e^{2 x}}{1+e^{x}}\left(b_{1} e^{-i y}+b_{2} e^{-2 i y}\right)+\frac{b_{3}}{1+e^{x}}+b_{4}, \mathcal{R}^{2}=\mathcal{L}_{1} \mathcal{L}_{2}^{2}+\mathcal{L}_{1} \mathcal{L}_{2} \mathcal{H}+\mathcal{L}_{2} \mathcal{H}^{2}-\mathcal{H}^{3}$.

Darboux 4 systems: $\mathcal{H} \equiv-\frac{\sin ^{2} 2 x}{2 \cos 2 x+b}\left(p_{x}^{2}+p_{y}^{2}\right)+V=E$.

1. D4(b) $A: V=\frac{\sin ^{2} 2 x}{2 \cos 2 x+b}\left(\frac{b_{1}}{\sinh ^{2} y}+\frac{b_{2}}{\sinh ^{2} 2 y}\right)+\frac{b_{3}}{2 \cos 2 x+b}+b_{4}, \mathcal{R}^{2}=\mathcal{L}_{1} \mathcal{L}_{2}^{2}$,

2. $D 4(b) B: V=\frac{\sin ^{2} 2 x}{2 \cos 2 x+b}\left(\frac{b_{1}}{\sin ^{2} 2 x}+b_{2} e^{4 y}+b_{3} e^{2 y}\right)+b_{4}, \mathcal{R}^{2}=\mathcal{L}_{1} \mathcal{L}_{2}^{2}+\mathcal{L}_{1}^{2} \mathcal{L}_{2}+b \mathcal{H} \mathcal{L}_{2}^{2}-4 \mathcal{H}^{2} \mathcal{L}_{2}$,

3. $D 4(b) C: V=\frac{e^{2 y}}{\frac{b+2}{\sin ^{2} x}+\frac{b-2}{\cos ^{2} x}}\left(\frac{b_{1}}{Z+\left(1-e^{2 y}\right) \sqrt{Z}}+\frac{b_{2}}{Z+\left(1+e^{2 y}\right) \sqrt{Z}}+\frac{b_{3} e^{-2 y}}{\cos ^{2} x}\right)+b_{4}$, $\mathcal{R}^{2}=-\frac{b}{16^{3}} \mathcal{H}^{3}+\mathcal{L}_{1}^{2} \mathcal{L}_{2}+\mathcal{L}_{1} \mathcal{L}_{2}^{2}-\frac{b}{16} \mathcal{L}_{1} \mathcal{L}_{2} \mathcal{H}-\frac{b}{16} \mathcal{L}_{2}^{2} \mathcal{H}+\frac{1}{256} \mathcal{L}_{1} \mathcal{H}^{2}$. 
Note: Systems $D 4(b) A, D 4(b) B, D 4(b) C$ are in fact families of distinct systems parametrized by $b$, and $E 15$ is a family of systems parametrized by the function $f$. The parameters $b$ can be normalized away in systems $E 7, D 1 A$, but it is convenient to keep them.

Generic Koenigs spaces: (We do not list the relatively unenlightening expressions of $\mathcal{R}^{2}$ for the Koenigs spaces. Each involves 4 arbitrary parameters obtained via a generic Stäckel transformation from a constant curvature system.)

1. $K[1,1,1,1]: \mathcal{H} \equiv \frac{1}{V\left(b_{1}, b_{2}, b_{3}, b_{4}\right)}\left(p_{x}^{2}+p_{y}^{2}+V\left(a_{1}, a_{2}, a_{3}, a_{4}\right)\right)=E$, $V\left(a_{1}, a_{2}, a_{3}, a_{4}\right)=\frac{a_{1}}{x^{2}}+\frac{a_{2}}{y^{2}}+\frac{4 a_{3}}{\left(x^{2}+y^{2}-1\right)^{2}}-\frac{4 a_{4}}{\left(x^{2}+y^{2}+1\right)^{2}}$,

2. $K[2,1,1]: \mathcal{H} \equiv \frac{1}{V\left(b_{1}, b_{2}, b_{3}, b_{4}\right)}\left(p_{x}^{2}+p_{y}^{2}+V\left(a_{1}, a_{2}, a_{3}, a_{4}\right)\right)=E$, $V\left(a_{1}, a_{2}, a_{3}, a_{4}\right)=\frac{a_{1}}{x^{2}}+\frac{a_{2}}{y^{2}}-a_{3}\left(x^{2}+y^{2}\right)+a_{4}$,

3. $K[2,2]: \mathcal{H} \equiv \frac{1}{V\left(b_{1}, b_{2}, b_{3}, b_{4}\right)}\left(p_{x}^{2}+p_{y}^{2}+V\left(a_{1}, a_{2}, a_{3}, a_{4}\right)\right)=E$, $V\left(a_{1}, a_{2}, a_{3}, a_{4}\right)=\frac{a_{1}}{(x+i y)^{2}}+\frac{a_{2}(x-i y)}{(x+i y)^{3}}+a_{3}-a_{4}\left(x^{2}+y^{2}\right)$,

4. $K[3,1]: \mathcal{H} \equiv \frac{1}{V\left(b_{1}, b_{2}, b_{3}, b_{4}\right)}\left(p_{x}^{2}+p_{y}^{2}+V\left(a_{1}, a_{2}, a_{3}, a_{4}\right)\right)=E$, $V\left(a_{1}, a_{2}, a_{3}, a_{4}\right)=a_{1}-a_{2} x+a_{3}\left(4 x^{2}+y^{2}\right)+\frac{a_{4}}{y^{2}}$,

5. $K[4]: \mathcal{H} \equiv=\frac{1}{V\left(b_{1}, b_{2}, b_{3}, b_{4}\right)}\left(p_{x}^{2}+p_{y}^{2}+V\left(a_{1}, a_{2}, a_{3}, a_{4}\right)\right)=E$, $V\left(a_{1}, a_{2}, a_{3}, a_{4}\right)=a_{1}-a_{2}(x+i y)+a_{3}\left(3(x+i y)^{2}+2(x-i y)\right)-a_{4}\left(4\left(x^{2}+y^{2}\right)+2(x+i y)^{3}\right)$,

6. $K[0]: \mathcal{H} \equiv=\frac{1}{V\left(b_{1}, b_{2}, b_{3}, b_{4}\right)}\left(p_{x}^{2}+p_{y}^{2}+V\left(a_{1}, a_{2}, a_{3}, a_{4}\right)\right)=E$, $V\left(a_{1}, a_{2}, a_{3}, a_{4}\right)=a_{1}-\left(a_{2} x+a_{3} y\right)+a_{4}\left(x^{2}+y^{2}\right)$.

\subsection{Contractions}

In [23] it has been shown that all the 2nd order superintegrable systems are obtained by taking coordinate limits of the generic system $S_{9}$ [22], or are obtained from these limits by a Stäckel transform (an invertible structure preserving mapping of superintegrable systems [14, 15, 16, 17, 18, 19]). Analogously all quadratic symmetry algebras of these systems are limits of that of $S_{9}$. These coordinate limits induce limit relations between the special functions associated as eigenfunctions of the quantum superintegrable systems. The limits also induce contractions of the associated quadratic algebras, and via the models of the irreducible representations of these algebras, limit relations between the associated special functions. The Askey scheme for orthogonal functions of hypergeometric type is an example of this [25]. For constant curvature systems the required limits are all induced by Inönü-Wigner-type Lie algebra contractions of $\mathfrak{o}(3, \mathbb{C})$ and $\mathfrak{e}(2, \mathbb{C})[11,31,37]$. Inönü-Wigner-type Lie algebra contractions have long been applied to relate separable coordinate systems and their associated special functions, see, e.g., [12, 13] for some more recent examples, but the application to quadratic algebras is due to the authors and their collaborators.

Recall the definition of (natural) Lie algebra contractions: Let $\left(A ;[;]_{A}\right),\left(B ;[;]_{B}\right)$ be two complex Lie algebras. We say that $B$ is a contraction of $A$ if for every $\epsilon \in(0,1]$ there exists a linear invertible map $t_{\epsilon}: B \rightarrow A$ such that for every $X, Y \in B, \lim _{\epsilon \rightarrow 0} t_{\epsilon}^{-1}\left[t_{\epsilon} X, t_{\epsilon} Y\right]_{A}=[X, Y]_{B}$. Thus, as $\epsilon \rightarrow 0$ the 1-parameter family of basis transformations can become singular but the structure constants of the Lie algebra go to a finite limit, necessarily that of another Lie algebra. The contractions of the symmetry algebras of $2 \mathrm{D}$ constant curvature spaces have long since been classified [23]. There are 6 nontrivial contractions of $\mathfrak{e}(2, \mathbb{C})$ and 4 of $\mathfrak{o}(3, \mathbb{C})$. They are each induced by coordinate limits. Just as for Lie algebras we can define a contraction of a quadratic algebra in terms of 1-parameter families of basis changes in the algebra. As $\epsilon \rightarrow 0$ the 1parameter family of basis transformations becomes singular but the structure constants go to a finite limit [23]. 
Theorem 1.3. Every Lie algebra contraction of $A=\mathfrak{e}(2, \mathbb{C})$ or $A=\mathfrak{o}(3, \mathbb{C})$ induces a contraction of a free (zero potential) quadratic algebra $\tilde{Q}$ based on $A$, which in turn induces a contraction of the quadratic algebra $Q$ with potential. This is true for both classical and quantum algebras.

Similarly the coordinate limit associated with each contraction takes $H$ to a new superintegrable system with the contracted quadratic algebra. This relationship between coordinate limits, Lie algebra contractions and quadratic algebra contractions for superintegrable systems on constant curvature spaces breaks down for Darboux and Koenigs spaces. For Darboux spaces the Lie symmetry algebra is only 1-dimensional, and there is no Lie symmetry algebra at all for Koenigs spaces. Furthermore, there is the issue of finding a more systematic way of classifying the 44 distinct Helmholtz superintegrable systems on different manifolds, and their relations. These issues can be clarified by considering the Helmholtz systems as Laplace equations (with potential) on flat space. As announced in [27], the proper object to study is the conformal symmetry algebra $\mathfrak{s o}(4, \mathbb{C})$ of the flat space Laplacian and its contractions. The basic idea is that families of (Stäckel-equivalent) Helmholtz superintegrable systems on a variety of manifolds correspond to a single conformally superintegrable Laplace equation on flat space. We exploit this here in the case $n=2$, but it generalizes easily to all dimensions $n \geq 2$. The conformal symmetry algebra for Laplace equations with constant potential on flat space is the conformal algebra $\mathfrak{s o}(n+2, \mathbb{C})$.

In his 1894 thesis [1] Bôcher introduced a limit procedure based on the roots of quadratic forms to find families of $R$-separable solutions of the ordinary (zero potential) flat space Laplace equation in $n$ dimensions. An important feature of his work was the introduction of special projective coordinates in which the action of the conformal group $\mathfrak{s o}(n+2, \mathbb{C})$ on solutions of the Laplace equation can be linearized. For $n=2$ these are tetraspherical coordinates. In Sections 3 and 4 we describe in detail the Laplace equation mechanism and how it can be applied to systematize the classification of Helmholtz superintegrable systems and their relations via limits. We show that Bôcher's limit procedure can be interpreted as constructing generalized Inönü-Wigner Lie algebra contractions of $\mathfrak{s o}(4, \mathbb{C})$ to itself. We call these Bôcher contractions and show that they induce contractions of the conformal quadratic algebras associated with Laplace superintegrable systems. All of the limits of the Helmholtz systems classified before for $n=2[10,23]$ are induced by the larger class of Bôcher contractions [27]. In this paper we replace Bôcher's prescription by a precise definition of Bôcher contractions and introduce special Bôcher contractions, which are simpler and more easily classified.

\section{$22 \mathrm{D}$ conformal superintegrability of the 2 nd order}

Classical $\mathrm{nD}$ systems of Laplace type are of the form

$$
\mathcal{H} \equiv \sum_{i, j=1}^{n} g^{i j} p_{i} p_{j}+V=0 .
$$

A conformal symmetry of this equation is a function $\mathcal{S}(\mathbf{x}, \mathbf{p})$ in the variables $\mathbf{x}=\left(x_{1}, \ldots, x_{n}\right)$, polynomial in the momenta $\mathbf{p}=\left(p_{1}, \ldots, p_{n}\right)$, such that $\{\mathcal{S}, \mathcal{H}\}=\mathcal{R}_{\mathcal{S}} \mathcal{H}$ for some function $\mathcal{R}_{\mathcal{S}}(\mathbf{x}, \mathbf{p})$, polynomial in the momenta. Two conformal symmetries $\mathcal{S}, \mathcal{S}^{\prime}$ are identified if $\mathcal{S}=$ $\mathcal{S}^{\prime}+\mathcal{R H}$ for some function $\mathcal{R}(\mathbf{x}, \mathbf{p})$, polynomial in the momenta. (For short we will say that $\mathcal{S}=\mathcal{S}^{\prime}, \bmod \mathcal{H}$ and that $\mathcal{S}$ is a conformal constant of the motion (or conformal symmetry) if $\{\mathcal{S}, \mathcal{H}\}=0, \bmod (\mathcal{H})$.$) The system is conformally superintegrable for n>2$ if there are $2 n-1$ functionally independent conformal symmetries, $\mathcal{S}_{1}, \ldots, \mathcal{S}_{2 n-1}$ with $\mathcal{S}_{1}=\mathcal{H}$. It is second order conformally superintegrable if each symmetry $\mathcal{S}_{i}$ can be chosen to be a polynomial of at most second order in the momenta. There are obvious operator counterparts to these definitions for the operator Laplace equation $H \Psi \equiv\left(\Delta_{n}+V\right) \psi=0$. 
For $n=2$ the definition must be restricted, since for a potential $V=0$ there will be an infinite-dimensional space of conformal symmetries. We assume $V \neq 0$, possibly a constant.

Every $2 D$ Riemannian manifold is conformally flat, so we can always find a Cartesian-like coordinate system with coordinates $\mathbf{x} \equiv(x, y) \equiv\left(x_{1}, x_{2}\right)$ such that the Laplace equation takes the form

$$
\tilde{\mathcal{H}}=\frac{1}{\lambda(x, y)}\left(p_{x}^{2}+p_{y}^{2}\right)+\tilde{V}(\mathbf{x})=0
$$

However, this equation is equivalent to the flat space equation

$$
\mathcal{H} \equiv p_{x}^{2}+p_{y}^{2}+V(\mathbf{x})=0, \quad V(\mathbf{x})=\lambda(\mathbf{x}) \tilde{V}(\mathbf{x}) .
$$

In particular, the conformal symmetries of (2.1) are identical with the conformal symmetries of (2.2). Thus without loss of generality we can assume the manifold is flat space with $\lambda \equiv 1$.

In general the space of 2 nd order conformal symmetries could be infinite-dimensional. However, the requirement that $H$ have a multiparameter potential reduces the possible symmetries to a finite-dimensional space. The result, from the Bertrand-Darboux conditions, is that the pure 2nd order polynomial terms in conformal symmetries belong to the space spanned by symmetrized products of the conformal Killing vectors

$$
\begin{aligned}
& P_{1}=p_{x}, \quad P_{2}=p_{y}, \quad J=x p_{y}-y p_{x}, \quad D=x p_{x}+y p_{y}, \\
& K_{1}=\left(x^{2}-y^{2}\right) p_{x}+2 x y p_{y}, \quad K_{2}=\left(y^{2}-x^{2}\right) p_{y}+2 x y p_{x} .
\end{aligned}
$$

For a given multiparameter potential only a subspace of these conformal tensors occurs.

\subsection{The conformal Stäckel transform}

We review briefly the concept of the conformal Stäckel transform [24]. Suppose we have a second order conformal superintegrable system

$$
\mathcal{H} \equiv \frac{1}{\lambda(x, y)}\left(p_{x}^{2}+p_{y}^{2}\right)+V(x, y)=0, \quad \mathcal{H} \equiv \mathcal{H}_{0}+V
$$

with $V$ the general potential solution for this system, and suppose $U(x, y)$ is a particular potential solution, nonzero in an open set. The conformal Stäckel transform induced by $U$ is the system

$$
\tilde{\mathcal{H}}=E, \quad \tilde{\mathcal{H}} \equiv \frac{1}{\tilde{\lambda}}\left(p_{x}^{2}+p_{y}^{2}\right)+\tilde{V}
$$

where $\tilde{\lambda}=\lambda U, \tilde{V}=\frac{V}{U}$. In $[20,27]$ we proved

Theorem 2.1. The transformed (Helmholtz) system $\tilde{\mathcal{H}}$ is superintegrable (in the nonconformal sense).

This result shows that any second order conformal Laplace superintegrable system admitting a nonconstant potential $U$ can be Stäckel transformed to a Helmholtz superintegrable system. This operation is invertible, but the inverse is not a Stäckel transform. By choosing all possible special potentials $U$ associated with the fixed Laplace system (2.4) we generate the equivalence class of all Helmholtz superintegrable systems (2.5) obtainable through this process. As is easy to check, any two Helmholtz superintegrable systems lie in the same equivalence class if and only if they are Stäckel equivalent in the standard sense, see [27, Theorem 4]. All Helmholtz superintegrable systems are related to conformal Laplace systems in this way, so the study of all Helmholtz superintegrability on conformally flat manifolds can be reduced to the study of all 
conformal Laplace superintegrable systems on flat space. All of these results have direct analogs for operator Laplace systems.

The basic structure of quadratic algebras for nondegenerate Helmholtz superintegrable systems is preserved under the transformation to Laplace equations, except that all identities hold $\bmod \mathcal{H}$ :

Theorem 2.2 ([27]). The symmetries $\mathcal{S}_{1}, \mathcal{S}_{2}$ of the $2 D$ nondegenerate conformal superintegrable Hamiltonian $\mathcal{H}$ generate a quadratic algebra

$$
\begin{aligned}
& \left\{\mathcal{R}, \mathcal{S}_{1}\right\}=f^{(1)}\left(\mathcal{S}_{1}, \mathcal{S}_{2}, \alpha_{1}, \alpha_{2}, \alpha_{3}, \alpha_{4}\right), \quad\left\{\mathcal{R}, \mathcal{S}_{2}\right\}=f^{(2)}\left(\mathcal{S}_{1}, \mathcal{S}_{2}, \alpha_{1}, \alpha_{2}, \alpha_{3}, \alpha_{4}\right), \\
& \mathcal{R}^{2}=f^{(3)}\left(\mathcal{S}_{1}, \mathcal{S}_{2}, \alpha_{1}, \alpha_{2}, \alpha_{3}, \alpha_{4}\right),
\end{aligned}
$$

where $\mathcal{R}=\left\{\mathcal{S}_{1}, \mathcal{S}_{2}\right\}$ and all identities hold $\bmod \mathcal{H}$. Here the $\alpha_{j}$ are the parameters in the nondegenerate potential.

A crucial observation now is that the free parts (those parts that one obtains by setting all the $a_{i}$ to zero) of the generators for 2 nd order conformal superintegrable systems lie in the universal enveloping algebra of the conformal Lie algebra, mod $\mathcal{H}$. Thus for the 2D case it follows that contractions of $\mathfrak{s o}(4, \mathbb{C})$ induce contractions of the conformal quadratic algebras of 2 nd order superintegrable systems with nondegenerate potentials, and contractions of one system into another. In [27] it is shown how these Laplace contractions then induce contractions of Helmholtz superintegrable systems.

\section{Tetraspherical coordinates and Laplace systems}

As already mentioned, the free parts of the 2nd order conformal symmetries of the Laplace equation $\mathcal{H} \equiv p_{x}^{2}+p_{y}^{2}+V(\mathbf{x})=0$ lie in the universal enveloping algebra of $\mathfrak{s o}(4, \mathbb{C})$ with generators $(2.3)$. To linearize the action of these $\mathfrak{s o}(n+2, \mathbb{C})$ operators on Laplace equations in $n$ dimensions, Bôcher introduced a family of projective coordinates on the null cone in $n+2$ dimensions. In our case $n=2$ these are the tetraspherical coordinates $\left(x_{1}, \ldots, x_{4}\right)$. They satisfy

$x_{1}^{2}+x_{2}^{2}+x_{3}^{2}+x_{4}^{2}=0$ (the null cone) and $\sum_{k=1}^{4} x_{k} \partial_{x_{k}}=0$. They are projective coordinates on the null cone and have 2 degrees of freedom. Their principal advantage over flat space Cartesian coordinates is that the action of the conformal algebra $(2.3)$ and of the conformal group $\mathrm{SO}(4, \mathbb{C})$ is linearized in tetraspherical coordinates.

\subsection{Relation to Cartesian coordinates $(x, y)$ and coordinates on the 2-sphere $\left(s_{1}, s_{2}, s_{3}\right)$}

$$
\begin{aligned}
& x_{1}=2 X T, \quad x_{2}=2 Y T, \quad x_{3}=X^{2}+Y^{2}-T^{2}, \quad x_{4}=i\left(X^{2}+Y^{2}+T^{2}\right), \\
& x=\frac{X}{T}=-\frac{x_{1}}{x_{3}+i x_{4}}, \quad y=\frac{Y}{T}=-\frac{x_{2}}{x_{3}+i x_{4}}, \quad x=\frac{s_{1}}{1+s_{3}}, \quad y=\frac{s_{2}}{1+s_{3}} . \quad
\end{aligned}
$$

The projective variables $X, Y, T$ are defined by these relations

$$
\begin{aligned}
& s_{1}=\frac{2 x}{x^{2}+y^{2}+1}, \quad s_{2}=\frac{2 y}{x^{2}+y^{2}+1}, \quad s_{3}=\frac{1-x^{2}-y^{2}}{x^{2}+y^{2}+1}, \\
& \mathcal{H} \equiv p_{x}^{2}+p_{y}^{2}+\tilde{V}=\left(x_{3}+i x_{4}\right)^{2}\left(\sum_{k=1}^{4} p_{x_{k}}^{2}+V\right)=\left(1+s_{3}\right)^{2}\left(\sum_{j=1}^{3} p_{s_{j}}^{2}+V\right), \\
& \tilde{V}=\left(x_{3}+i x_{4}\right)^{2} V, \quad\left(1+s_{3}\right)=-i \frac{\left(x_{3}+i x_{4}\right)}{x_{4}}, \quad s_{1}=\frac{i x_{1}}{x_{4}}, \quad s_{2}=\frac{i x_{2}}{x_{4}}, \quad s_{3}=-\frac{i x_{3}}{x_{4}} .
\end{aligned}
$$


Thus the Laplace equation $\mathcal{H} \equiv p_{x}^{2}+p_{y}^{2}+\tilde{V}$ in Cartesian coordinates becomes $\sum_{k=1}^{4} p_{x_{k}}^{2}+V=0$ in tetraspherical coordinates.

\subsection{Relation to flat space and 2-sphere 1st order conformal constants of the motion}

We define

$$
L_{j k}=x_{j} \partial_{x_{k}}-x_{k} \partial_{x_{j}}, \quad 1 \leq j, k \leq 4, \quad j \neq k,
$$

where $L_{j k}=-L_{k j}$. The generators for flat space conformal symmetries (2.3) are related to these via

$$
\begin{aligned}
& P_{1}=L_{13}+i L_{14}, \quad P_{2}=L_{23}+i L_{24}, \quad D=i L_{34}, \quad J=L_{12}, \\
& K_{j}=L_{j 3}-i L_{j 4}, \quad j=1,2 .
\end{aligned}
$$

The generators for 2-sphere conformal symmetries are related to the $L_{j k}$ via

$$
\begin{aligned}
& L_{12}=J_{12}=s_{1} p_{s_{2}}-s_{2} p_{s_{1}}, \quad L_{31}=J_{31}, \quad L_{23}=J_{23}, \\
& L_{j 4}=-i p_{s_{j}}, \quad j=1,2,3 .
\end{aligned}
$$

In identifying tetraspherical coordinates we can always permute the parameters $1, \ldots, 4$. Also, we can apply an arbitrary $\mathrm{SO}(4, \mathbb{C})$ transformation to the tetraspherical coordinates, so the above relations between Euclidean and tetraspherical coordinates are far from being unique.

\subsection{The 6 Laplace superintegrable systems with nondegenerate potentials}

The systems are all of the form

$$
\left(\sum_{j=1}^{4} \partial_{x_{j}}^{2}+V(\mathbf{x})\right) \Psi=0
$$

in tetraspherical coordinates, or $\left(\partial_{x}^{2}+\partial_{y}^{2}+\tilde{V}\right) \Psi=0$ as a flat space system in Cartesian coordinates. Each Laplace system is an equivalence class of Stäckel equivalent Helmholtz systems. In each case the expression for $\mathcal{R}^{2}$ in the conformal symmetry algebra can be put in a normal form which is a polynomial in $\mathcal{L}_{j}, a_{k}$ of order $\leq 3$. We show the terms of order $\geq 2$ in the $\mathcal{L}_{j}$ alone. The parameter $\alpha$ is linear in the $a_{j}$. The remaining terms are of lower order in the $\mathcal{L}_{j}$ : LOT. The potentials are

$$
\begin{aligned}
& V_{[1,1,1,1]}=\frac{a_{1}}{x_{1}^{2}}+\frac{a_{2}}{x_{2}^{2}}+\frac{a_{3}}{x_{3}^{2}}+\frac{a_{4}}{x_{4}^{2}}, \\
& \tilde{V}_{[1,1,1,1]}=\frac{a_{1}}{x^{2}}+\frac{a_{2}}{y^{2}}+\frac{4 a_{3}}{\left(x^{2}+y^{2}-1\right)^{2}}-\frac{4 a_{4}}{\left(x^{2}+y^{2}+1\right)^{2}}, \\
& \mathcal{R}^{2}=\mathcal{L}_{1} \mathcal{L}_{2}\left(\mathcal{L}_{1}+\mathcal{L}_{2}\right)+\alpha \mathcal{L}_{1} \mathcal{L}_{2}+\text { LOT. }
\end{aligned}
$$

Stäckel equivalent systems: $S 9, S 8, S 7, D 4 B(b), D 4 C(b), K[1,1,1,1]$.

$$
\begin{aligned}
& V_{[2,1,1]}=\frac{a_{1}}{x_{1}^{2}}+\frac{a_{2}}{x_{2}^{2}}+\frac{a_{3}\left(x_{3}-i x_{4}\right)}{\left(x_{3}+i x_{4}\right)^{3}}+\frac{a_{4}}{\left(x_{3}+i x_{4}\right)^{2}}, \\
& \tilde{V}_{[2,1,1]}=\frac{a_{1}}{x^{2}}+\frac{a_{2}}{y^{2}}-a_{3}\left(x^{2}+y^{2}\right)+a_{4}, \\
& \mathcal{R}^{2}=\mathcal{L}_{1}^{2} \mathcal{L}_{2}+\alpha \mathcal{L}_{2}^{2}+\text { LOT. }
\end{aligned}
$$


Stäckel equivalent systems: $S 4, S 2, E 1, E 16, D 4 A(b), D 3 B, D 2 B, D 2 C, K[2,1,1]$.

$$
\begin{aligned}
& V_{[2,2]}=\frac{a_{1}}{\left(x_{1}+i x_{2}\right)^{2}}+\frac{a_{2}\left(x_{1}-i x_{2}\right)}{\left(x_{1}+i x_{2}\right)^{3}}+\frac{a_{3}}{\left(x_{3}+i x_{4}\right)^{2}}+\frac{a_{4}\left(x_{3}-i x_{4}\right)}{\left(x_{3}+i x_{4}\right)^{3}}, \\
& \tilde{V}_{[2,2]}=\frac{a_{1}}{(x+i y)^{2}}+\frac{a_{2}(x-i y)}{(x+i y)^{3}}+a_{3}-a_{4}\left(x^{2}+y^{2}\right), \\
& \mathcal{R}^{2}=\mathcal{L}_{1}^{2} \mathcal{L}_{2}+\text { LOT. }
\end{aligned}
$$

Stäckel equivalent systems: $E 8, E 17, E 7, E 19, D 3 C, D 3 D, K[2,2]$.

$$
\begin{aligned}
& V_{[3,1]}=\frac{a_{1}}{\left(x_{3}+i x_{4}\right)^{2}}+\frac{a_{2} x_{1}}{\left(x_{3}+i x_{4}\right)^{3}}+\frac{a_{3}\left(4 x_{1}^{2}+x_{2}^{2}\right)}{\left(x_{3}+i x_{4}\right)^{4}}+\frac{a_{4}}{x_{2}{ }^{2}}, \\
& \tilde{V}_{[3,1]}=a_{1}-a_{2} x+a_{3}\left(4 x^{2}+y^{2}\right)+\frac{a_{4}}{y^{2}}, \\
& \mathcal{R}^{2}=\mathcal{L}_{1}^{3}+\alpha \mathcal{L}_{2}^{2}+\text { LOT. }
\end{aligned}
$$

Stäckel equivalent systems: $S 1, E 2, D 1 B, D 2 A, K[3,1]$.

$$
\begin{aligned}
V_{[4]}= & \frac{a_{1}}{\left(x_{3}+i x_{4}\right)^{2}}+a_{2} \frac{x_{1}+i x_{2}}{\left(x_{3}+i x_{4}\right)^{3}}+a_{3} \frac{3\left(x_{1}+i x_{2}\right)^{2}-2\left(x_{3}+i x_{4}\right)\left(x_{1}-i x_{2}\right)}{\left(x_{3}+i x_{4}\right)^{4}} \\
& +a_{4} \frac{4\left(x_{3}+i x_{4}\right)\left(x_{3}^{2}+x_{4}^{2}\right)+2\left(x_{1}+i x_{2}\right)^{3}}{\left(x_{3}+i x_{4}\right)^{5}}, \\
\tilde{V}_{[4]}= & a_{1}-a_{2}(x+i y)+a_{3}\left(3(x+i y)^{2}+2(x-i y)\right)-a_{4}\left(4\left(x^{2}+y^{2}\right)+2(x+i y)^{3}\right), \\
\mathcal{R}^{2}= & \mathcal{L}_{1}^{3}+\alpha \mathcal{L}_{1} \mathcal{L}_{2}+\mathrm{LOT} .
\end{aligned}
$$

Stäckel equivalent systems: $E 10, E 9, D 1 A, K[4]$.

$$
\begin{aligned}
& V_{[0]}=\frac{a_{1}}{\left(x_{3}+i x_{4}\right)^{2}}+\frac{a_{2} x_{1}+a_{3} x_{2}}{\left(x_{3}+i x_{4}\right)^{3}}+a_{4} \frac{x_{1}^{2}+x_{2}^{2}}{\left(x_{3}+i x_{4}\right)^{4}}, \\
& \tilde{V}_{[0]}=a_{1}-\left(a_{2} x+a_{3} y\right)+a_{4}\left(x^{2}+y^{2}\right), \\
& \mathcal{R}^{2}=\alpha \mathcal{L}_{1} \mathcal{L}_{2}+\text { LOT. }
\end{aligned}
$$

Stäckel equivalent systems: $E 20, E 11, E 3^{\prime}, D 1 C, D 3 A, K[0]$.

\section{Definition and composition of Bôcher contractions}

Before introducing precise definitions, let us note that all geometrical contractions of $\mathfrak{e}(2, \mathbb{C}) \rightarrow$ $\mathfrak{e}(2, \mathbb{C})$ and $\mathfrak{s o}(3, \mathbb{C}) \rightarrow \mathfrak{s o}(3, \mathbb{C}), \mathfrak{e}(2, \mathbb{C})$, i.e., pointwise coordinate limits of functions on flat space or the sphere as classified in [23], induce geometrical contractions of $\mathfrak{s o}(4, \mathbb{C}) \rightarrow \mathfrak{s o}(4, \mathbb{C})$. Recall that a basis for $\mathfrak{s o}(4, \mathbb{C})$ is $(2.3)$ where the subset $P_{1}, P_{2}, J$ forms a basis for $\mathfrak{e}(2, \mathbb{C})$. As an example, consider the coordinate limit $x=\epsilon x^{\prime}, y=\epsilon y^{\prime}$. This induces the contraction $\epsilon P_{1}=P_{1}^{\prime}$, $\epsilon P_{2}=P_{2}^{\prime}, J=J^{\prime}$ of $\mathfrak{e}(2, \mathbb{C})$ and, further, the contraction $D=D^{\prime}, K_{1}=\epsilon K_{1}^{\prime}, K_{2}=\epsilon K_{2}^{\prime}$ of $\mathfrak{s o}(4, \mathcal{C})$. The other contractions of $\mathfrak{e}(2, \mathbb{C})$ work similarly.

For $\mathfrak{s o}(3, \mathbb{C})$ we have the basis $J_{23}, J_{31}, J_{12}$, where

$$
s_{1}^{2}+s_{2}^{2}+s_{3}^{2}=1, \quad s_{1} p_{s_{1}}+s_{2} p_{s_{2}}+s_{3} p_{s_{3}}=0 .
$$

The generators for the conformal symmetry algebra of the $\mathfrak{s o}(3, \mathbb{C})$ Laplace equation are related to the $L_{j k}$ basis for $\mathfrak{s o}(4, \mathbb{C})$ via $(3.1)$. Now consider the example limit $s_{1}=\epsilon x^{\prime}, s_{2}=\epsilon y^{\prime}$. It induces the contraction

$$
\epsilon J_{23}=-p_{y^{\prime}}, \quad \epsilon J_{31}=p_{x^{\prime}}, \quad J_{12}=x^{\prime} p_{y^{\prime}}-y^{\prime} p_{x^{\prime}}
$$


of $\mathfrak{s o}(3, \mathbb{C})$ to $\mathfrak{e}(2, \mathbb{C})$ and the contraction

$$
\begin{aligned}
& L_{12}=x^{\prime} p_{y^{\prime}}-y^{\prime} p_{x^{\prime}}=J^{\prime}, \quad i \epsilon L_{14}=p_{x^{\prime}}=P_{1}^{\prime}, \quad i \epsilon L_{24}=p_{y^{\prime}}=P_{2}^{\prime}, \\
& -\frac{2}{\epsilon}\left(i L_{14}+L_{13}\right)=\left(x^{\prime 2}-y^{\prime 2}\right) p_{x^{\prime}}+2 x^{\prime} y^{\prime} p_{y^{\prime}}+O(\epsilon)=K_{1}^{\prime}+O(\epsilon), \\
& -\frac{2}{\epsilon}\left(i L_{24}+L_{23}\right)=\left(y^{\prime 2}-x^{\prime 2}\right) p_{y^{\prime}}+2 x^{\prime} y^{\prime} p_{x^{\prime}}+O(\epsilon)=K_{2}^{\prime}+O(\epsilon),
\end{aligned}
$$

of $\mathfrak{s o}(4, \mathbb{C})$ to itself. The other contractions of $\mathfrak{s o}(3, \mathbb{C})$ work similarly.

We now present a general definition of Bôcher contractions of $\mathfrak{s o}(4, \mathbb{C})$ to itself and demonstrate that the above induced contractions can be reformulated as Bôcher contractions. Let $\mathbf{x}=\mathbf{A}(\epsilon) \mathbf{y}$, and $\mathbf{x}=\left(x_{1}, \ldots, x_{4}\right), \mathbf{y}=\left(y_{1}, \ldots, y_{4}\right)$ be column vectors, and $\mathbf{A}=\left(A_{j k}(\epsilon)\right)$, be a $4 \times 4$ matrix with matrix elements

$$
A_{k j}(\epsilon)=\sum_{\ell=-N}^{N} a_{k j}^{\ell} \epsilon^{\ell}
$$

where $N$ is a nonnegative integer and the $a_{k j}^{\ell}$ are complex constants. (Here, $N$ can be arbitrarily large, but it must be finite in any particular case.) We say that the matrix $\mathbf{A}$ defines a Bôcher contraction of the conformal algebra $\mathfrak{s o}(4, \mathbb{C})$ to itself provided

$$
\begin{aligned}
& \text { 1) } \operatorname{det}(\mathbf{A})= \pm 1, \text { constant for all } \epsilon \neq 0, \\
& \text { 2) } \mathbf{x} \cdot \mathbf{x} \equiv \sum_{j=1}^{4} x_{i}(\epsilon)^{2}=\mathbf{y} \cdot \mathbf{y}+O(\epsilon)
\end{aligned}
$$

If, in addition, $\mathbf{A} \in O(4, \mathbb{C})$ for all $\epsilon \neq 0$ the matrix $\mathbf{A}$ defines a special Bôcher contraction. For a special Bôcher contraction $\mathbf{x} \cdot \mathbf{x}=\mathbf{y} \cdot \mathbf{y}$, with no error term.

We explain why this is a contraction in the generalized Inönü-Wigner sense. Let $L_{t s}=$ $x_{t} \partial_{x_{s}}-x_{s} \partial_{x_{t}}, s \neq t$ be a generator of $\mathfrak{s o}(4, \mathbb{C})$ and $\tilde{\mathbf{A}}(\epsilon)=\mathbf{A}^{-1}(\epsilon)$ be the matrix inverse. (Note that $\tilde{\mathbf{A}}$ also has an expansion of the form (4.1) in $\epsilon$.) We have the expansion

$$
L_{t s}=\sum_{k, \ell}\left(A_{t k} \tilde{A}_{\ell s}-A_{s k} \tilde{A}_{\ell t}\right) y_{k} \partial_{y_{\ell}}=\epsilon^{\alpha_{t s}}\left(\sum_{k \ell} F_{k \ell} y_{k} \partial_{y_{\ell}}+O(\epsilon)\right),
$$

where $\mathbf{F}$ is a constant nonzero matrix. Thus the integer $\alpha_{t s}$ is the smallest power of $\epsilon$ occurring in the expansion of $L_{t s}$. Now consider the product $L_{t s}(\mathbf{x} \cdot \mathbf{x})$. On one hand it is obvious that $L_{t s}(\mathbf{x} \cdot \mathbf{x}) \equiv 0$, but on the other hand the expansions (4.3), (4.4) yield

$$
L_{t s}(\mathbf{x} \cdot \mathbf{x})=\epsilon^{\alpha_{t s}}\left(\sum_{k \ell} F_{k \ell} y_{k} \partial_{y_{\ell}}\right)\left(\sum_{j} y_{j}^{2}\right)+O\left(\epsilon^{\alpha_{t s}}\right) .
$$

Thus, $\left(\sum_{k \ell} F_{k \ell} y_{k} \partial_{y_{\ell}}\right)\left(\sum_{j} y_{j}^{2}\right) \equiv 0$ for $\mathbf{F}$ a constant nonzero matrix. However, the only differential operators of the form $\sum_{k \ell} F_{k \ell} y_{k} \partial_{y_{\ell}}$ that map $\mathbf{y} \cdot \mathbf{y}$ to zero are elements of $\mathfrak{s o}(4, \mathbb{C})$ :

$$
\sum_{k \ell} F_{k \ell} y_{k} \partial_{y_{\ell}}=\sum_{j>k} b_{j k} L_{j k}^{\prime}, \quad L_{j k}^{\prime}=y_{j} \partial_{y_{k}}-y_{k} \partial_{y_{j}} .
$$

Thus

$$
\lim _{\epsilon \longrightarrow 0} \epsilon^{-\alpha_{t s}} L_{t s}=\sum_{j>k} b_{j k} L_{j k}^{\prime} \equiv L^{\prime}
$$


and this determines a limit of $L_{t s}$ to $L^{\prime}$. Similarly, if we apply this same procedure to the operator $L=\sum_{t>s} c(\epsilon)_{t s} L_{t s}$ for any rational polynomials $c_{t s}(\epsilon)$ we will obtain an operator $L^{\prime}=$ $\sum_{j>k} b_{j k} L_{j k}^{\prime}$ in the limit. Further, due to condition (4.2), by choosing the $c(\epsilon)_{t s}$ appropriately we can obtain any $L^{\prime} \in \mathfrak{s o}(4, \mathbb{C})$ in the limit. (Indeed, modulo rational functions of $\epsilon$, this is just the adjoint action of $O(4, \mathbb{C})$ on $\mathfrak{s o}(4, \mathbb{C})$. In this sense the mapping $L \rightarrow L^{\prime}$ is onto.)

Theorem 4.1. Suppose the matrix $\mathbf{A}(\epsilon)$ defines a Bôcher contraction of $\mathfrak{s o}(4, \mathbb{C})$. Let $\left\{L_{t_{i} s_{i}}\right.$, $i=1, \ldots, 6\}$ be an ordered linearly independent for $\mathfrak{s o}(4, \mathbb{C})$ such that $\alpha_{t_{1} s_{1}} \leq \alpha_{t_{2} s_{2}} \leq \cdots \leq \alpha_{t_{6} s_{6}}$. Then there is an ordered linearly independent set $\left\{L_{j}, j=1, \ldots, 6\right\}$ for $\mathfrak{s o}(4, \mathbb{C})$ such that

1) $L_{j} \in \operatorname{span}\left\{L_{t_{i} s_{i}}, i=1, \ldots, j\right\}$,

2) there are integers $\alpha_{1} \leq \alpha_{2} \leq \cdots \leq \alpha_{6}$ such that

$$
\lim _{\epsilon \rightarrow 0} \frac{L_{j}}{\epsilon^{\alpha_{j}}}=L_{j}^{\prime}, \quad 1 \leq j \leq 6,
$$

and $\left\{L_{j}^{\prime}, j=1, \ldots, 6\right\}$ forms a basis for $\mathfrak{s o}(4, \mathbb{C})$ in the $y_{k}$ variables.

Proof. The proof is by induction on $j$. For $j=1$ the result follows from (4.5). Assume the assertion is true for $j \leq j_{0}<6$. Then, due to the nonsingularity condition (4.2), we can always find polynomials in $\epsilon,\left\{a_{1}(\epsilon), a_{2}(\epsilon), \ldots, a_{j_{0}}(\epsilon)\right\}$ such that

$$
L_{j_{0}+1}=L_{t_{j_{0}+1}, s_{j_{0}+1}}-\sum_{i=1}^{j_{0}} a_{i} L_{i}=\epsilon^{\alpha_{j_{0}+1}} L_{j_{0}+1}^{\prime}+O\left(\epsilon^{\alpha_{j_{0}+2}}\right),
$$

where $L_{j_{0}+1}^{\prime}$ is linearly independent of $\left\{L_{i}^{\prime}, 1 \leq i \leq j_{0}\right\}$ and $\alpha_{j_{0}+1} \geq \alpha_{j_{0}}$.

In [27] we have used this theorem to compute explicitly the bases for the basic Bôcher contractions.

\subsection{Composition of Bôcher contractions}

Let $\mathbf{A}$ and $\mathbf{B}$ define Bôcher contractions of $\mathfrak{s o}(4, \mathbb{C})$ to itself. Thus there exist expansions

$$
\mathbf{x}\left(\epsilon_{1}\right) \cdot \mathbf{x}\left(\epsilon_{1}\right)=\mathbf{y} \cdot \mathbf{y}+O\left(\epsilon_{1}^{a}\right), \quad \mathbf{y}\left(\epsilon_{2}\right) \cdot \mathbf{y}\left(\epsilon_{2}\right)=\mathbf{z} \cdot \mathbf{z}+O\left(\epsilon_{2}^{b}\right)
$$

where

$$
\mathbf{x}=\mathbf{A}\left(\epsilon_{1}\right) \mathbf{y}, \quad \mathbf{y}\left(\epsilon_{2}\right)=\mathbf{B}\left(\epsilon_{2}\right) \mathbf{z} .
$$

Now let

$$
\mathbf{x}\left(\epsilon_{1}, \epsilon_{2}\right)=\mathbf{A}\left(\epsilon_{1}\right) \mathbf{y}\left(\epsilon_{2}\right)=\mathbf{A}\left(\epsilon_{1}\right) \mathbf{B}\left(\epsilon_{2}\right) \mathbf{z} .
$$

Then

$$
\mathbf{x}\left(\epsilon_{1}, \epsilon_{2}\right) \cdot \mathbf{x}\left(\epsilon_{1}, \epsilon_{2}\right)=\mathbf{y}\left(\epsilon_{2}\right) \cdot \mathbf{y}\left(\epsilon_{2}\right)+O_{\epsilon_{2}}\left(\epsilon_{1}^{a}\right)=\mathbf{z} \cdot \mathbf{z}+O\left(\epsilon_{2}^{b}\right)+\epsilon_{1}^{a} f\left(\epsilon_{1}, \epsilon_{2}, \mathbf{y}\right) .
$$

Now set $\epsilon_{1}=\epsilon^{m}, \epsilon_{2}=\epsilon$. It follows from these expansions that we can always find an $m>0$ such that

$$
\mathbf{x}\left(\epsilon^{m}, \epsilon\right) \cdot \mathbf{x}\left(\epsilon^{m}, \epsilon\right)=\mathbf{z} \cdot \mathbf{z}+O\left(\epsilon^{q}\right)
$$

and

$$
\lim _{\epsilon \rightarrow 0} \epsilon^{-\alpha_{t s}} L_{t s}=\sum_{j>k} c_{j k} L_{j k}^{\prime \prime} \equiv L^{\prime \prime}
$$

for some $q>0$, with $L^{\prime \prime}$ in the $\mathfrak{s o}(4, \mathbb{C})$ Lie algebra of operators such that $L^{\prime \prime}(\mathbf{z} \cdot \mathbf{z})=0$. Thus this composition of the $A$ and $B$ contractions yields a new Bôcher contraction. For special Bôcher contractions the composition is defined without restriction and the resulting contraction is uniquely determined for $\epsilon_{1}, \epsilon_{2}$ going to 0 independently. However, if we set $\epsilon_{2}=\epsilon_{1}^{m}$, in general the resulting contraction will depend on $m$. 


\subsection{Special Bôcher contractions}

Special Bôcher contractions are much easier to understand and manipulate than general Bôcher contractions: composition is merely matrix multiplication. The contractions that arise from the Bôcher recipe are not "special". However, we shall show that we can associate a special Bôcher contraction with each contraction obtained from Bôcher's recipe, such that the special contraction contains the same basic geometrical information. The (projective) tetraspherical coordinates are associated with points $(x, y)$ in $2 \mathrm{D}$ flat space via the relation

$$
(x, y) \equiv\left(x_{1}, x_{2}, x_{3}, x_{4}\right)=\left[x_{3}+i x_{4}\right]\left(-x,-y, \frac{1}{2}\left(1-x^{2}-y^{2}\right),-\frac{i}{2}\left(1+x^{2}+y^{2}\right)\right) .
$$

In particular,

$$
x=-\frac{x_{1}}{x_{3}+i x_{4}}, \quad y=-\frac{x_{2}}{x_{3}+i x_{4}}, \quad \frac{x_{3}+i x_{4}}{x_{3}-i x_{4}}=\frac{-1}{x^{2}+y^{2}} .
$$

For coordinates on the 2-sphere we have

$$
\left(s_{1}, s_{2}, s_{3}\right) \equiv\left(x_{1}, x_{2}, x_{3}, x_{4}\right)=x_{4}\left(-i s_{1},-i s_{2}, i s_{3}, 1\right) .
$$

The action of Bôcher contractions on the flat space coordinates $(x, y)$ is an affine mapping and this affine action carries all of the geometrical information about the contraction. For example, the $[1,1,1,1] \downarrow[2,1,1]$ contraction

$$
x_{3}=-\frac{i}{\sqrt{2} \epsilon} x_{3}^{\prime}-\frac{i}{\sqrt{2} \epsilon} x_{4}^{\prime}, \quad x_{4}=\frac{i}{\sqrt{2}}\left(\frac{1}{\epsilon}-\epsilon\right) x_{3}^{\prime}-\frac{1}{\sqrt{2}}\left(\frac{1}{\epsilon}+\epsilon\right) x_{4}^{\prime},
$$

and $x_{1}=x_{1}^{\prime}, x_{2}=x_{2}^{\prime}$, gives

$$
x=-\frac{x_{1}}{x_{3}+i x_{4}}=\frac{\epsilon x_{1}^{\prime}}{\sqrt{2}\left(x_{3}^{\prime}+i x_{4}^{\prime}\right)}+O\left(\epsilon^{2}\right)=\epsilon^{\prime} x^{\prime}+O\left(\epsilon^{\prime 2}\right), \quad y=\epsilon^{\prime} y^{\prime}+O\left(\epsilon^{\prime 2}\right),
$$

for $\epsilon^{\prime}=\epsilon / \sqrt{(2)}$. Thus the geometric content of the action of this contraction in flat space is $x=\epsilon^{\prime} x^{\prime}, y=\epsilon^{\prime} y^{\prime}$. The terms of order $\epsilon^{\prime 2}$ disappear in the limit. On the complex sphere we have

$$
\begin{aligned}
& s_{1}=\frac{i x_{1}}{x_{4}}=-\frac{\sqrt{2} i \epsilon x_{1}^{\prime}}{x_{3}^{\prime}+i x_{4}^{\prime}}+O\left(\epsilon^{2}\right)=\epsilon^{\prime} x^{\prime}+O\left(\epsilon^{\prime 2}\right), \quad s_{2}=\epsilon^{\prime} y^{\prime}+O\left(\epsilon^{2}\right), \\
& s_{3}=-\frac{i x_{3}}{x_{4}}=1+O\left(\epsilon^{2}\right)
\end{aligned}
$$

where $\epsilon^{\prime}=\sqrt{2} i \epsilon$ and $x^{\prime}, y^{\prime}$ are flat space coordinates. Thus the geometric content of the action of this contraction on the 2-sphere is $s_{1}=\epsilon^{\prime} x^{\prime}, s_{2}=\epsilon^{\prime} y^{\prime}$. Note that distinct contractions on flat space and the sphere are induced by the same Bôcher contraction.

Using the fact that the contraction limits are completely determined by the geometric limits, we can derive special Bôcher contractions that produce the same geometric limits. We again consider the example discussed above. We will design a special Bôcher contraction with the property $x=\epsilon x^{\prime}, y=\epsilon y^{\prime}$ such that equations (4.6), (4.7) hold. In this case we require $x=$ $x_{1} /\left(x_{3}+i x_{4}\right)=\epsilon x^{\prime}=\epsilon x_{1}^{\prime} /\left(x_{3}^{\prime}+i x_{4}^{\prime}\right), y=x_{2} /\left(x_{3}+i x_{4}\right)=x_{2}^{\prime} /\left(x_{3}^{\prime}+i x_{4}^{\prime}\right)$. The solution is, essentially unique up to conformal transformation:

$$
\begin{array}{ll}
x_{1}=x_{1}^{\prime}, & x_{3}=x_{3}^{\prime}(\epsilon+1 / \epsilon) / 2+i x_{4}^{\prime}(-\epsilon+1 / \epsilon) / 2, \\
x_{2}=x_{2}^{\prime}, & x_{4}=i x_{3}^{\prime}(\epsilon-1 / \epsilon) / 2+x_{4}^{\prime}(\epsilon+1 / \epsilon) / 2 .
\end{array}
$$


This contraction satisfies $x_{1}^{2}+x_{2}^{2}+x_{3}^{2}+x_{4}^{2}=x_{1}^{\prime 2}+x_{2}^{\prime 2}+x_{3}^{\prime 2}+x_{4}^{\prime 2}$ and agrees with $[1,1,1,1] \downarrow$ $[2,1,1]$ on Laplace equations.

Similarly we can use each of the geometric contractions of flat space and the 2-sphere as classified in [23], to construct special Bôcher contractions that take $V_{[1,1,1,1]}$ to each of $V_{[2,1,1]}$, $V_{[2,2]}, V_{[3,1]}, V_{[4]}$. For example

$$
\begin{aligned}
V_{[1,1,1,1]} \rightarrow V_{[3,1]}: \quad x_{1} & =x_{1}^{\prime}+\frac{x_{3}^{\prime}}{\epsilon}+\frac{i x_{4}^{\prime}}{\epsilon}, \quad x_{3}=-\frac{x_{1}^{\prime}}{\epsilon}+x_{3}^{\prime}\left(1-\frac{1}{2 \epsilon^{2}}\right)-\frac{i x_{4}^{\prime}}{2 \epsilon^{2}}, \\
x_{2} & =x_{2}^{\prime}, \quad x_{4}=-\frac{i x_{1}^{\prime}}{\epsilon}-\frac{i x_{3}^{\prime}}{2 \epsilon^{2}}+x_{4}^{\prime}\left(1+\frac{1}{2 \epsilon^{2}}\right) .
\end{aligned}
$$

A more general way to construct special Bôcher contractions is to make use of the normal forms for conjugacy classes of $\mathfrak{s o}(4, \mathbb{C})$ under the adjoint action of $\mathrm{SO}(4, \mathbb{C})$. They are derived in [9]:

$$
\begin{aligned}
C_{1} & =\left(\begin{array}{cccc}
0 & \lambda & 0 & 0 \\
-\lambda & 0 & 0 & 0 \\
0 & 0 & 0 & 0 \\
0 & 0 & 0 & 0
\end{array}\right), \quad C_{2}=\left(\begin{array}{cccc}
0 & \lambda & 0 & 0 \\
-\lambda & 0 & 0 & 0 \\
0 & 0 & 0 & \mu \\
0 & 0 & -\mu & 0
\end{array}\right), \\
C_{3} & =\left(\begin{array}{cccc}
0 & 1+i & 0 & 0 \\
-1-i & 0 & -1+i & 0 \\
0 & 1-i & 0 & 0 \\
0 & 0 & 0 & 0
\end{array}\right), \quad C_{4}=\frac{1}{2}\left(\begin{array}{cccc}
0 & 1 & i & 2 \lambda \\
-1 & 0 & 2 \lambda & i \\
-i & -2 \lambda & 0 & -1 \\
-2 \lambda & -i & 1 & 0
\end{array}\right) .
\end{aligned}
$$

Every 1-parameter subgroup $\mathbf{A}(t)$ of $\mathrm{SO}(4, \mathbb{C})$ (i.e., $\mathbf{A}\left(t_{1}+t_{2}\right)=\mathbf{A}\left(t_{1}\right) \mathbf{A}\left(t_{2}\right)$ ), is conjugate to one of the forms $\mathbf{A}_{j}(t)=\exp \left(t C_{j}\right), j=1,2,3,4$. By making an appropriate change of complex coordinate $t=t(\epsilon)$ we can obtain a special Bôcher contraction matrix

$$
\begin{aligned}
& \mathbf{A}_{1}(t)=\frac{1}{2}\left(\begin{array}{cccc}
\frac{\epsilon^{2}+1}{\epsilon} & -\frac{i\left(\epsilon^{2}-1\right)}{\epsilon} & 0 & 0 \\
\frac{i\left(\epsilon^{2}-1\right)}{\epsilon} & \frac{\epsilon^{2}+1}{\epsilon} & 0 & 0 \\
0 & 0 & 0 & 0 \\
0 & 0 & 0 & 0
\end{array}\right), \quad \epsilon=e^{i \lambda t}, \\
& \mathbf{A}_{2}(t)=\frac{1}{2}\left(\begin{array}{cccc}
\frac{\epsilon_{1}^{2}+1}{\epsilon_{1}} & -\frac{i\left(\epsilon_{1}^{2}-1\right)}{\epsilon_{1}} & 0 & 0 \\
\frac{i\left(\epsilon_{1}^{2}-1\right)}{\epsilon_{1}} & \frac{\epsilon_{1}^{2}+1}{\epsilon_{1}} & 0 & 0 \\
0 & 0 & \frac{\epsilon_{2}^{2}+1}{\epsilon_{2}} & -\frac{i\left(\epsilon_{2}^{2}-1\right)}{\epsilon_{2}} \\
0 & 0 & \frac{i\left(\epsilon_{2}^{2}-1\right)}{\epsilon_{2}} & \frac{\epsilon_{2}^{2}+1}{\epsilon_{2}}
\end{array}\right), \quad \epsilon_{1}=e^{i \lambda t}, \quad \epsilon_{2}=e^{i \mu t}, \\
& \mathbf{A}_{3}(t)=\left(\begin{array}{cccc}
1-\frac{1}{2 \epsilon^{2}} & \frac{1}{\epsilon} & \frac{i}{2 \epsilon^{2}} & 0 \\
-\frac{1}{\epsilon} & 1 & \frac{i}{\epsilon} & 0 \\
\frac{i}{2 \epsilon^{2}} & -\frac{i}{\epsilon} & 1+\frac{1}{2 \epsilon^{2}} & 0 \\
0 & 0 & 0 & 1
\end{array}\right), \quad \epsilon=\frac{2}{t(1+i)}, \\
& \mathbf{A}_{4}(t)=\frac{1}{2}\left(\begin{array}{cccc}
\frac{\epsilon_{1}^{2}+1}{\epsilon_{1}} & \frac{1}{\epsilon_{1} \epsilon_{2}} & \frac{i}{\epsilon_{1} \epsilon_{2}} & \frac{i\left(\epsilon_{1}^{2}-1\right)}{\epsilon_{1}} \\
-\frac{\epsilon_{1}}{\epsilon_{2}} & \frac{\epsilon_{1}^{2}+1}{\epsilon_{1}} & \frac{i\left(\epsilon_{1}^{2}-1\right)}{\epsilon_{1}} & \frac{i \epsilon_{1}}{\epsilon_{2}} \\
-\frac{i \epsilon_{1}}{\epsilon_{2}} & \frac{i\left(\epsilon_{1}^{2}-1\right)}{\epsilon_{1}} & \frac{\left.\epsilon_{1}^{2}+1\right)}{\epsilon_{1}} & -\frac{\epsilon_{1}}{\epsilon_{2}} \\
\frac{i\left(\epsilon_{1}^{2}-1\right)}{\epsilon_{1}} & \frac{i}{\epsilon_{1} \epsilon_{2}} & \frac{1}{\epsilon_{1} \epsilon_{2}} & \frac{\left.\epsilon_{1}^{2}+1\right)}{\epsilon_{1}}
\end{array}\right), \quad \epsilon_{1}=e^{i \lambda t}, \quad \epsilon_{2}=\frac{1}{t} .
\end{aligned}
$$

The contraction (4.8) takes $V_{[1,1,1,1]}$ to $V_{[2,11]},(4.9)$ takes it to $V_{[2,2]}$, and (4.10) takes it to $V_{[3,1]}$. The contractions (4.11), on the other hand, takes $V_{[1,1,1,1]}$ to $V_{[2,2]}$ again. Consider though the special case $\mathbf{H}(\epsilon)$, of (4.11) where $\epsilon_{1}=1, \epsilon_{2}=\epsilon$. It, too, maps $V_{[1,1,1,1]}$ to $V_{[2,2]}$, but the 
composition $\mathbf{H}(\epsilon) \mathbf{H}\left(\epsilon^{2}\right)$ takes $V_{[1,1,1,1]}$ to $V_{[4]}$. (We note that the composition $\mathbf{H}(\epsilon) \mathbf{H}\left(\epsilon^{3}\right)$ takes $V_{[1,1,1,1]}$ to $V_{[3,1]}$, showing that, in general, the result of a composition $\mathbf{A}\left(\epsilon_{1}\right) \mathbf{B}\left(\epsilon_{2}\right)$ depends on the relationship between $\epsilon_{1}$ and $\epsilon_{2}$.)

If the matrix $\mathbf{A}(\epsilon)$ defines a general Bôcher contraction, by transposing two rows if necessary, we can assume $\operatorname{det} \mathbf{A}(\epsilon)=1$ for all $\epsilon \neq 0$. Thus, $\mathbf{A}(\epsilon)$ is a curve on $\operatorname{SL}(4, \mathbb{C})$. We could use the results of [9] to list all the conjugacy classes of $\mathfrak{s l}(4, \mathbb{C})$ to attempt a classification. However, it would be necessary to check condition (4.3) in every case, whereas for special Bôcher contractions this condition is satisfied automatically.

Both Bôcher's original recipes and the normal forms given above provide a generating basis for all Bôcher contractions in two dimensions; the general contractions are obtained by composing these generators.

\section{Classification of free abstract nondegenerate quadratic algebras. Identification of those from free nondegenerate 2nd order superintegrable systems}

\subsection{Free nondegenerate classical quadratic algebras}

Recall from Definition 1.1 that the symmetry algebra of a free 2D superintegrable system on a constant curvature space, $\mathcal{A}$, is a quadratic algebra which is completely determined by the function $\mathcal{F}$. More specifically, it is a Poisson algebra generated by three linearly independent elements $\left\{\mathcal{L}_{1}, \mathcal{L}_{2}, \mathcal{H}\right\}$ where $\mathcal{H}$ generates the center of $\mathcal{A}$ and the structure equations of the algebra are given by (1.2) with

$$
\mathcal{R}^{2}=\mathcal{F}\left(\mathcal{H}, \mathcal{L}_{1}, \mathcal{L}_{2}\right)
$$

for some third order homogeneous polynomial $\mathcal{F}$. We call $R^{2}$, which is the same as $\mathcal{F}\left(\mathcal{H}, \mathcal{L}_{1}, \mathcal{L}_{2}\right)$, the Casimir of $\mathcal{A}$ in terms of $\left\{\mathcal{L}_{1}, \mathcal{L}_{2}, \mathcal{H}\right\}$. Motivated by the superintegrable case we define an abstract free nondegenerate $2 D$ classical quadratic algebra as follows.

Definition 5.1. A free nondegenerate $2 \mathrm{D}$ classical quadratic algebra is a Poisson algebra $\mathcal{A}$ over $\mathbb{C}$ that is generated by $\left\{\mathcal{L}_{1}, \mathcal{L}_{2}, \mathcal{H}\right\}$ where $\mathcal{H}$ generates the center of $\mathcal{A}$,

$$
\left\{\mathcal{R}, \mathcal{L}_{1}\right\}=-\frac{1}{2} \frac{\partial \mathcal{R}^{2}}{\partial \mathcal{L}_{2}}, \quad\left\{\mathcal{R}, \mathcal{L}_{2}\right\}=\frac{1}{2} \frac{\partial \mathcal{R}^{2}}{\partial \mathcal{L}_{1}}
$$

$\mathcal{R}=\left\{\mathcal{L}_{1}, \mathcal{L}_{2}\right\}$, and $\mathcal{R}^{2}=\mathcal{F}\left(\mathcal{H}, \mathcal{L}_{1}, \mathcal{L}_{2}\right)$ for some third order homogeneous polynomial $\mathcal{F}$.

Below we shall refer to free nondegenerate 2D classical quadratic algebras simply as (abstract) quadratic algebras.

Remark 5.2. As an associative algebra $\mathcal{A}$ is the quotient of the free $\mathbb{C}$-algebra generated by $\left\{\mathcal{L}_{1}, \mathcal{L}_{2}, \mathcal{H}, \mathcal{R}\right\}$ and its two sided ideal generated by $\mathcal{R}^{2}-\mathcal{F}$. For any choice of a polynomial of degree three for $\mathcal{F}$, the above equations define Lie brackets on $\mathcal{A}$ that make it a Poisson algebra, but higher order polynomials will not define Lie brackets on $\mathcal{A}$.

For any other generating set $\widetilde{\mathcal{L}}_{1}, \widetilde{\mathcal{L}}_{2}, \widetilde{\mathcal{H}}, \widetilde{\mathcal{R}}$ of the same Poisson algebra that satisfies:

(i) The linear span over $\mathbb{C}$ of $\widetilde{\mathcal{L}}_{1}, \widetilde{\mathcal{L}}_{2}, \widetilde{\mathcal{H}}$ coincides with the linear span of $\mathcal{L}_{1}, \mathcal{L}_{2}, \mathcal{H}$.

(ii) $\widetilde{\mathcal{H}}$ is in the center of the Poisson algebra, i.e., Poisson commutes with everything.

(iii) $\widetilde{\mathcal{R}}=\left\{\widetilde{\mathcal{L}}_{1}, \widetilde{\mathcal{L}}_{2}\right\}$. 
(iv) The generators $\widetilde{\mathcal{L}}_{1}, \widetilde{\mathcal{L}}_{2}, \widetilde{\mathcal{H}}, \widetilde{\mathcal{R}}$ satisfy the structure equations, i.e.,

$$
\left\{\widetilde{\mathcal{R}}, \widetilde{\mathcal{L}}_{1}\right\}=-\frac{1}{2} \frac{\partial \widetilde{\mathcal{R}}^{2}}{\partial \widetilde{\mathcal{L}}_{2}}, \quad\left\{\widetilde{\mathcal{R}}, \widetilde{\mathcal{L}}_{2}\right\}=\frac{1}{2} \frac{\partial \widetilde{\mathcal{R}}^{2}}{\partial \widetilde{\mathcal{L}}_{1}} .
$$

It easy to see that

$$
\left(\begin{array}{c}
\widetilde{\mathcal{L}}_{1} \\
\widetilde{\mathcal{L}}_{2} \\
\widetilde{\mathcal{H}}
\end{array}\right)=\left(\begin{array}{ccc}
A_{1,1} & A_{1,2} & A_{1,3} \\
A_{2,1} & A_{2,2} & A_{2,3} \\
0 & 0 & A_{3,3}
\end{array}\right)\left(\begin{array}{c}
\mathcal{L}_{1} \\
\mathcal{L}_{2} \\
\mathcal{H}
\end{array}\right)
$$

for some

$$
A=\left(\begin{array}{ccc}
A_{1,1} & A_{1,2} & A_{1,3} \\
A_{2,1} & A_{2,2} & A_{2,3} \\
0 & 0 & A_{3,3}
\end{array}\right) \in \operatorname{GL}(3, \mathbb{C}) .
$$

For a matrix as above we define $A_{2}=\left(\begin{array}{ll}A_{1,1} & A_{1,2} \\ A_{2,1} & A_{2,2}\end{array}\right) \in \mathrm{GL}(2, \mathbb{C})$. We denote the group of matrices of the form (5.2) by $G$, it is a complex algebraic group. Moreover, if $\mathcal{R}^{2}=\mathcal{F}$ and $\tilde{\mathcal{R}}^{2}=\tilde{\mathcal{F}}$ then there is $A \in G$, such that

$$
\widetilde{\mathcal{F}}\left(\widetilde{\mathcal{L}}_{1}, \widetilde{\mathcal{L}}_{2}, \widetilde{\mathcal{H}}\right)=\operatorname{det}\left(A_{2}\right)^{2} \mathcal{F}\left(A^{-1}\left(\widetilde{\mathcal{L}}_{1}, \widetilde{\mathcal{L}}_{2}, \widetilde{\mathcal{H}}\right)\right) .
$$

Obviously, two quadratic algebras are isomorphic if and only if their Casimirs are related by $A \in G$ via equation (5.3). This fact is fundamental for the classification of quadratic algebras.

Let $\mathbb{C}^{[3]}\left[x_{1}, x_{2}, x_{3}\right]$ be the complex algebraic variety of homogeneous polynomials of degree three in the variables $x_{1}, x_{2}, x_{3}$. The group $G$ acts on $\mathbb{C}^{[3]}\left[x_{1}, x_{2}, x_{3}\right]$ via equation (5.3). Obviously there is a bijection between isomorphism classes of quadratic algebras and orbits of $G$ in $\mathbb{C}^{[3]}\left[x_{1}, x_{2}, x_{3}\right]$. We will determine all isomorphism classes of quadratic algebras by classifying all orbits of $G$ in $\mathbb{C}^{[3]}\left[x_{1}, x_{2}, x_{3}\right]$. We shall distinguish an element in each orbit that defines the Canonical form for the Casimir of a given quadratic algebra. Moreover we present an algorithm for finding the canonical form of the Casimir for a given quadratic algebra which gives a practical way to determine if two given quadratic algebras are isomorphic.

\subsection{The algorithm for casting the Casimir to its the canonical form}

In this section we introduce the notation $X_{1}=\mathcal{L}_{1}, X_{2}=\mathcal{L}_{2}, X_{3}=\mathcal{H}$ and similarly, $\widetilde{X}_{1}=$ $\widetilde{\mathcal{L}}_{1}, \widetilde{X}_{2}=\widetilde{\mathcal{L}}_{2}, \widetilde{X}_{3}=\widetilde{\mathcal{H}}$. For any realization of the Casimir, $R^{2}=\mathcal{F}\left(X_{1}, X_{2}, X_{3}\right)$, there are homogeneous polynomials in $X_{1}, X_{2}$ of order $j, \mathcal{F}^{(j)}$, such that

$$
\mathcal{F}\left(X_{1}, X_{2}, X_{3}\right)=\mathcal{F}^{(3)}\left(X_{1}, X_{2}\right)+X_{3} \mathcal{F}^{(2)}\left(X_{1}, X_{2}\right)+X_{3}{ }^{2} \mathcal{F}^{(1)}\left(X_{1}, X_{2}\right)+X_{3}{ }^{3} \mathcal{F}^{(0)} .
$$

For any $f \in \mathbb{C}^{[3]}\left[X_{1}, X_{2}, X_{3}\right]$ we shall denote the stabilizer of $f$ in $G$ by $\operatorname{Stab}_{G}\{f\}$. We shall use the notation $\operatorname{Stab}_{G}\{f+O(\mathcal{H})\}$ for the subgroup of $G$ consisting of all elements that do not change the part in $f$ that is $\mathcal{H}$ independent. That is $g \in \operatorname{Stab}_{G}\{f+O(\mathcal{H})\}$ preserves the lowest order term in $f$ as a polynomial of $\mathcal{H}=X_{3}$. Similarly $\operatorname{Stab}_{G}\left\{f+O\left(\mathcal{H}^{2}\right)\right\}$ stands for the subgroup of $G$ consisting of all elements that preserves the part in $f$ that is a polynomial of degree 1 in $\mathcal{H}$. Similarly we define $\operatorname{Stab}_{G}\left\{f+O\left(\mathcal{H}^{3}\right)\right\}$. For a given $f \in \mathbb{C}^{[3]}\left[X_{1}, X_{2}, X_{3}\right]$ we shall denote by $f^{(i)}\left(X_{1}, X_{2}\right)$ it homogeneous component that are uniquely defined by

$$
f\left(X_{1}, X_{2}, X_{3}\right)=f^{(3)}\left(X_{1}, X_{2}\right)+X_{3} f^{(2)}\left(X_{1}, X_{2}\right)+X_{3}^{2} f^{(1)}\left(X_{1}, X_{2}\right)+X_{3}^{3} f^{(0)} .
$$


Note that

$$
\begin{aligned}
\operatorname{Stab}_{G} & \left\{f^{(3)}+O(\mathcal{H})\right\} \supseteq \operatorname{Stab}_{G}\left\{f^{(3)}+\mathcal{H} f^{(2)}+O\left(\mathcal{H}^{2}\right)\right\} \\
\supseteq & \operatorname{Stab}_{G}\left\{f^{(3)}+\mathcal{H} f^{(2)}+\mathcal{H}^{2} f^{(1)}+O\left(\mathcal{H}^{3}\right)\right\} \supseteq \operatorname{Stab}_{G}\{f\} .
\end{aligned}
$$

The algorithm for casting $R^{2}=\mathcal{F}\left(X_{1}, X_{2}, X_{3}\right)$ into its canonical form is as follows:

Step1 Using a certain $g_{1} \in G$ we transform $\mathcal{F}\left(X_{1}, X_{2}, X_{3}\right)$ to a form in which $\mathcal{F}^{(3)}$ is in a canonical form, $\mathcal{F}_{c}^{(3)}$.

Step2 Using a certain $g_{2} \in \operatorname{Stab}_{G}\left\{\mathcal{F}_{c}^{(3)}+O(\mathcal{H})\right\}$ we transform $\mathcal{F}\left(X_{1}, X_{2}, X_{3}\right)$ (that we got in step 1) to a form in which $\mathcal{F}^{(3)}+\mathcal{H F}^{(2)}$ is in a canonical form $\mathcal{F}_{c}^{(3)}+\mathcal{H F}_{c}^{(2)}$.

Step3 Using a certain $g_{3} \in \operatorname{Stab}_{G}\left\{\mathcal{F}_{c}^{(3)}+\mathcal{H}_{c}^{(2)}+O\left(\mathcal{H}^{2}\right)\right\}$ we transform $\mathcal{F}\left(X_{1}, X_{2}, X_{3}\right)$ (that we got in step 2) to a form in which $\mathcal{F}^{(3)}+\mathcal{H F}^{(2)}+\mathcal{H}^{2} \mathcal{F}^{(1)}$ is in a canonical form $\mathcal{F}_{c}^{(3)}+$ $\mathcal{H} \mathcal{F}_{c}^{(2)}+\mathcal{H}^{2} \mathcal{F}_{c}^{(1)}$.

Step4 Using a certain $g_{4} \in \operatorname{Stab}_{G}\left\{\mathcal{F}_{c}^{(3)}+\mathcal{H} \mathcal{F}_{c}^{(2)}+\mathcal{H}^{2} \mathcal{F}_{c}^{(1)}+O\left(\mathcal{H}^{3}\right)\right\}$ we transform $\mathcal{F}\left(X_{1}, X_{2}, X_{3}\right)$ (that we got in step 3) to a form in which $\mathcal{F}^{(3)}+\mathcal{H} \mathcal{F}^{(2)}+\mathcal{H}^{2} \mathcal{F}^{(1)}+\mathcal{H}^{3} \mathcal{F}^{(0)}$ is in a canonical form $\mathcal{F}_{c}^{(3)}+\mathcal{H} \mathcal{F}_{c}^{(2)}+\mathcal{H}^{2} \mathcal{F}_{c}^{(1)}+\mathcal{H}^{3} \mathcal{F}_{c}^{(0)}$. This is the canonical form of $\mathcal{F}$.

At the end of the section we list all possible canonical form of quadratic algebras in a table.

\subsubsection{The four cases for $\mathcal{F}^{(3)}$}

Note that for two presentations of the Casimir of a given quadratic algebra: $R^{2}=\mathcal{F}\left(X_{1}, X_{2}, X_{3}\right)$ and $\widetilde{\mathcal{R}}^{2}=\widetilde{\mathcal{F}}\left(\widetilde{X}_{1}, \widetilde{X}_{2}, \widetilde{X}\right)$ that are related by equation (5.1) with $A=\left(\begin{array}{ccc}A_{1,1} & A_{1,2} & 0 \\ A_{2,1} & A_{2,2} & 0 \\ 0 & 0 & 1\end{array}\right) \in$ $\mathrm{GL}(3, \mathbb{C})$ and

$$
\widetilde{\mathcal{R}}^{2}=\widetilde{\mathcal{F}}^{(3)}\left(\widetilde{X}_{1}, \widetilde{X}_{2}\right)+\widetilde{X}_{3} \widetilde{\mathcal{F}}^{(2)}\left(\widetilde{X}_{1}, \widetilde{X}_{2}\right)+\widetilde{X}_{3}^{2} \widetilde{\mathcal{F}}^{(1)}\left(\widetilde{X}_{1}, \widetilde{X}_{2}\right)+\widetilde{X}_{3}^{3} \widetilde{\mathcal{F}}^{(0)}
$$

we have

$$
\widetilde{\mathcal{F}}^{(i)}\left(\widetilde{X}_{1}, \widetilde{X}_{2}\right)=\operatorname{det}\left(A_{2}\right)^{2} \mathcal{F}^{(i)}\left(A_{2}^{-1}\left(\widetilde{X}_{1}, \widetilde{X}_{2}\right)\right) .
$$

From this we can deduce the following lemma.

Lemma 5.3. Given $\mathcal{F} \in \mathbb{C}^{[3]}\left[x_{1}, x_{2}, x_{3}\right]$ we can find an explicit matrix $A \in G$ such that for

$$
\widetilde{\mathcal{F}}\left(\widetilde{\mathcal{L}}_{1}, \widetilde{\mathcal{L}}_{2}, \widetilde{\mathcal{H}}\right)=\operatorname{det}\left(A_{2}\right)^{2} \mathcal{F}\left(A^{-1}\left(\widetilde{\mathcal{L}}_{1}, \widetilde{\mathcal{L}}_{2}, \widetilde{\mathcal{H}}\right)\right)
$$

we have $\widetilde{\mathcal{F}}^{(3)}\left(\widetilde{X}_{1}, \widetilde{X}_{2}\right)=C_{I}\left(X_{1}, X_{2}\right)$, where $C_{I}$ equal to exactly one of the following

$$
0, \quad C_{1}\left(X_{1}, X_{2}\right)=X_{1} X_{2}\left(X_{1}+X_{2}\right), \quad C_{2}\left(X_{1}, X_{2}\right)=X_{1}^{2} X_{2}, \quad C_{3}\left(X_{1}, X_{2}\right)=X_{1}^{3} \text {. }
$$

\section{Proposition 5.4.}

$$
\operatorname{Stab}_{G}\left(C_{1}+O(\mathcal{H})\right)=\left\{\left(\begin{array}{cc}
A & v \\
0 & c
\end{array}\right) \mid A \in \Omega\left(C_{1}\right), v \in \mathbb{C}^{2}, c \in \mathbb{C}^{*}\right\}
$$


where

$$
\begin{gathered}
\Omega\left(C_{1}\right)=\left\{\left(\begin{array}{ll}
0 & 1 \\
1 & 0
\end{array}\right),\left(\begin{array}{cc}
0 & 1 \\
-1 & -1
\end{array}\right),\left(\begin{array}{ll}
1 & 0 \\
0 & 1
\end{array}\right)\right\} \\
\coprod\left\{\left(\begin{array}{cc}
-1 & -1 \\
0 & -1
\end{array}\right),\left(\begin{array}{cc}
1 & 0 \\
-1 & -1
\end{array}\right),\left(\begin{array}{cc}
-1 & -1 \\
1 & 0
\end{array}\right)\right\}, \\
\operatorname{Stab}_{G}\left(C_{2}+O(\mathcal{H})\right)=\left\{\left(\begin{array}{ccc}
a & 0 & v_{1} \\
0 & 1 & v_{2} \\
0 & 0 & c
\end{array}\right) \mid v_{1}, v_{2} \in \mathbb{C}, a, c \in \mathbb{C}^{*}\right\}, \\
\operatorname{Stab}_{G}\left(C_{3}+O(\mathcal{H})\right)=\left\{\left(\begin{array}{ccc}
d^{2} & 0 & v_{1} \\
b & d & v_{2} \\
0 & 0 & c
\end{array}\right) \mid b, v_{1}, v_{2} \in \mathbb{C}, c, d \in \mathbb{C}^{*}\right\} .
\end{gathered}
$$

\subsection{First case: three distinct roots}

Suppose that

$$
\mathcal{F}^{(3)}\left(X_{1}, X_{2}\right)+\mathcal{H} \mathcal{F}^{(2)}\left(X_{1}, X_{2}\right)=C_{1}\left(X_{1}, X_{2}\right)+\mathcal{H}\left(c_{5} X_{1}^{2}+c_{6} X_{2}^{2}+c_{7} X_{1} X_{2}\right) .
$$

Acting with

$$
A=\left(\begin{array}{ccc}
1 & 0 & -c_{6} \\
0 & 1 & -c_{5} \\
0 & 0 & 1
\end{array}\right)^{-1} \in \operatorname{Stab}_{G}\left(C_{1}\left(X_{1}, X_{2}\right)\right)
$$

we get

$$
\begin{aligned}
C_{1}\left(X_{1}, X_{2}\right)+\mathcal{H}\left(c_{5} X_{1}^{2}+c_{6} X_{2}^{2}+c_{7} X_{1} X_{2}\right) \\
\quad \longmapsto C_{1}\left(X_{1}, X_{2}\right)+\mathcal{H}\left(c_{7}^{\prime} X_{1} X_{2}\right)+\mathcal{H}^{2}\left(c_{8}^{\prime} X_{1}+c_{9}^{\prime} X_{2}\right)+c_{10} \mathcal{H}^{3}
\end{aligned}
$$

for some $c_{7}^{\prime}, c_{8}^{\prime}, c_{9}^{\prime}, c_{10}^{\prime}$, hence we can assume that the

$$
\mathcal{F}^{(3)}\left(X_{1}, X_{2}\right)+\mathcal{H} \mathcal{F}^{(2)}\left(X_{1}, X_{2}\right)=C_{1}\left(X_{1}, X_{2}\right)+c_{7} \mathcal{H} X_{1} X_{2}+O\left(\mathcal{H}^{2}\right)
$$

using a matrix of the form

$$
A=\left(\begin{array}{lll}
1 & 0 & 0 \\
0 & 1 & 0 \\
0 & 0 & r
\end{array}\right)
$$

we can further assume that $c_{7} \in\{0,1\}$. For the case of $c_{7}=0$ we obtain the following proposition:

Proposition 5.5. The stabilizer of the form

$$
\mathcal{F}^{(3)}\left(X_{1}, X_{2}\right)+\mathcal{H} \mathcal{F}^{(2)}\left(X_{1}, X_{2}\right)+O\left(\mathcal{H}^{2}\right)=C_{1}\left(X_{1}, X_{2}\right)+O\left(\mathcal{H}^{2}\right)
$$

is given by

$$
\operatorname{Stab}_{G}\left(C_{1}\left(X_{1}, X_{2}\right)+O\left(\mathcal{H}^{2}\right)\right)=\left\{\left(\begin{array}{cc}
A & 0_{2} \\
0 & c
\end{array}\right) \mid A \in \Omega\left(C_{1}\right), 0_{2}=0 \in \mathbb{C}^{2}, c \in \mathbb{C}^{*}\right\} .
$$


Proof. It is easy to see that

$$
\operatorname{Stab}_{G}\left(C_{1}\left(X_{1}, X_{2}\right)+O\left(\mathcal{H}^{2}\right)\right) \supseteq\left\{\left(\begin{array}{cc}
A & 0_{2} \\
0 & c
\end{array}\right) \mid A \in \Omega\left(C_{1}\right), 0_{2}=0 \in \mathbb{C}^{2}, c \in \mathbb{C}^{*}\right\} .
$$

For inclusion in the other direction, let $M \in \operatorname{Stab}_{G}\left(C_{1}\left(X_{1}, X_{2}\right)+O\left(\mathcal{H}^{2}\right)\right)$ then obviously $M_{2}$ has to preserve $C_{1}\left(X_{1}, X_{2}\right)$, i.e., $M_{2} \in \Omega\left(C_{1}\right)$. Hence the matrix

$$
\left(\begin{array}{ccc}
\left(M^{-1}\right)_{1,1} & \left(M^{-1}\right)_{1,2} & 0 \\
\left(M^{-1}\right)_{2,1} & \left(M^{-1}\right)_{2,2} & 0 \\
0 & 0 & 1
\end{array}\right) M=\left(\begin{array}{ccc}
1 & 0 & M_{1,3} \\
0 & 1 & M_{2,3} \\
0 & 0 & M_{3,3}
\end{array}\right)
$$

as a product of two matrices in the stabilizer $\operatorname{Stab}_{G}\left(C_{1}\left(X_{1}, X_{2}\right)+O\left(\mathcal{H}^{2}\right)\right)$ is also in the stabilizer. The result of the action of this matrix on $C_{1}\left(X_{1}, X_{2}\right)+O\left(\mathcal{H}^{2}\right)$ forces $M_{1,3}=M_{2,3}=0$.

For the case of $c_{7}=1$ we obtain the following proposition:

Proposition 5.6. The stabilizer of the form

$$
\mathcal{F}^{(3)}\left(X_{1}, X_{2}\right)+\mathcal{H} \mathcal{F}^{(2)}\left(X_{1}, X_{2}\right)+O\left(\mathcal{H}^{2}\right)=C_{1}\left(X_{1}, X_{2}\right)+\mathcal{H} X_{1} X_{2}+O\left(\mathcal{H}^{2}\right)
$$

is given by

$$
\operatorname{Stab}_{G}\left(C_{1}\left(X_{1}, X_{2}\right)+\mathcal{H} X_{1} X_{2}+O\left(\mathcal{H}^{2}\right)\right)=\left\{\left(\begin{array}{lll}
1 & 0 & 0 \\
0 & 1 & 0 \\
0 & 0 & 1
\end{array}\right)\right\}
$$

Proof. Following the same reasoning as in the previous proof we easily see that for $M \in$ $\operatorname{Stab}_{G}\left(C_{1}\left(X_{1}, X_{2}\right)+\mathcal{H} X_{1} X_{2}+O\left(\mathcal{H}^{2}\right)\right)$ we must have $M_{2}=\left(\begin{array}{ll}1 & 0 \\ 0 & 1\end{array}\right)$ and then by direct calculation the rest of the proof follows.

\subsection{1 $\mathcal{F}^{(3)}\left(X_{1}, X_{2}\right)=C_{1}\left(X_{1}, X_{2}\right)$ and $c_{7}=0$}

Suppose that

$$
\begin{aligned}
& \mathcal{R}^{2}=\mathcal{F}^{(3)}\left(X_{1}, X_{2}\right)+\mathcal{H} \mathcal{F}^{(2)}\left(X_{1}, X_{2}\right)+\mathcal{H}^{2} \mathcal{F}^{(1)}\left(X_{1}, X_{2}\right)+c_{10} \mathcal{H}^{3} \\
& =C_{1}\left(X_{1}, X_{2}\right)+\mathcal{H}^{2}\left(c_{8} X_{1}+c_{9} X_{2}\right)+c_{10} \mathcal{H}^{3} \text {. } \\
& \text { Acting with } A=\left(\begin{array}{ccc}
\alpha & \beta & 0 \\
\gamma & \delta & 0 \\
0 & 0 & c
\end{array}\right)^{-1} \in \operatorname{Stab}_{G}\left(C_{1}\left(X_{1}, X_{2}\right)+O\left(\mathcal{H}^{2}\right)\right) \text { on } \mathcal{R}^{2} \text { we will have } \\
& \mathcal{R}^{2}=C_{1}\left(X_{1}, X_{2}\right)+\mathcal{H}^{2}\left(c_{8} X_{1}+c_{9} X_{2}\right)+c_{10} \mathcal{H}^{3} \\
& \longmapsto C_{1}\left(X_{1}, X_{2}\right)+\mathcal{H}^{2}\left(c_{8}^{\prime} X_{1}+c_{9}^{\prime} X_{2}\right)+c_{10}^{\prime} \mathcal{H}^{3},
\end{aligned}
$$

where $c_{8}^{\prime}=c^{2}\left(\alpha c_{8}+\gamma c_{9}\right), c_{9}^{\prime}=c^{2}\left(\beta c_{8}+\delta c_{9}\right), c_{10}^{\prime}=c^{3} c_{10}$, and $\left(\begin{array}{ll}\alpha & \beta \\ \gamma & \delta\end{array}\right) \in \Omega\left(C_{1}\right)$. Note that the size of the group $\Omega\left(C_{1}\right)$ is 6 .

We now describe an algorithm for choosing a canonical form in this case. If $c_{10} \neq 0$ then $\operatorname{acting}$ with $\left(\begin{array}{ccc}1 & 0 & 0 \\ 0 & 1 & 0 \\ 0 & 0 & \left(c_{10}\right)^{\frac{1}{3}}\end{array}\right)$ we obtain $c_{10}^{\prime}=1$. Writing $\left(\begin{array}{c}c_{8}^{\prime} \\ c_{9}^{\prime}\end{array}\right)=\left(\begin{array}{c}r e^{i \theta} \\ \rho e^{i \phi}\end{array}\right)$ with $r, \rho \geq 0$ and 
$\theta, \phi \in[0,2 \pi)$ we choose as our canonical form the expression for $c_{8}$ and $c_{9}$ according to the following rules (note that the order is important) first make $r$ is maximal, then $\theta$ minimal, then $\rho$ minimal, and finally $\phi$ minimal. If $c_{10}=0$ then again we act with $A=\left(\begin{array}{ccc}\alpha & \beta & 0 \\ \gamma & \delta & 0 \\ 0 & 0 & 1\end{array}\right)^{-1}$ with $\left(\begin{array}{ll}\alpha & \beta \\ \gamma & \delta\end{array}\right) \in \Omega\left(C_{1}\right)$ and choose $c_{8}$ and $c_{9}$ as above and then we can act with a matrix of the form $\left(\begin{array}{lll}1 & 0 & 0 \\ 0 & 1 & 0 \\ 0 & 0 & c\end{array}\right)$ to normalize $c_{8}$ to zero or one.

\subsection{2 $\mathcal{F}^{(3)}\left(X_{1}, X_{2}\right)=C_{1}\left(X_{1}, X_{2}\right)$ and $c_{7}=1$}

Suppose that

$$
\begin{aligned}
\mathcal{R}^{2} & =\mathcal{F}^{(3)}\left(X_{1}, X_{2}\right)+\mathcal{H} \mathcal{F}^{(2)}\left(X_{1}, X_{2}\right)+\mathcal{H}^{2} \mathcal{F}^{(1)}\left(X_{1}, X_{2}\right)+c_{10} \mathcal{H}^{3} \\
& =C_{1}\left(X_{1}, X_{2}\right)+\mathcal{H} X_{1} X_{2}+\mathcal{H}^{2}\left(c_{8} X_{1}+c_{9} X_{2}\right)+c_{10} \mathcal{H}^{3} .
\end{aligned}
$$

Since

$$
\operatorname{Stab}_{G}\left(C_{1}\left(X_{1}, X_{2}\right)+\mathcal{H} X_{1} X_{2}+O\left(\mathcal{H}^{2}\right)\right)=\left\{\left(\begin{array}{lll}
1 & 0 & 0 \\
0 & 1 & 0 \\
0 & 0 & 1
\end{array}\right)\right\}
$$

then for any $c_{8}, c_{9}, c_{10} \in \mathbb{C}$ equation (5.4) defines a canonical form.

\subsection{Second case: a double root}

Suppose that

$$
\mathcal{F}^{(3)}\left(X_{1}, X_{2}\right)+\mathcal{H} \mathcal{F}^{(2)}\left(X_{1}, X_{3}\right)=C_{2}\left(X_{1}, X_{2}\right)+\mathcal{H}\left(c_{5} X_{1}^{2}+c_{6} X_{2}^{2}+c_{7} X_{1} X_{2}\right) .
$$

Acting with

$$
A=\left(\begin{array}{ccc}
1 & 0 & -\frac{1}{2} c_{7} \\
0 & 1 & -c_{5} \\
0 & 0 & 1
\end{array}\right)^{-1} \in \operatorname{Stab}_{G}\left(C_{2}\left(X_{1}, X_{2}\right)\right)
$$

on $\mathcal{R}^{2}$ we have

$$
\begin{aligned}
& C_{2}\left(X_{1}, X_{2}\right)+\mathcal{H}\left(c_{5} X_{1}^{2}+c_{6} X_{2}^{2}+c_{7} X_{1} X_{2}\right) \\
& \quad \longmapsto C_{2}\left(X_{1}, X_{2}\right)+\mathcal{H}\left(c_{6}^{\prime} X_{2}^{2}\right)+\mathcal{H}^{2}\left(c_{8}^{\prime} X_{1}+c_{9}^{\prime} X_{2}\right)+c_{10}^{\prime} \mathcal{H}^{3}
\end{aligned}
$$

for some $c_{6}^{\prime}, c_{8}^{\prime}, c_{9}^{\prime}, c_{10}^{\prime}$. Hence we can assume that the

$$
\mathcal{F}^{(3)}\left(X_{1}, X_{2}\right)+\mathcal{H F}^{(2)}\left(X_{1}, X_{3}\right)=C_{2}\left(X_{1}, X_{2}\right)+c_{6} \mathcal{H} X_{2}^{2}+O\left(\mathcal{H}^{2}\right)
$$

using a matrix of the form $A=\left(\begin{array}{lll}1 & 0 & 0 \\ 0 & 1 & 0 \\ 0 & 0 & r\end{array}\right)$ we can further assume that $c_{6} \in\{0,1\}$. For the case of $c_{6}=0$ we obtain the following proposition: 
Proposition 5.7. The stabilizer of the form

$$
\mathcal{F}^{(3)}\left(X_{1}, X_{2}\right)+\mathcal{H} \mathcal{F}^{(2)}\left(X_{1}, X_{2}\right)+O\left(\mathcal{H}^{2}\right)=C_{2}\left(X_{1}, X_{2}\right)+O\left(\mathcal{H}^{2}\right)
$$

is given by

$$
\operatorname{Stab}_{G}\left(C_{2}\left(X_{1}, X_{2}\right)+O\left(\mathcal{H}^{2}\right)\right)=\left\{\left(\begin{array}{lll}
a & 0 & 0 \\
0 & 1 & 0 \\
0 & 0 & c
\end{array}\right) \mid a, c \in \mathbb{C}^{*}\right\}
$$

For the case of $c_{6}=1$ we obtain the following proposition:

Proposition 5.8. The stabilizer of the form

$$
\mathcal{F}^{(3)}\left(X_{1}, X_{2}\right)+\mathcal{H} \mathcal{F}^{(2)}\left(X_{1}, X_{2}\right)+O\left(\mathcal{H}^{2}\right)=C_{2}\left(X_{1}, X_{2}\right)+\mathcal{H} X_{2}^{2}+O\left(\mathcal{H}^{2}\right)
$$

is given by

$$
\operatorname{Stab}_{G}\left(C_{2}\left(X_{1}, X_{2}\right)+\mathcal{H} X_{2}^{2}+O\left(\mathcal{H}^{2}\right)\right)=\left\{\left(\begin{array}{ccc}
r & 0 & 0 \\
0 & 1 & 0 \\
0 & 0 & r^{2}
\end{array}\right) \mid r \in \mathbb{C}^{*}\right\} .
$$

\subsection{1 $\mathcal{F}^{(3)}\left(X_{1}, X_{2}\right)=C_{2}\left(X_{1}, X_{2}\right)$ and $c_{6}=0$}

Suppose that

$$
\begin{aligned}
& \qquad \begin{array}{l}
\mathcal{R}^{2}=\mathcal{F}^{(3)}\left(X_{1}, X_{2}\right)+\mathcal{H} \mathcal{F}^{(2)}\left(X_{1}, X_{2}\right)+\mathcal{H}^{2} \mathcal{F}^{(1)}\left(X_{1}, X_{2}\right)+c_{10} \mathcal{H}^{3} \\
=C_{2}\left(X_{1}, X_{2}\right)+\mathcal{H}^{2}\left(c_{8} X_{1}+c_{9} X_{2}\right)+c_{10} \mathcal{H}^{3} .
\end{array} \\
& \text { Acting with } A=\left(\begin{array}{lll}
a & 0 & 0 \\
0 & 1 & 0 \\
0 & 0 & c
\end{array}\right)^{-1} \in \operatorname{Stab}_{G}\left(C_{2}\left(X_{1}, X_{2}\right)+O\left(\mathcal{H}^{2}\right)\right) \text { on } \mathcal{R}^{2} \text { we have } \\
& \mathcal{R}^{2}=C_{2}\left(X_{1}, X_{2}\right)+\mathcal{H}^{2}\left(c_{8} X_{1}+c_{9} X_{2}\right)+c_{10} \mathcal{H}^{3} \\
& \quad \longmapsto C_{2}\left(X_{1}, X_{2}\right)+\mathcal{H}^{2}\left(c_{8}^{\prime} X_{1}+c_{9}^{\prime} X_{2}\right)+c_{10}^{\prime} \mathcal{H}^{3},
\end{aligned}
$$

where $c_{8}^{\prime}=c^{2} a^{-1} c_{8}, c_{9}^{\prime}=c^{2} a^{-2} c_{9}, c_{10}^{\prime}=c^{3} a^{-2} c_{10}$. For the canonical form, we normalize the first two non zero coefficients from $c_{8}, c_{9}, c_{10}$ to be equal to 1 .

\subsection{2 $\mathcal{F}^{(3)}\left(X_{1}, X_{2}\right)=C_{2}\left(X_{1}, X_{2}\right)$ and $c_{6}=1$}

Suppose that

$$
\mathcal{R}^{2}=C_{2}\left(X_{1}, X_{2}\right)+\mathcal{H} X_{2}^{2}+\mathcal{H}^{2}\left(c_{8} X_{1}+c_{9} X_{2}\right)+c_{10} \mathcal{H}^{3} .
$$

Acting with $A=\left(\begin{array}{ccc}r & 0 & 0 \\ 0 & 1 & 0 \\ 0 & 0 & r^{2}\end{array}\right)^{-1} \in \operatorname{Stab}_{G}\left(C_{2}\left(X_{1}, X_{2}\right)+\mathcal{H} X_{2}^{2}+O\left(\mathcal{H}^{2}\right)\right)$ on $\mathcal{R}^{2}$ we have

$$
\begin{aligned}
\mathcal{R}^{2} & =C_{2}\left(X_{1}, X_{2}\right)+\mathcal{H} X_{2}^{2}++\mathcal{H}^{2}\left(c_{8} X_{1}+c_{9} X_{2}\right)+c_{10} \mathcal{H}^{3} \\
& \longmapsto C_{2}\left(X_{1}, X_{2}\right)+\mathcal{H} X_{2}^{2}+\mathcal{H}^{2}\left(c_{8}^{\prime} X_{1}+c_{9}^{\prime} X_{2}\right)+c_{10}^{\prime} \mathcal{H}^{3},
\end{aligned}
$$

where $c_{8}^{\prime}=r^{3} c_{8}, c_{9}^{\prime}=r^{2} c_{9}, c_{10}^{\prime}=r^{4} c_{10}$. We define the canonical form to be with $c_{k}=1$, where $k$ is the smallest integer among $\{8,9,10\}$ such that $c_{k} \neq 0$. 


\subsection{Third case: a triple root}

Suppose that

$$
\mathcal{F}^{(3)}\left(X_{1}, X_{2}\right)+\mathcal{H} \mathcal{F}^{(2)}\left(X_{1}, X_{2}\right)=C_{3}\left(X_{1}, X_{2}\right)+\mathcal{H}\left(c_{5} X_{1}^{2}+c_{6} X_{2}^{2}+c_{7} X_{1} X_{2}\right) .
$$

Acting with

$$
A=\left(\begin{array}{ccc}
1 & 0 & -\frac{1}{3} c_{5} \\
0 & 1 & 0 \\
0 & 0 & 1
\end{array}\right)^{-1} \in \operatorname{Stab}_{G}\left(C_{3}\left(X_{1}, X_{2}\right)\right)
$$

on $\mathcal{R}^{2}$ we have

$$
\begin{aligned}
C_{3}\left(X_{1}, X_{2}\right)+\mathcal{H}\left(c_{5} X_{1}^{2}+c_{6} X_{2}^{2}+c_{7} X_{1} X_{2}\right) \\
\quad \longmapsto C_{3}\left(X_{1}, X_{2}\right)+\mathcal{H}\left(c_{6}^{\prime} X_{2}^{2}+c_{7}^{\prime} X_{1} X_{2}\right)+\mathcal{H}^{2}\left(c_{8}^{\prime} X_{1}+c_{9}^{\prime} X_{2}\right)+c_{10}^{\prime} \mathcal{H}^{3}
\end{aligned}
$$

for some $c_{6}^{\prime}, c_{7}^{\prime}, c_{8}^{\prime}, c_{9}^{\prime}, c_{10}^{\prime}$. Hence we can assume that the

$$
\mathcal{F}^{(3)}\left(X_{1}, X_{2}\right)+\mathcal{H} \mathcal{F}^{(2)}\left(X_{1}, X_{3}\right)=C_{3}\left(X_{1}, X_{2}\right)+c_{6} \mathcal{H} X_{2}^{2}+c_{7} \mathcal{H} X_{1} X_{2}
$$

using a matrix of the form $A=\left(\begin{array}{ccc}d^{2} & 0 & 0 \\ 0 & d & 0 \\ 0 & 0 & r\end{array}\right)$ we can further assume that $c_{6}, c_{7} \in\{0,1\}$. For the case of $c_{6}=c_{7}=0$ we obtain the following proposition:

Proposition 5.9. The stabilizer of the form

$$
\mathcal{F}^{(3)}\left(X_{1}, X_{2}\right)+\mathcal{H} \mathcal{F}^{(2)}\left(X_{1}, X_{3}\right)+O\left(\mathcal{H}^{2}\right)=C_{3}\left(X_{1}, X_{2}\right)+O\left(\mathcal{H}^{2}\right)
$$

is given by

$$
\operatorname{Stab}_{G}\left(C_{3}\left(X_{1}, X_{2}\right)+O\left(\mathcal{H}^{2}\right)\right)=\left\{\left(\begin{array}{lll}
d^{2} & 0 & 0 \\
\gamma & d & b \\
0 & 0 & c
\end{array}\right) \mid b, \gamma \in \mathbb{C}, d, c \in \mathbb{C}^{*}\right\} .
$$

For the case of $c_{6}=0, c_{7}=1$ we obtain the following proposition:

Proposition 5.10. The stabilizer of the form

$$
\mathcal{F}^{(3)}\left(X_{1}, X_{2}\right)+\mathcal{H} \mathcal{F}^{(2)}\left(X_{1}, X_{3}\right)+O\left(\mathcal{H}^{2}\right)=C_{3}\left(X_{1}, X_{2}\right)+\mathcal{H} X_{1} X_{2}+O\left(\mathcal{H}^{2}\right)
$$

is given by

$$
\operatorname{Stab}_{G}\left(C_{3}\left(X_{1}, X_{2}\right)+\mathcal{H} X_{1} X_{2}+O\left(\mathcal{H}^{2}\right)\right)=\left\{\left(\begin{array}{ccc}
d^{2} & 0 & a \\
-\frac{3 a}{d} & d & b \\
0 & 0 & d^{3}
\end{array}\right) \mid a, b \in \mathbb{C}, d \in \mathbb{C}^{*}\right\} .
$$

For the case of $c_{6}=1, c_{7}=0$ we obtain the following proposition:

Proposition 5.11. The stabilizer of the form

$$
\mathcal{F}^{(3)}\left(X_{1}, X_{2}\right)+\mathcal{H} \mathcal{F}^{(2)}\left(X_{1}, X_{3}\right)+O\left(\mathcal{H}^{2}\right)=C_{3}\left(X_{1}, X_{2}\right)+\mathcal{H} X_{2}^{2}+O\left(\mathcal{H}^{2}\right)
$$

is given by

$$
\operatorname{Stab}_{G}\left(C_{3}\left(X_{1}, X_{2}\right)+\mathcal{H} X_{2}^{2}+O\left(\mathcal{H}^{2}\right)\right)=\left\{\left(\begin{array}{ccc}
d^{2} & 0 & 0 \\
0 & d & b \\
0 & 0 & d^{4}
\end{array}\right) \mid b \in \mathbb{C}, d \in \mathbb{C}^{*}\right\} .
$$


For the case of $c_{6}=1, c_{7}=1$ we obtain the following proposition:

Proposition 5.12. The stabilizer of the form

$$
\mathcal{F}^{(3)}\left(X_{1}, X_{2}\right)+\mathcal{H} \mathcal{F}^{(2)}\left(X_{1}, X_{3}\right)=C_{3}\left(X_{1}, X_{2}\right)+\mathcal{H} X_{2}^{2}+\mathcal{H} X_{1} X_{2}+O\left(\mathcal{H}^{2}\right)
$$

is given by

$$
\begin{aligned}
\operatorname{Stab}_{G} & \left(C_{3}\left(X_{1}, X_{2}\right)+\mathcal{H} X_{1} X_{2}+\mathcal{H} X_{2}^{2}+O\left(\mathcal{H}^{2}\right)\right) \\
= & \left\{\left(\begin{array}{ccc}
d^{2} & 0 & \frac{d^{2}}{12}\left(d^{2}-1\right) \\
\frac{1}{2} d(1-d) & d & b \\
0 & 0 & d^{4}
\end{array}\right) \mid b \in \mathbb{C}, d \in \mathbb{C}^{*}\right\} .
\end{aligned}
$$

\subsection{1 $\mathcal{F}^{(3)}\left(X_{1}, X_{2}\right)=C_{3}\left(X_{1}, X_{2}\right), c_{6}=0$, and $c_{7}=0$}

Suppose that

$$
\begin{aligned}
\mathcal{R}^{2} & =\mathcal{F}^{(3)}\left(X_{1}, X_{2}\right)+\mathcal{H} \mathcal{F}^{(2)}\left(X_{1}, X_{2}\right)+\mathcal{H}^{2} \mathcal{F}^{(1)}\left(X_{1}, X_{2}\right)+c_{10} \mathcal{H}^{3} \\
& =C_{3}\left(X_{1}, X_{2}\right)+\mathcal{H}^{2}\left(c_{8} X_{1}+c_{9} X_{2}\right)+c_{10} \mathcal{H}^{3}
\end{aligned}
$$

$$
\begin{aligned}
& \text { Acting with } A=\left(\begin{array}{ccc}
d^{2} & 0 & 0 \\
\gamma & d & b \\
0 & 0 & c
\end{array}\right)^{-1} \in \operatorname{Stab}_{G}\left(C_{3}\left(X_{1}, X_{2}\right)+O\left(\mathcal{H}^{2}\right)\right) \text { on } \mathcal{R}^{2} \text { we have } \\
& \qquad \begin{array}{l}
\mathcal{R}^{2}=C_{3}\left(X_{1}, X_{2}\right)+\mathcal{H}^{2}\left(c_{8} X_{1}+c_{9} X_{2}\right)+c_{10} \mathcal{H}^{3} \\
\longmapsto C_{3}\left(X_{1}, X_{2}\right)+\mathcal{H}^{2}\left(c_{8}^{\prime} X_{1}+c_{9}^{\prime} X_{2}\right)+c_{10}^{\prime} \mathcal{H}^{3},
\end{array}
\end{aligned}
$$

where $c_{8}^{\prime}=c^{2}\left(d^{-4} c_{8}+d^{-6} \gamma c_{9}\right), c_{9}^{\prime}=c^{2} d^{-5} c_{9}, c_{10}^{\prime}=d^{-6}\left(c^{2} b c_{9}+c^{3} c_{10}\right)$. If $c_{9}=0$ and $c_{8} \neq 0$ we define the canonical form to be with $c_{8}=1$ and $c_{10}=r e^{i \theta}$ with $r \geq 0$ and $\theta \in[0, \pi)$. If $c_{9}=0$ and $c_{8}=0$ we define the canonical form to be with $c_{10} \in\{0,1\}$. If $c_{9} \neq 0$ then the canonical form is given by $\mathcal{R}^{2}=C_{3}\left(X_{1}, X_{2}\right)+\mathcal{H}^{2} X_{2}$.

\subsection{2 $\mathcal{F}^{(3)}\left(X_{1}, X_{2}\right)=C_{3}\left(X_{1}, X_{2}\right), c_{6}=0$, and $c_{7}=1$}

Suppose that

$$
\begin{aligned}
& \qquad \begin{aligned}
\mathcal{R}^{2} & =\mathcal{F}^{(3)}\left(X_{1}, X_{2}\right)+\mathcal{H} \mathcal{F}^{(2)}\left(X_{1}, X_{2}\right)+\mathcal{H}^{2} \mathcal{F}^{(1)}\left(X_{1}, X_{2}\right)+c_{10} \mathcal{H}^{3} \\
& =C_{3}\left(X_{1}, X_{2}\right)+\mathcal{H} X_{1} X_{2}+\mathcal{H}^{2}\left(c_{8} X_{1}+c_{9} X_{2}\right)+c_{10} \mathcal{H}^{3} .
\end{aligned} \\
& \text { Acting with } A=\left(\begin{array}{ccc}
d^{2} & 0 & a \\
-\frac{3 a}{d} & d & b \\
0 & 0 & d^{3}
\end{array}\right) \quad \operatorname{Stab}_{G}\left(C_{3}\left(X_{1}, X_{2}\right)+\mathcal{H} X_{1} X_{2}+O\left(\mathcal{H}^{2}\right)\right) \text { on } \mathcal{R}^{2} \text { we have } \\
& \mathcal{R}^{2}=C_{3}\left(X_{1}, X_{2}\right)+\mathcal{H} X_{1} X_{2}+\mathcal{H}^{2}\left(c_{8} X_{1}+c_{9} X_{2}\right)+c_{10} \mathcal{H}^{3} \\
& \\
& \longmapsto C_{3}\left(X_{1}, X_{2}\right)+\mathcal{H} X_{1} X_{2}+\mathcal{H}^{2}\left(c_{8}^{\prime} X_{1}+c_{9}^{\prime} X_{2}\right)+c_{10}^{\prime} \mathcal{H}^{3},
\end{aligned}
$$

where $c_{8}^{\prime}=\frac{b}{d}+d^{2} c_{8}-3 \frac{a}{d} c_{9}, c_{9}^{\prime}=\frac{a}{d^{2}}+c_{9} d, c_{10}^{\prime}=\frac{a^{3}}{d^{6}}+\frac{a b}{d^{3}}+a c_{8}+b c_{9}+d^{3} c_{10}$. Hence we can always arrange that $c_{8}=c_{9}=0$ and $c_{10} \in\{0,1\}$ and this will be the canonical form in this case. 


\subsection{3 $\mathcal{F}^{(3)}\left(X_{1}, X_{2}\right)=C_{3}\left(X_{1}, X_{2}\right), c_{6}=1$, and $c_{7}=0$}

Suppose that

$$
\begin{aligned}
\mathcal{R}^{2} & =\mathcal{F}^{(3)}\left(X_{1}, X_{2}\right)+\mathcal{H} \mathcal{F}^{(2)}\left(X_{1}, X_{2}\right)+\mathcal{H}^{2} \mathcal{F}^{(1)}\left(X_{1}, X_{2}\right)+c_{10} \mathcal{H}^{3} \\
& =C_{3}\left(X_{1}, X_{2}\right)+\mathcal{H} X_{2}^{2}+\mathcal{H}^{2}\left(c_{8} X_{1}+c_{9} X_{2}\right)+c_{10} \mathcal{H}^{3} .
\end{aligned}
$$

Acting with $A=\left(\begin{array}{ccc}d^{2} & 0 & 0 \\ 0 & d & b \\ 0 & 0 & d^{4}\end{array}\right)^{-1} \in \operatorname{Stab}_{G}\left(C_{3}\left(X_{1}, X_{2}\right)+\mathcal{H} X_{2}^{2}+O\left(\mathcal{H}^{2}\right)\right)$ on $\mathcal{R}^{2}$ we have

$$
\begin{aligned}
\mathcal{R}^{2} & =C_{3}\left(X_{1}, X_{2}\right)+\mathcal{H} X_{2}^{2}+\mathcal{H}^{2}\left(c_{8} X_{1}+c_{9} X_{2}\right)+c_{10} \mathcal{H}^{3} \\
& \longmapsto C_{3}\left(X_{1}, X_{2}\right)+\mathcal{H} X_{2}^{2}+\mathcal{H}^{2}\left(c_{8}^{\prime} X_{1}+c_{9}^{\prime} X_{2}\right)+c_{10}^{\prime} \mathcal{H}^{3},
\end{aligned}
$$

where $c_{8}^{\prime}=d^{4} c_{8}, c_{9}^{\prime}=2 \frac{b}{d}+c_{9} d^{3}, c_{10}^{\prime}=\frac{b^{2}}{d^{2}}+d^{2} b c_{9}+d^{6} c_{10}$. Hence we can always arrange that $c_{9}=0$ and either $c_{8}=0$ and $c_{10} \in\{0,1\}$ or $c_{8}=1$ and $c_{10}=r e^{i \theta}$ with $r \geq 0$ and $\theta \in\left[0, \frac{\pi}{2}\right)$.

\subsection{4 $\mathcal{F}^{(3)}\left(X_{1}, X_{2}\right)=C_{3}\left(X_{1}, X_{2}\right), c_{6}=1$, and $c_{7}=1$}

Suppose that

$$
\begin{aligned}
\mathcal{R}^{2} & =\mathcal{F}^{(3)}\left(X_{1}, X_{2}\right)+\mathcal{H} \mathcal{F}^{(2)}\left(X_{1}, X_{2}\right)+\mathcal{H}^{2} \mathcal{F}^{(1)}\left(X_{1}, X_{2}\right)+c_{10} \mathcal{H}^{3} \\
& =C_{3}\left(X_{1}, X_{2}\right)+\mathcal{H} X_{1} X_{2}+\mathcal{H} X_{2}^{2}+\mathcal{H}^{2}\left(c_{8} X_{1}+c_{9} X_{2}\right)+c_{10} \mathcal{H}^{3}
\end{aligned}
$$

Acting with $A=\left(\begin{array}{ccc}d^{2} & 0 & \frac{d^{2}}{12}\left(d^{2}-1\right) \\ \frac{1}{2} d(1-d) & d & b \\ 0 & 0 & d^{4}\end{array}\right)^{-1} \in \operatorname{Stab}_{G}\left(C_{3}\left(X_{1}, X_{2}\right)+\mathcal{H} X_{1} X_{2}+\mathcal{H} X_{2}^{2}+\right.$ $\left.O\left(\mathcal{H}^{2}\right)\right)$ on $\mathcal{R}^{2}$ we have

$$
\begin{aligned}
\mathcal{R}^{2} & =C_{3}\left(X_{1}, X_{2}\right)+\mathcal{H} X_{1} X_{2}+\mathcal{H} X_{2}^{2}+\mathcal{H}^{2}\left(c_{8} X_{1}+c_{9} X_{2}\right)+c_{10} \mathcal{H}^{3} \\
& \longmapsto C_{3}\left(X_{1}, X_{2}\right)+\mathcal{H} X_{1} X_{2}+\mathcal{H} X_{2}^{2}+\mathcal{H}^{2}\left(c_{8}^{\prime} X_{1}+c_{9}^{\prime} X_{2}\right)+c_{10}^{\prime} \mathcal{H}^{3},
\end{aligned}
$$

where $c_{8}^{\prime}=\frac{b}{d}-\frac{1}{48}\left(d^{2}-1\right)(d-1)^{2}+d^{4} c_{8}+\frac{1}{2} d^{3}(1-d) c_{9}, c_{9}^{\prime}=\frac{1}{12} d^{3}\left(d^{2}-1\right)+2 \frac{b}{d}+c_{9} d^{3}$, $c_{10}^{\prime}=\frac{1}{12^{3}}\left(d^{2}-1\right)^{3}+\frac{1}{12}\left(d^{2}-1\right) b+\frac{b^{2}}{d^{2}}+\frac{1}{12} d^{4}\left(d^{2}-1\right) c_{8}+d^{2} b c_{9}+d^{6} c_{10}$. Hence we can assume that $c_{9}=c_{8}=0$ and the canonical form is given by

$$
\mathcal{R}^{2}=X_{1}^{3}+\mathcal{H} X_{1} X_{2}+\mathcal{H} X_{2}^{2}+c_{10} \mathcal{H}^{3}
$$

with $c_{10} \in \mathbb{C}$.

\subsection{Fourth case: $\widetilde{\mathcal{F}}^{(3)}=0$}

A similar (but simpler) calculation to the one that was done in the previous section leads to the possibilities for the canonical forms for $\mathcal{F} \in \mathbb{C}^{[3]}\left[x_{1}, x_{2}, x_{3}\right]$ with a vanishing $\mathcal{F}^{(3)}$. For example it easy to show the following lemma.

Lemma 5.13. Given $\mathcal{F} \in \mathbb{C}^{[3]}\left[x_{1}, x_{2}, x_{3}\right]$ with a vanishing $\mathcal{F}^{(3)}$ we can find an explicit matrix $A \in G$ such that the $\mathcal{F}^{(2)}$ part of $A \cdot \mathcal{F}$ is equal to exactly one of the following three cases: $X_{1}^{2}$, $X_{1} X_{2}, 0$. 
Table 1. List of canonical forms of $\mathcal{R}^{2}$ for the nondegenerate free quadratic algebras.

\begin{tabular}{|l|l|l|}
\hline \multicolumn{2}{|c|}{ Canonical forms of $\mathcal{R}^{2}$ for the nondegenerate free quadratic algebras } & \\
\hline & $\mathcal{R}^{2}$ & domain of parameters \\
\hline 1a & $X_{1} X_{2}\left(X_{1}+X_{2}\right)+c_{8} X_{1} \mathcal{H}^{2}+c_{9} X_{2} \mathcal{H}^{2}+\mathcal{H}^{3}$ & $c_{8}, c_{9} \in \mathbb{C}$, see remark below \\
\hline 1b & $X_{1} X_{2}\left(X_{1}+X_{2}\right)+X_{1} \mathcal{H}^{2}+c_{9} X_{2} \mathcal{H}^{2}$ & $c_{9} \in \mathbb{C}$, see remark below \\
\hline 1c & $X_{1} X_{2}\left(X_{1}+X_{2}\right)$ & \\
\hline 1d & $X_{1} X_{2}\left(X_{1}+X_{2}\right)+\mathcal{H}_{1} X_{2}+c_{8} X_{1} \mathcal{H}^{2}+c_{9} X_{2} \mathcal{H}^{2}+c_{10} \mathcal{H}^{3}$ & $c_{8}, c_{9}, c_{10} \in \mathbb{C}$ \\
\hline 2a & $X_{1}^{2} X_{2}+X_{1} \mathcal{H}^{2}+X_{2} \mathcal{H}^{2}+c_{10} \mathcal{H}^{3}$ & $c_{10} \in \mathbb{C}$ \\
\hline 2b & $X_{1}^{2} X_{2}+c_{9} X_{2} \mathcal{H}^{2}+c_{10} \mathcal{H}^{3}$ & $c_{9}, c_{10} \in\{0,1\}$ \\
\hline 2c & $X_{1}^{2} X_{2}+\mathcal{H} X_{2}^{2}+X_{1} \mathcal{H}^{2}+c_{9} X_{2} \mathcal{H}^{2}+c_{10} \mathcal{H}^{3}$ & $c_{9}, c_{10} \in \mathbb{C}$ \\
\hline 2d & $X_{1}^{2} X_{2}+\mathcal{H} X_{2}^{2}+X_{2} \mathcal{H}^{2}+c_{10} \mathcal{H}^{3}$ & $c_{10} \in \mathbb{C}$ \\
\hline 2e & $X_{1}^{2} X_{2}+\mathcal{H} X_{2}^{2}+c_{10} \mathcal{H}^{3}$ & $c_{10} \in\{0,1\}$ \\
\hline 3a & $X_{1}^{3}+X_{1} \mathcal{H}^{2}+c_{10} \mathcal{H}^{3}$ & $c_{10} \in \mathbb{C}$ \\
\hline 3b & $X_{1}^{3}+\mathcal{H}^{3}$ & \\
\hline 3c & $X_{1}^{3}+X_{2} \mathcal{H}^{2}$ & $c_{10} \in\{0,1\}$ \\
\hline 3d & $X_{1}^{3}+\mathcal{H} X_{1} X_{2}+c_{10} \mathcal{H}^{3}$ & $c_{10} \in\{0,1\}$ \\
\hline 3e & $X_{1}^{3}+\mathcal{H} X_{2}^{2}+c_{10} \mathcal{H}^{3}$ & $r \geq 0, \theta \in\left[0, \frac{\pi}{2}\right)$ \\
\hline 3f & $X_{1}^{3}+\mathcal{H} X_{2}^{2}+X_{1} \mathcal{H}^{2}+r e^{i \theta} \mathcal{H}^{3}$ & $c_{10} \in \mathbb{C}$ \\
\hline 3g & $X_{1}^{3}+\mathcal{H} X_{1} X_{2}+\mathcal{H} X_{2}^{2}+c_{10} \mathcal{H}^{3}$ & \\
\hline 4a & $\mathcal{H} X_{1}^{2}+\mathcal{H}^{2} X_{2}$ & $c_{10} \in \mathbb{C}$ \\
\hline 4b & $\mathcal{H} X_{1}^{2}+\mathcal{H}^{2} X_{1}+c_{10} \mathcal{H}^{3}$ & $c_{10} \in\{0,1\}$ \\
\hline 4c & $\mathcal{H} X_{1}^{2}+c_{10} \mathcal{H}^{3}$ & $c_{10} \in \mathbb{C}$ \\
\hline 4d & $\mathcal{H} X_{1} X_{2}+\mathcal{H}^{2}\left(X_{1}+X_{2}\right)+c_{10} \mathcal{H}^{3}$ & $c_{8}, c_{10} \in\{0,1\}$ \\
\hline 4e & $\mathcal{H} X_{1} X_{2}+c_{8} \mathcal{H}^{2} X_{1}+c_{10} \mathcal{H}^{3}$ & $c_{10} \in\{0,1\}$ \\
\hline 4f & $\mathcal{H}^{2} X_{1}$ & \\
\hline 4g & $c_{10} \mathcal{H}^{3}$ & \\
\hline & & \\
\hline
\end{tabular}

Remark 5.14. For each value of the parameter in the first two lines of Table 1 if

$$
c_{8}^{\prime}=c^{2}\left(\alpha c_{8}+\gamma c_{9}\right), \quad c_{9}^{\prime}=c^{2}\left(\beta c_{8}+\delta c_{9}\right), \quad c_{10}^{\prime}=c^{3} c_{10},
$$

for $c \in \mathbb{C}^{*}$ and

$$
\begin{aligned}
\left(\begin{array}{ll}
\alpha & \beta \\
\gamma & \delta
\end{array}\right) \in \Omega\left(C_{1}\right)= & \left\{\left(\begin{array}{ll}
0 & 1 \\
1 & 0
\end{array}\right),\left(\begin{array}{cc}
0 & 1 \\
-1 & -1
\end{array}\right),\left(\begin{array}{ll}
1 & 0 \\
0 & 1
\end{array}\right)\right\} \\
& \coprod\left\{\left(\begin{array}{cc}
-1 & -1 \\
0 & -1
\end{array}\right),\left(\begin{array}{cc}
1 & 0 \\
-1 & -1
\end{array}\right),\left(\begin{array}{cc}
-1 & -1 \\
1 & 0
\end{array}\right)\right\}
\end{aligned}
$$

then the system with parameters $c_{8}, c_{9}, c_{10}$ isomorphic to the one with $c_{8}^{\prime}, c_{9}^{\prime}, c_{10}^{\prime}$.

\subsection{Comparison of geometric and abstract nondegenerate quadratic algebras}

There is a close relationship between the canonical forms of abstract quadratic algebras and Stäckel equivalence classes of nondegenerate superintegrable systems. To demonstrate this we treat one example in detail. The superintegrable system $S 9$, with nondegenerate potential, can be defined by

$$
\begin{aligned}
\mathcal{R}^{2}= & \mathcal{L}_{1}^{2} \mathcal{L}_{2}+\mathcal{L}_{1} \mathcal{L}_{2}^{2}+\mathcal{L}_{1} \mathcal{L}_{2}\left(\mathcal{H}-a_{4}\right)-a_{2}\left(\mathcal{H}-a_{4}\right)^{2}-2 a_{2} \mathcal{L}_{1}\left(\mathcal{H}-a_{4}\right) \\
& -2 a_{2} \mathcal{L}_{2}\left(\mathcal{H}-a_{4}\right)-\left(a_{3}+a_{2}\right) \mathcal{L}_{1}^{2}-\left(a_{3}+3 a_{2}+a_{1}\right) \mathcal{L}_{1} \mathcal{L}_{2}-\left(a_{2}+a_{1}\right) \mathcal{L}_{2}^{2} \\
& +\left(2 a_{2} a_{3}+2 a_{2}^{2}+2 a_{1} a_{2}\right)\left(\mathcal{H}-a_{4}\right)+2\left(a_{2}^{2}+a_{2} a_{3}+a_{1} a_{2}\right) \mathcal{L}_{1} \\
& +2\left(a_{2}^{2}+a_{2} a_{3}+a_{1} a_{2}\right) \mathcal{L}_{2}+2 a_{1} a_{2} a_{3}-2 a_{1} a_{2}^{2}-2 a_{2}^{2} a_{3}-a_{2} a_{3}^{2}-a_{2} a_{1}^{2}-a_{2}^{3},
\end{aligned}
$$


where the $a_{j}$ are the parameters in the potential. To perform a general Stäckel transform of this system with nonsingular transform matrix $\left.C=\left(c_{j k}\right): 1\right)$ we set $a_{j}=\sum_{k=1}^{4} c_{j k} b_{k}, k=1, \ldots, 4$ where the $b_{k}$ are the new parameters, 2) we make the replacements $\mathcal{H} \rightarrow-b_{4}, b_{4} \rightarrow-\mathcal{H}$ and 3 ) we then set all parameters $b_{j}=0$ to determine the free quadratic algebra. The result is

$$
\begin{aligned}
\mathcal{R}^{2}= & c_{24}\left(c_{14}^{2}+2 c_{14} c_{24}-2 c_{14} c_{34}+2 c_{14} c_{44}+c_{24}^{2}+2 c_{24} c_{34}+2 c_{24} c_{44}+c_{34}^{2}+2 c_{34} c_{44}\right. \\
& \left.+c_{44}^{2}\right) \mathcal{H}^{3}+\left(2 c_{24}\left(c_{14}+c_{24}+c_{34}+c_{44}\right) \mathcal{L}_{1}+2 c_{24}\left(c_{14}+c_{24}+c_{34}+c_{44}\right) \mathcal{L}_{2}\right) \mathcal{H}^{2} \\
& +\left(c_{24}+c_{34}\right) \mathcal{L}_{1}^{2} \mathcal{H}+\left(c_{14}+c_{24}\right) \mathcal{L}_{2}^{2} \mathcal{H}+\left(c_{14}+3 c_{24}+c_{34}+c_{44}\right) \mathcal{L}_{2} \mathcal{L}_{1} \mathcal{H} \\
& +\mathcal{L}_{1}^{2} \mathcal{L}_{2}+\mathcal{L}_{1} \mathcal{L}_{2}^{2} .
\end{aligned}
$$

We put this in canonical form by making the choices $\mathcal{L}_{1}=X_{1}+\left(c_{24}+c_{14}\right) \mathcal{H}, \mathcal{L}_{2}=X_{2}+\left(c_{34}+\right.$ $\left.c_{24}\right) \mathcal{H}$. The final result is

$$
\text { [1111]: } \quad \mathcal{R}^{2}=X_{1}^{2} X_{2}+X_{1} X_{2}^{2}+A_{1} X_{1} \mathcal{H}^{2}+A_{2} X_{2} \mathcal{H}^{2}+A_{3} X_{1} X_{2} \mathcal{H}+A_{4} \mathcal{H}^{3}
$$

where

$$
\begin{array}{ll}
A_{1}=\left(c_{24}-c_{34}\right)\left(c_{14}+c_{44}\right), & A_{2}=\left(c_{34}+c_{44}\right)\left(c_{24}-c_{14}\right), \\
A_{3}=-c_{14}-c_{24}-c_{34}+c_{44}, & A_{4}=\left(c_{14}-c_{24}+c_{34}+c_{44}\right)\left(c_{14} c_{34}+c_{24} c_{44}\right) .
\end{array}
$$

The possible canonical forms in Table 1 associated with the equivalence class [1111] depend on the possible choices of $c_{i j}$ with $\operatorname{det} C \neq 0$. The possible canonical forms are $1 a, 1 b, 1 d$ all cases.

The superintegrable system $E 1$, with nondegenerate potential, can be defined by

$$
\begin{aligned}
\mathcal{R}^{2}= & \mathcal{L}_{1} \mathcal{L}_{2}\left(\mathcal{H}-a_{4}\right)+\mathcal{L}_{2}^{2} \mathcal{L}_{1}-a_{3}\left(\mathcal{H}-a_{4}\right)^{2}-2 a_{3} \mathcal{L}_{2}\left(\mathcal{H}-a_{4}\right) \\
& -\left(a_{3}+a_{2}\right) \mathcal{L}_{2}^{2}-a_{1} \mathcal{L}_{1}^{2}+4 a_{1} a_{2} a_{3} .
\end{aligned}
$$

Going through the same procedure as above, we obtain the equivalence class

$$
\begin{aligned}
{[211]: \quad \mathcal{R}^{2}=} & -X_{2} X_{1}^{2}+\left(2 c_{14} c_{24}+2 c_{14} c_{34}+\frac{1}{4} c_{44}^{2}\right) X_{2} \mathcal{H}^{2}+c_{44}\left(-c_{34}+c_{24}\right) X_{1} \mathcal{H}^{2} \\
& +c_{14} X_{2}^{2} \mathcal{H}\left(-2 c_{14} c_{24} c_{34}+c_{14} c_{24}^{2}+c_{14} c_{34}^{2}+\frac{1}{2} c_{44}^{2} c_{24}+\frac{1}{2} c_{34} c_{44}^{2}\right) \mathcal{H}^{3}
\end{aligned}
$$

The canonical forms associated with this equivalence class are $2 a, 2 b, 2 c, 2 d, 2 e$, all cases.

The superintegrable system $E 8$, with nondegenerate potential, can be defined by

$$
\mathcal{R}^{2}=\mathcal{L}_{2}^{2} \mathcal{L}_{1}-a_{2}\left(\mathcal{H}-a_{4}\right) \mathcal{L}_{2}+4 a_{1} a_{3} \mathcal{L}_{1}+a_{1}\left(\mathcal{H}-a_{4}\right)^{2}-a_{3} a_{2}^{2} .
$$

The equivalence class is

$$
[22]: \quad \mathcal{R}^{2}=X_{1}^{2} X_{2}-c_{24} c_{44} X_{1} \mathcal{H}^{2}+4 c_{14} c_{34} X_{2} \mathcal{H}^{2}+\left(-c_{14} c_{44}^{2}+c_{34} c_{24}^{2}\right) \mathcal{H}^{3} .
$$

The canonical form associated with this equivalence class is $2 a$ : all cases.

The superintegrable system $E 2$ can be defined by

$$
\begin{aligned}
\mathcal{R}^{2}= & \mathcal{L}_{1}^{3}+\mathcal{L}_{1} \mathcal{H}^{2}-2 \mathcal{L}_{1}^{2} \mathcal{H}+\left(-2 a_{4} \mathcal{L}_{1}-a_{2} \mathcal{L}_{2}\right) \mathcal{H}+2 a_{4} \mathcal{L}_{1}^{2}+\left(a_{2} \mathcal{L}_{2}+4 a_{1} a_{3}+a_{4}^{2}\right) \mathcal{L}_{1} \\
& +4 a_{1} \mathcal{L}_{2}^{2}+a_{2} a_{4} \mathcal{L}_{2}-\frac{1}{4} a_{2}^{2} a_{3} .
\end{aligned}
$$

The equivalence class is

$$
\begin{aligned}
{[31]: \quad \mathcal{R}^{2}=} & X_{1}^{3}+\left(c_{14} X_{1} X_{2}-4 c_{34} X_{2}^{2}+c_{44} X_{1}^{2}\right) \mathcal{H}+4 c_{34} c_{24} X_{1} \mathcal{H}^{2} \\
& +\frac{1}{4} c_{24}\left(c_{14}^{2}+16 c_{34} c_{44}\right) \mathcal{H}^{3}
\end{aligned}
$$


The canonical forms associated with this equivalence class are $3 d$ : all cases, $3 e: c_{10}=0,3 f$ : all cases, $3 g: c_{10}=0$.

The superintegrable system $E 10$ can be defined by

$$
\begin{aligned}
\mathcal{R}^{2}= & \mathcal{L}_{1}^{3}+2 a_{1} \mathcal{L}_{1}^{2}-a_{3} \mathcal{L}_{1} \mathcal{L}_{2}+a_{3}\left(\mathcal{H}-a_{4}\right)^{2}+2 a_{2} \mathcal{L}_{1}\left(\mathcal{H}-a_{4}\right) \\
& +2 a_{1} a_{2}\left(\mathcal{H}-a_{4}\right)+a_{1}^{2} \mathcal{L}_{1}+a_{2}^{2} \mathcal{L}_{2}
\end{aligned}
$$

The equivalence class contains

$$
\begin{aligned}
{[4]: \quad \mathcal{R}^{2}=} & X_{1}^{3}+c_{34} X_{1} X_{2} \mathcal{H}+\left(c_{24}^{2}+\frac{2}{3} c_{14} c_{34}\right) X_{2} \mathcal{H}^{2} \\
& +\frac{1}{27} \frac{\left(8 c_{14}^{3} c_{34}+9 c_{14}^{2} c_{24}^{2}+54 c_{14} c_{24} c_{34} c_{44}+54 c_{24}^{3} c_{44}-27 c_{34}^{2} c_{44}^{2}\right)}{c_{34}} \mathcal{H}^{3},
\end{aligned}
$$

if $c_{34} \neq 0$. If $c_{34}=0, c_{24} \neq 0$ it contains

$$
[4]^{\prime}: \quad \mathcal{R}^{2}=X_{1}^{3}-2 c_{14}^{2} X_{1}^{2} \mathcal{H}+c_{24}^{2} X_{2} \mathcal{H}^{2}+2 c_{14} c_{24} c_{44} \mathcal{H}^{3}
$$

and if $c_{34}=c_{24}=0$ it contains

$$
[4]^{\prime \prime}: \quad \mathcal{R}^{2}=X_{1}^{3}+c_{14}^{2} X_{1} \mathcal{H}^{2}-2 c_{14} X_{1}^{2} \mathcal{H} .
$$

The canonical form associated with [4] is $3 d$ all cases. The canonical form associated with [4] is $3 c$ : all cases, and the canonical form associated with $[4]^{\prime \prime}$ is $3 a: c_{10} \neq 0$.

The superintegrable system $E 3^{\prime}$ can be defined by

$$
\mathcal{R}^{2}=-4 a_{1}\left(\mathcal{L}_{1}^{2}+\mathcal{L}_{2}^{2}-\mathcal{L}_{2} \mathcal{H}\right)-2 a_{2} a_{3} \mathcal{L}_{1}+\left(a_{2}^{2}-a_{3}^{2}-4 a_{1} a_{4}\right) \mathcal{L}_{2}-a_{3}^{2} a_{4}+a_{3}^{2} \mathcal{H}
$$

The canonical form is

$$
[0]: \quad \mathcal{R}^{2}=4 c_{14}\left(X_{1}^{2}+X_{2}^{2}\right) \mathcal{H}-\frac{\left(4 c_{14} c_{44}-c_{24}^{2}-c_{34}^{2}\right)^{2}}{16 c_{14}} \mathcal{H}^{3},
$$

if $c_{14} \neq 0$; if $c_{14}=0$ it is

$$
[0]^{\prime}: \quad \mathcal{R}^{2}=-2 c_{24} c_{34} X_{1} \mathcal{H}^{2}+\left(c_{24}^{2}-c_{34}^{2}\right) X_{2} \mathcal{H}^{2}+c_{34}^{2} c_{44} \mathcal{H}^{3} .
$$

The canonical forms associated with [0] are $4 d$ : all cases, $4 e$ : all cases, and the canonical forms associated with $[0]^{\prime}$ are $4 f$ : all cases.

Heisenberg systems. In addition there are systems that can be obtained from the geometric systems above by contractions from $\mathfrak{s o}(4, \mathbb{C})$ to $\mathfrak{e}(3, \mathbb{C})$. These are not Bôcher contractions and the contracted systems are not superintegrable, because the Hamiltonians become singular. However, they do form quadratic algebras and many have the interpretation of time-dependent Schrödinger equations in 2D spacetime, so we also consider them geometrical. Some of these were classified in [23] where they were called Heisenberg systems since they appeared in quadratic algebras formed from 2 nd order elements in the Heisenberg algebra with generators $\mathcal{M}_{1}=p_{x}$, $\mathcal{M}_{2}=x p_{y}, \mathcal{E}=p_{y}$, where $\mathcal{E}^{2}=\mathcal{H}$. The systems are all of type 4 . We will devote a future paper to their study. The ones classified so far are $4 a$ : all cases, $4 c$ : $c_{10}=0,4 e: c_{10}=0,4 f:$ all cases, $4 g$ : all cases.

All these results relating geometric systems to abstract systems are summarized in Table 2 . 
Table 2. Matching of geometric with abstract quadratic algebras.

\begin{tabular}{|l|l|l|l|}
\hline Class & \multicolumn{3}{|c|}{ Canonical form } \\
\hline 1 & $a:$ all cases & $b:$ all cases & $c:$ no \\
\hline 1 & $d:$ all cases & & \\
\hline 2 & $a:$ all cases & $b$ all cases & $c:$ all cases \\
\hline 2 & $d:$ all cases & $e:$ all cases & \\
\hline 3 & $a: c_{10} \neq 0$ & $b:$ no & $c:$ all cases \\
\hline 3 & $d:$ all cases & $e: c_{10}=0$ & $f:$ all cases \\
\hline 3 & $g: c_{10}=0$ & & \\
\hline 4 & $a:$ all cases & $b:$ no & $c: c_{10}=0$ \\
\hline 4 & $d:$ all cases & $e:$ all cases & $f:$ all cases \\
\hline 4 & $4 g:$ all cases & & \\
\hline
\end{tabular}

\section{The quadratic algebras of the free $2 \mathrm{D}$ second order superintegrable systems}

In this section we list all canonical forms of the Casimirs of the quadratic algebras of free nondegenerate 2D superintegrable systems on a constant curvature space or a Darboux space. We list the canonical forms arising from superintegrable systems on a constant curvature spaces in Table 3 and those arising from superintegrable systems on a Darboux space in Table 4. In the next section we study contractions between these quadratic algebras.

Table 3. Canonical forms of the Casimirs of quadratic algebras of free nondegenerate 2D superintegrable systems that lie inside $\mathcal{U}(\mathfrak{s o}(3, \mathbb{C}))$ and $\mathcal{U}(\mathfrak{e}(2, \mathbb{C}))$.

\begin{tabular}{|l|l|}
\hline System & Canonical forms of $\mathcal{R}^{2}$ \\
\hline$\widetilde{E}_{17}$ & $\mathcal{L}_{1}^{2} \mathcal{L}_{2}$ \\
\hline$\widetilde{E}_{16}$ & $\mathcal{L}_{1}^{2} \mathcal{L}_{2}+\mathcal{H} \mathcal{L}_{2}^{2}$ \\
\hline$\widetilde{E}_{1}$ & $\mathcal{L}_{1}^{2} \mathcal{L}_{2}+\mathcal{H}^{2} \mathcal{L}_{2}$ \\
\hline$\widetilde{E}_{8}$ & $\mathcal{L}_{1}^{2} \mathcal{L}_{2}$ \\
\hline$\widetilde{E}_{3}^{\prime}$ & 0 \\
\hline$\widetilde{E}_{2}$ & $\mathcal{L}_{1}^{3}+\mathcal{H}^{2} \mathcal{L}_{1}+\frac{2 i}{3 \sqrt{3}} \mathcal{H}^{3}$ \\
\hline$\widetilde{E}_{7}$ & $\mathcal{L}_{1}^{2} \mathcal{L}_{2}, \forall a$ \\
\hline$\widetilde{E}_{9}$ & $\mathcal{L}_{1}^{3}+\mathcal{H}^{2} \mathcal{L}_{1}+\frac{2 i}{3 \sqrt{3}} \mathcal{H}^{3}$ \\
\hline$\widetilde{E}_{11}$ & $\mathcal{H}^{2} \mathcal{L}_{1}$ \\
\hline$\widetilde{E}_{10}$ & $\mathcal{L}_{1}^{3}$ \\
\hline$\widetilde{E}_{15}$ & $\mathcal{L}_{1}^{3}$ \\
\hline$\widetilde{E}_{20}$ & $\mathcal{H L}_{1} \mathcal{L}_{2}$ \\
\hline$\widetilde{E}_{19}$ & $\mathcal{L}_{1}^{2} \mathcal{L}_{2}+\mathcal{H}^{2} \mathcal{L}_{2}$ \\
\hline$\widetilde{S}_{9}$ & $\mathcal{L}_{1} \mathcal{L}_{2}\left(\mathcal{L}_{1}+\mathcal{L}_{2}\right)+\mathcal{H} \mathcal{L}_{1} \mathcal{L}_{2}$ \\
\hline$\widetilde{S}_{4}$ & $\mathcal{L}_{1}^{2} \mathcal{L}_{2}$ \\
\hline$\widetilde{S}_{7}$ & $\mathcal{L}_{1} \mathcal{L}_{2}\left(\mathcal{L}_{1}+\mathcal{L}_{2}\right)+\mathcal{H} \mathcal{L}_{1} \mathcal{L}_{2}-\frac{1}{4} \mathcal{H}^{2} \mathcal{L}_{1}-\frac{1}{4} \mathcal{H}^{2} \mathcal{L}_{2}-\frac{1}{4} \mathcal{H}^{3}$ \\
\hline$\widetilde{S}_{8}$ & $\mathcal{L}_{1} \mathcal{L}_{2}\left(\mathcal{L}_{1}+\mathcal{L}_{2}\right)+\mathcal{H} \mathcal{L}_{1} \mathcal{L}_{2}$ \\
\hline$\widetilde{S}_{2}$ & $\mathcal{L}_{1}^{2} \mathcal{L}_{2}$ \\
\hline$\widetilde{S}_{1}$ & $\mathcal{L}_{1}^{3}$ \\
\hline
\end{tabular}


Table 4. Canonical forms of the Casimirs of quadratic algebras of free nondegenerate 2D Darboux superintegrable systems.

\begin{tabular}{|l|l|}
\hline System & Canonical forms of $\mathcal{R}^{2}$ \\
\hline$\widetilde{D} 1 A, b=0$ & $\mathcal{L}_{1}^{3}+\mathcal{H} \mathcal{L}_{1} \mathcal{L}_{2}$ \\
\hline$\widetilde{D} 1 A, b \neq 0$ & $\mathcal{L}_{1}^{3}+\mathcal{H} \mathcal{L}_{1} \mathcal{L}_{2}+\mathcal{H}^{3}$ \\
\hline$\widetilde{D} 1 B$ & $\mathcal{L}_{1}^{3}+\mathcal{H} \mathcal{L}_{1} \mathcal{L}_{2}$ \\
\hline$\widetilde{D} 1 C$ & $\mathcal{H}^{2} \mathcal{L}_{1}$ \\
\hline$\widetilde{D} 2 A$ & $\mathcal{L}_{1}^{3}+\mathcal{H}^{2} \mathcal{L}_{1}+\frac{2 i}{3 \sqrt{3}} \mathcal{H}^{3}$ \\
\hline$\widetilde{D} 2 B$ & $\mathcal{L}_{1}^{2} \mathcal{L}_{2}+\mathcal{H}^{2} \mathcal{L}_{1}+\mathcal{H}^{2} \mathcal{L}_{2}+i \mathcal{H}^{3}$ \\
\hline$\widetilde{D} 2 C$ & $\mathcal{L}_{1}^{2} \mathcal{L}_{2}+\mathcal{H} \mathcal{L}_{2}^{2}+\mathcal{H}^{2} \mathcal{L}_{2}$ \\
\hline$\widetilde{D} 3 A$ & $\mathcal{H L}_{1} \mathcal{L}_{2}+\mathcal{H}^{3}$ \\
\hline$\widetilde{D} 3 B$ & $\mathcal{L}_{1}^{2} \mathcal{L}_{2}+\mathcal{H} \mathcal{L}_{2}^{2}+\mathcal{H}^{2} \mathcal{L}_{2}$ \\
\hline$\widetilde{D} 3 C$ & $\mathcal{L}_{1}^{2} \mathcal{L}_{2}+\mathcal{H} \mathcal{L}_{2}^{2}+\mathcal{H}^{2} \mathcal{L}_{2}$ \\
\hline$\widetilde{D} 3 D$ & $\mathcal{L}_{1}^{2} \mathcal{L}_{2}+\mathcal{H} \mathcal{L}_{1}^{2}+\mathcal{H} \mathcal{L}_{2}^{2}+i 3 \sqrt{2} \mathcal{H}^{3}$ \\
\hline$\widetilde{D} 4 A$ & $\mathcal{L}_{1}^{2} \mathcal{L}_{2}$ \\
\hline$\widetilde{D} 4(b) B, b \neq 0$ & $\mathcal{L}_{1} \mathcal{L}_{2}\left(\mathcal{L}_{1}+\mathcal{L}_{2}\right)+\mathcal{H} \mathcal{L}_{1} \mathcal{L}_{2}+\frac{b^{2}-4}{4 b^{2}} \mathcal{H}^{2} \mathcal{L}_{1}$ \\
\hline$\widetilde{D} 4(b) B, b=0$ & $\mathcal{L}_{1} \mathcal{L}_{2}\left(\mathcal{L}_{1}+\mathcal{L}_{2}\right)+\mathcal{H}^{2} \mathcal{L}_{1}$ \\
\hline$\widetilde{D} 4(b) C, b \neq 0$ & $\mathcal{L}_{1} \mathcal{L}_{2}\left(\mathcal{L}_{1}+\mathcal{L}_{2}\right)+\mathcal{H L}_{1} \mathcal{L}_{2}+\frac{1}{b^{2}} \mathcal{H}^{2} \mathcal{L}_{1}$ \\
\hline$\widetilde{D} 4(b) C, b=0$ & $\mathcal{L}_{1} \mathcal{L}_{2}\left(\mathcal{L}_{1}+\mathcal{L}_{2}\right)+\mathcal{H}^{2} \mathcal{L}_{1}$ \\
\hline
\end{tabular}

\section{Abstract contractions of nondegenerate quadratic algebras arising from $2 \mathrm{D}$ second order superintegrable systems on constant curvature spaces and Darboux spaces}

We first recall the definition of contraction of quadratic algebras.

Definition 7.1. Let $\mathcal{A}$ and $\mathcal{A}_{0}$ be quadratic algebras with generating sets $\left\{\mathcal{H}, \mathcal{L}_{1}, \mathcal{L}_{2}\right\}$ and $\left\{\mathcal{H}^{0}, \mathcal{L}_{1}^{0}, \mathcal{L}_{2}^{0}\right\}$ respectively, satisfying the conditions of Definition 5.1. Let $\mathcal{F}\left(\mathcal{H}, \mathcal{L}_{1}, \mathcal{L}_{2}\right)$ be the realization of the Casimir of $\mathcal{A}$ in the generating set $\left\{\mathcal{H}, \mathcal{L}_{1}, \mathcal{L}_{2}\right\}$ and similarly $\mathcal{F}^{0}\left(\mathcal{H}^{0}, \mathcal{L}_{1}^{0}, \mathcal{L}_{2}^{0}\right)$ the Casimir of $\mathcal{A}^{0}$ in the generating set $\left\{\mathcal{H}^{0}, \mathcal{L}_{1}^{0}, \mathcal{L}_{2}^{0}\right\}$. We say that $\mathcal{A}_{0}$ is a contraction of $\mathcal{A}$ if there is a continuous curve

$$
(0,1] \longrightarrow G, \quad \epsilon \longmapsto A(\epsilon)=\left(\begin{array}{ccc}
A_{1,1}(\epsilon) & A_{1,2}(\epsilon) & A_{1,3}(\epsilon) \\
A_{2,1}(\epsilon) & A_{2,2}(\epsilon) & A_{2,3}(\epsilon) \\
0 & 0 & A_{3,3}(\epsilon)
\end{array}\right)
$$

such that

$$
\lim _{\epsilon \longrightarrow 0^{+}} A(\epsilon) \cdot F\left(X_{1}, X_{2}, X_{3}\right)=F^{0}\left(X_{1}, X_{2}, X_{3}\right) .
$$

Note that the action of $G$ is defined in (5.3).

Note that if $\mathcal{A}_{0}$ is a contraction of $\mathcal{A}$ then $\mathcal{A}_{0}$ is in the closure of the orbit of $G$ that contains $\mathcal{A}$.

\subsection{Contractions of quadratic algebras}

In this section we study contractions between the quadratic algebras that arise from free nondegenerate $2 \mathrm{D}$ second order superintegrable system on a constant curvature space or a Darboux space. As we shall see below there are essentially 18 relevant quadratic algebras for classification purposes. For any two such quadratic algebras one can ask weather there is a contraction from 
one to the other. In principal there are $324=18^{2}$ cases to consider. We have studied most of these cases but our results do not give a complete classification. We discus our results in more details below. We shall give several contractions explicitly and write all those contractions that we were able to find in a diagram. At the end of this section we shall compare abstract contractions with Bôcher contractions.

\subsubsection{The relevant quadratic algebras}

We first note that some quadratic algebras of different superintegrable systems coincide:

1) $\mathcal{L}_{1} \mathcal{L}_{2}\left(\mathcal{L}_{1}+\mathcal{L}_{2}\right)+\mathcal{H} \mathcal{L}_{1} \mathcal{L}_{2}: \widetilde{S}_{8}, \widetilde{S}_{9}, \widetilde{D} 4(b= \pm 2) C$

2) $\mathcal{L}_{1} \mathcal{L}_{2}\left(\mathcal{L}_{1}+\mathcal{L}_{2}\right)+\mathcal{H}^{2} \mathcal{L}_{1}: \widetilde{D} 4(b=0) B, \widetilde{D} 4(b=0) C$,

3) $\mathcal{L}_{1} \mathcal{L}_{2}\left(\mathcal{L}_{1}+\mathcal{L}_{2}\right)+\mathcal{H} \mathcal{L}_{1} \mathcal{L}_{2}+\gamma \mathcal{H}^{2} \mathcal{L}_{1}: \widetilde{D} 4\left(\gamma=b^{-2}\right) B, \widetilde{D} 4\left(\gamma=\frac{b^{2}-4}{4 b^{2}}\right) C$,

4) $\mathcal{L}_{1}^{2} \mathcal{L}_{2}+\mathcal{H L}_{2}^{2}+\mathcal{H}^{2} \mathcal{L}_{2}: \widetilde{D} 2 C, \widetilde{D} 3 B, \widetilde{D} 3 C$,

5) $\mathcal{L}_{1}^{2} \mathcal{L}_{2}: \widetilde{E}_{17}, \widetilde{E}_{8}, \widetilde{S}_{2}, \widetilde{S}_{4}, \widetilde{E}_{7}, \widetilde{D} 4 A$,

6) $\mathcal{L}_{1}^{2} \mathcal{L}_{2}+\mathcal{H}^{2} \mathcal{L}_{2}: \widetilde{E}_{1}, \widetilde{E}_{19}$

7) $\mathcal{L}_{1}^{3}: \widetilde{E}_{10}, \widetilde{E}_{15}, \widetilde{S}_{1}$,

8) $\mathcal{L}_{1}^{3}+\mathcal{H}^{2} \mathcal{L}_{1}+i \frac{2}{3 \sqrt{3}} \mathcal{H}^{3}: \widetilde{E}_{2}, \widetilde{E}_{9}, \widetilde{D} 2 A$,

9) $\mathcal{L}_{1}^{3}+\mathcal{H} \mathcal{L}_{1} \mathcal{L}_{2}: \widetilde{D} 1 A(b=0), \widetilde{D} 1 B$

10) $\mathcal{H}^{2} \mathcal{L}_{1}: \widetilde{E}_{11}, \widetilde{D} 1 C$.

Hence it is enough to consider the eighteen quadratic algebras:

$\widetilde{E}_{17}, \quad \widetilde{E}_{16}, \quad \widetilde{E}_{1}, \quad \widetilde{E}_{3}^{\prime}, \quad \widetilde{E}_{2}, \quad \widetilde{E}_{11}, \widetilde{E}_{10}, \quad \widetilde{E}_{20}, \quad \widetilde{S}_{9}, \quad \widetilde{S}_{7}, \quad \widetilde{D} 4 C(b \neq 0)$, $\widetilde{D} 4 C(b=0), \quad \widetilde{D} 2 B, \quad \widetilde{D} 2 C, \quad \widetilde{D} 1 A(b \neq 0), \quad \widetilde{D} 1 A(b=0), \quad \widetilde{D} 3 A, \quad \widetilde{D} 3 D$.

We divide the quadratic algebras into four sets according to the highest non-vanishing $F^{(i)}$ term in the decomposition

$$
\mathcal{R}^{2}=\mathcal{F}\left(\mathcal{H}, \mathcal{L}_{1}, \mathcal{L}_{2}\right)=\mathcal{F}^{(3)}\left(\mathcal{L}_{1}, \mathcal{L}_{2}\right)+\mathcal{H} \mathcal{F}^{(2)}\left(\mathcal{L}_{1}, \mathcal{L}_{2}\right)+\mathcal{H}^{2} \mathcal{F}^{(1)}\left(\mathcal{L}_{1}, \mathcal{L}_{2}\right)+\mathcal{H}^{3} \mathcal{F}^{(0)}
$$

Explicitly we define

- subset $A: F^{(3)} \neq 0: \widetilde{E}_{17}, \widetilde{E}_{16}, \widetilde{E}_{1}, \widetilde{E}_{2}, \widetilde{E}_{10}, \widetilde{S}_{9}, \widetilde{S}_{7}, \widetilde{D} 4 C(b \neq 0), \widetilde{D} 4 C(b=0), \widetilde{D} 2 B$, $\widetilde{D} 2 C, \widetilde{D} 1 A(b \neq 0), \widetilde{D} 1 A(b=0), \widetilde{D} 3 D$,

- subset $B: F^{(3)}=0, F^{(2)} \neq 0: \widetilde{E}_{20}, \widetilde{D} 3 A$,

- subset $C: F^{(3)}=F^{(2)}=0, F^{(1)} \neq 0$ : $\widetilde{E}_{11}$,

- subset $D: F^{(3)}=F^{(2)}=F^{(1)}=0$ : $\widetilde{E}_{3}^{\prime}$.

Since $F^{(3)}$ is a homogeneous polynomial of degree three in two variables, it has exactly three roots (zeros) on $\mathbb{C P}^{1}$ counting multiplicities. We divide subset $A$ according to the number of different roots of $F^{(3)}$ as follows

- three distinct roots, subset $A_{1}: \widetilde{S}_{9}, \widetilde{S}_{7}, \widetilde{D} 4 C(b \neq 0), \widetilde{D} 4 C(b=0)$,

- a repeated root, subset $A_{2}: \widetilde{E}_{17}, \widetilde{E}_{16}, \widetilde{E}_{1}, \widetilde{D} 2 B, \widetilde{D} 2 C, \widetilde{D} 3 D$,

- a triple root, subset $A_{3}: \widetilde{E}_{2}, \widetilde{E}_{10}, \widetilde{D} 1 A(b \neq 0), \widetilde{D} 1 A(b=0)$. 


\subsubsection{Some general observations on contractions of quadratic algebras}

Note that the group

$$
G=\left\{\left(\begin{array}{ccc}
A_{1,1} & A_{1,2} & A_{1,3} \\
A_{2,1} & A_{2,2} & A_{2,3} \\
0 & 0 & A_{3,3}
\end{array}\right) \in \mathrm{GL}(3, \mathbb{C})\right\}
$$

is a complex algebraic group. The formula

$$
(A \cdot \mathcal{F})\left(x_{1}, x_{2}, x_{3}\right)=\operatorname{det}\left(A_{2}\right)^{2} \mathcal{F}\left(A^{-1}\left(x_{1}, x_{2}, x_{3}\right)\right)
$$

defines an algebraic action of $G$ on the complex algebraic variety $\mathbb{C}^{[3]}\left[x_{1}, x_{2}, x_{3}\right]$, of homogeneous polynomials of degree three in three variables. It is well known (see, e.g., [2, Section 1.8]) that any orbit is an algebraic variety and the boundary of any orbit is also an algebraic variety of a smaller dimension. From this consideration it is clear that if $O_{1}$ and $O_{2}$ are two orbits such that $O_{2} \subset \overline{O_{1}} \backslash O_{1}$ then $O_{1} \not \Phi \overline{O_{2}}$. This imply that we have a partial order by inclusion of orbit closure. In our language this implies that if a quadratic algebra $B$ is a contraction of a quadratic algebra $A$ and $A$ and $B$ are not isomorphic then $A$ is not a contraction of $B$. Hence for any contraction of quadratic algebras between non isomorphic ones we automatically get a proof of the nonexistence of a contraction in the opposite direction.

Furthermore, under the action of $G$ on $\mathbb{C}^{[3]}\left[x_{1}, x_{2}, x_{3}\right]$ the sets $A, A_{1}, A_{2}, A_{3}, B, C, D$ are stable and hence consists of a union of orbits. It is easy to see that the hierarchy of the orbits allow us to consider contractions only in the following direction

$$
A_{1} \longrightarrow A_{2} \longrightarrow A_{3} \longrightarrow B \longrightarrow C \longrightarrow D
$$

We further note that every quadratic algebra can be contracted to $\widetilde{E}_{3}^{\prime}$ and $\widetilde{E}_{3}^{\prime}$ can not be contracted further, hence we we shall ignore this system. In the rest of this section we realize many contraction of quadratic algebras and demonstrate how one can prove that some contractions do not exist. At the end of the section we summarize our results in a diagram.

\subsection{Explicit contractions}

Using matrices of the form

$$
\begin{aligned}
& A(\epsilon)=\left(\begin{array}{lll}
1 & 0 & 0 \\
0 & 1 & 0 \\
0 & 0 & \epsilon
\end{array}\right)^{-1}, \quad A(\epsilon)=\left(\begin{array}{lll}
1 & 0 & 0 \\
0 & 1 & 0 \\
0 & 0 & \epsilon
\end{array}\right)^{-1}, \quad A(\epsilon)=\left(\begin{array}{ccc}
1 & 0 & 0 \\
\epsilon^{-2} & \epsilon^{-1} & 0 \\
0 & 0 & \epsilon^{-3}
\end{array}\right)^{-1} \text {, } \\
& A(\epsilon)=\left(\begin{array}{ccc}
\epsilon^{-2} & \epsilon^{-1} / \sqrt{2} & 0 \\
\epsilon^{-2} & -\epsilon^{-1} / \sqrt{2} & 0 \\
0 & 0 & 1
\end{array}\right)^{-1}, \quad A(\epsilon)=\left(\begin{array}{ccc}
\epsilon^{-1} & 0 & 0 \\
0 & 1 & 0 \\
0 & 0 & \epsilon^{-3}
\end{array}\right)^{-1}
\end{aligned}
$$

we can (respectively) realize contractions of the following forms:

$$
\begin{array}{ll}
\mathcal{L}_{1}^{2} \mathcal{L}_{2}+O(\mathcal{H}) \longrightarrow \mathcal{L}_{1}^{2} \mathcal{L}_{2}: & D_{3 D}, D_{2 C}, D_{2 B}, E_{16}, E_{1} \longrightarrow E_{17}, \\
\mathcal{L}_{1}^{3}+O(\mathcal{H}) \longrightarrow \mathcal{L}_{1}^{3}: & D_{1 A}, D_{1 A}, E_{2} \longrightarrow E_{10}, \\
\mathcal{L}_{1}^{2} \mathcal{L}_{2}+O(\mathcal{H}) \longrightarrow \mathcal{L}_{1}^{3}: & D_{3 D}, D_{2 C}, D_{2 B}, E_{16}, E_{1}, E_{17} \longrightarrow E_{10}, \\
\mathcal{L}_{1} \mathcal{L}_{2}\left(\mathcal{L}_{1}+\mathcal{L}_{2}\right)+O(\mathcal{H}) \longrightarrow \mathcal{L}_{1}^{3}: & S_{9}, S_{7}, D_{4 C}, D_{4 C} \longrightarrow E_{10}, \\
\mathcal{L}_{1} \mathcal{L}_{2}\left(\mathcal{L}_{1}+\mathcal{L}_{2}\right)+O(\mathcal{H}) \longrightarrow \mathcal{L}_{1}^{2} \mathcal{L}_{2}: & S_{9}, S_{7}, D_{4 C}, D_{4 C} \longrightarrow E_{17} .
\end{array}
$$


To get an idea of the type of contractions that exist, below we list realizations of all other abstract contractions of $S_{9}$ that we have found.

Contraction of $\boldsymbol{S}_{\mathbf{9}}$ to $\boldsymbol{E}_{\mathbf{2 0}}: A(\epsilon)=\left(\begin{array}{ccc}\epsilon & 0 & 0 \\ 0 & \epsilon & 0 \\ 0 & 0 & \epsilon^{2}\end{array}\right)$.

Contraction of $\boldsymbol{S}_{\mathbf{9}}$ to $\boldsymbol{E}_{\mathbf{1}}: A(\epsilon)=\left(\begin{array}{ccc}\epsilon^{-1} & 0 & -i \epsilon^{-1} \\ 0 & 1 & 0 \\ 0 & 0 & 2 i \epsilon^{-1}\end{array}\right)^{-1}$.

Contraction of $\boldsymbol{S}_{\mathbf{9}}$ to $\boldsymbol{E}_{\mathbf{1 1}}: A(\epsilon)=\left(\begin{array}{ccc}\epsilon^{-1} & 0 & 0 \\ 0 & \epsilon^{-1} & -\epsilon^{-3 / 2} \\ 0 & 0 & 1\end{array}\right)^{-1}$.

Contraction of $\boldsymbol{S}_{\mathbf{9}}$ to $\boldsymbol{E}_{\mathbf{2}}: A(\epsilon)=\left(\begin{array}{ccc}64 \epsilon^{2} & 64 \epsilon^{2} & 64 \epsilon^{2}+i \frac{128}{\sqrt{3}} \epsilon \\ i 8 \epsilon & -i 8 \epsilon & 0 \\ 0 & 0 & -i 128 \sqrt{3} \epsilon\end{array}\right)$.

\subsection{Non-contractions}

Here we demonstrate how one can show that there are some quadratic algebras that can not be contracted to some others.

Non-contraction of $\boldsymbol{E}_{10}$ to $\boldsymbol{E}_{\mathbf{1 1}}$. Under a transformation of the form

$$
\left(\begin{array}{c}
\mathcal{L}_{1} \\
\mathcal{L}_{2} \\
\mathcal{H}
\end{array}\right)=\left(\begin{array}{ccc}
\alpha(\epsilon) & \beta(\epsilon) & a(\epsilon) \\
\gamma(\epsilon) & \delta(\epsilon) & b(\epsilon) \\
0 & 0 & c(\epsilon)
\end{array}\right)\left(\begin{array}{c}
\mathcal{L}_{1}^{\epsilon} \\
\mathcal{L}_{2}^{\epsilon} \\
\mathcal{H}^{\epsilon}
\end{array}\right)=A\left(\begin{array}{c}
\mathcal{L}_{1}^{\epsilon} \\
\mathcal{L}_{2}^{\epsilon} \\
\mathcal{H}^{\epsilon}
\end{array}\right)
$$

We let $(\alpha \delta-\beta \gamma)=|A|$ and we denote the coefficient of $\mathcal{L}_{1}^{i} \mathcal{L}_{2}^{j} \mathcal{L}_{3}^{k}$ in the transformed expression for $\mathcal{R}^{2}$ by $C_{i, j, k}$. Then we see that

$$
C_{3,0,0}=\frac{\alpha^{3}}{A^{2}} \longrightarrow 0, \quad C_{2,0,1}=\frac{3 a^{2} \alpha}{A^{2}} \longrightarrow 1, \quad C_{0,0,3}=\frac{a^{3}}{A^{2}} \longrightarrow 0,
$$

which imply that $\frac{\alpha}{a} \longrightarrow 0, \frac{a}{\alpha} \longrightarrow 0$, which is a contradiction.

All abstract contractions relating free constant curvature and Darboux quadratic algebras are listed in Diagram 1. There is an abstract contraction of $Q(A)$ to $Q(B)$ if and only if there is an arrow in the diagram pointing from $A$ to $B$.

\subsection{Comparison between abstract contractions and Bôcher contractions}

In this section we compare abstract contractions and Bôcher contractions. In previous sections we studied abstract contractions between the quadratic algebras of the free $2 \mathrm{D}$ nondegenerate second order superintegrable systems:

$$
\begin{aligned}
& \widetilde{E}_{17}, \widetilde{E}_{16}, \widetilde{E}_{1}, \widetilde{E}_{3}^{\prime}, \widetilde{E}_{2}, \widetilde{E}_{11}, \widetilde{E}_{10}, \widetilde{E}_{20}, \widetilde{S}_{9}, \widetilde{S}_{7}, \widetilde{D} 4 C(b \neq 0), \widetilde{D} 4 C(b=0), \widetilde{D} 2 B, \\
& \widetilde{D} 2 C, \widetilde{D} 1 A(b \neq 0), \widetilde{D} 1 A(b=0), \widetilde{D} 3 A, \widetilde{D} 3 D .
\end{aligned}
$$

By abuse of notation we denoted a superintegrable system and its corresponding free quadratic algebra by the same symbol (one of those 18 options above). It should be noted that different superintegrable systems may have the same free quadratic algebra, as was shown in Section 7.1.1. For this section we shall use the symbol $\widetilde{S} 9$ to denote the superintegrable system on the complex two sphere and use the symbol $Q(\widetilde{S} 9)$ to denote the free quadratic algebra of $\widetilde{S} 9$. Similar conventions will be used for all other systems. For example,

$$
Q\left(\widetilde{E}_{17}\right)=Q\left(\widetilde{E}_{8}\right)=Q\left(\widetilde{S}_{2}\right)=Q\left(\widetilde{S}_{4}\right)=Q\left(\widetilde{E}_{7}\right)=Q(\widetilde{D} 4 A) .
$$






Diagram 1. Abstract contractions relating free nondegenerate 2D quadratic algebras.

As we just observed superintegrable systems that share the same free quadratic algebra can still live on different manifolds. Note that in general superintegrable systems with identical free quadratic algebras are not even related by a Stäckel transform. In the above mentioned cases, $\widetilde{E}_{17}, \widetilde{E}_{8}$, and $\widetilde{E}_{7}$ belong to the same Stäckel equivalence class which is not the Stäckel equivalence class of the (Stäckel equivalent) systems $\widetilde{S}_{2}, \widetilde{S}_{4}$, and $\widetilde{D} 4 A$. Since the classification of abstract contractions of abstract quadratic algebras is not complete we cannot simply compare Bôcher contractions and abstract contractions of quadratic algebras. Instead we are led to ask the following.

Question. Let $A$ and $B$ be $2 \mathrm{D}$ second order nondegenerate superintegrable systems. Suppose that there is a contraction of free abstract quadratic algebras $Q(A) \longrightarrow Q(B)$. Are there necessarily superintegrable systems $A^{\prime}$ and $B^{\prime}$ such that

1) $Q(A)=Q\left(A^{\prime}\right), Q(B)=Q\left(B^{\prime}\right)$,

2) there is a Bôcher contraction from $A^{\prime}$ to $B^{\prime}$.

The answer is no. Indeed the following 7 abstract contractions have no geometric counterpart as Bôcher contractions:

1) $Q(S 7) \rightarrow Q(E 16)$,

2) $Q(D 4 C)=Q(D 4 B) \rightarrow Q(E 20)$,

3) $Q(D 2 C)=Q(D 3 B)=Q(D 3 C) \rightarrow Q(E 16)$,

4) $Q(E 16) \rightarrow Q(E 20)$,

5) $Q(E 17)=Q(E 8)=Q(S 2)=Q(S 4)=Q(E 7)=Q(D 4 A) \rightarrow Q(E 20)$,

6) $Q(D 1 A) \rightarrow Q(D 3 A)$

7) $Q(D 3 A) \rightarrow Q(E 20)$.

These contractions are indicated in Diagram 1. In [27, Table 1] all Bôcher contractions of these systems are given. In these cases there is no chain of Bôcher contractions linking any of the 




Figure 1. Contractions of nondegenerate systems and the top half of the Askey scheme.

origin systems to the target system. However, there are ways that these abstract contractions can have practical significance. In the paper [32] Post shows that the structure equations for all of the quantum 2D quadratic algebras can be represented by either differential or difference operators depending on one complex variable.

In some cases a model of one quadratic algebra contracts to a model of another quadratic algebra, even though there is no geometrical counterpart. An example of this can be found in [25] where the Askey scheme is described through contraction of a difference operator model of $S 9$ to differential and difference operator models of other quadratic algebras, see Fig. 1. This is the part of the scheme related to contractions of nondegenerate systems, the top half. The bottom half corresponds to restrictions of nondegenerate to degenerate systems, contractions of degenerate systems and contractions to Heisenberg (singular) systems. On the left side are the orthogonal polynomials that realize finite-dimensional representations of the quadratic algebras and on the right those that realize infinite-dimensional bounded below representations. Note that some of the contractions go from a superintegrable system to itself in a nontrivial manner. We did not explicitly mention these in our classification since they are so numerous, but they are pointed out in references [27] and [23]. All of the contractions of the quadratic algebra representations are induced by geometric contractions of the corresponding superintegrable systems except for the 2 on the left and 2 on the right with the longest arrows, contractions of $E 1$ to $E 3^{\prime}$. The limits of Hahn and dual Hahn polynomials to Krawtchouk polynomials and continuous Hahn and dual Hahn polynomials to Meixner-Pollaczek polynomials are abstract contractions of $E 1$ to $E 3^{\prime}$ not induced by geometric contractions. This is an example of how abstract quadratic algebra contractions can be realized and shown to have practical significance.

\subsection{Contractions between geometric quadratic algebras and abstract quadratic algebras}

In Section 5.7 we identified the canonical forms of the geometric quadratic algebras inside the space of all canonical forms of abstract quadratic algebras. In this section we give examples for contractions between geometric and abstract quadratic algebras. 


\subsubsection{Contraction of an abstract quadratic algebra to a geometric one}

There are plenty of such contractions. The canonical forms of the geometric system $\widetilde{E}_{17}$ is given by $\mathcal{L}_{1}^{2} \mathcal{L}_{2}$. As noted in Section 5.7 (and following the labeling of Table 1), the case of $2 a$, that is, a canonical form that is given by

$$
\mathcal{L}_{1}^{2} \mathcal{L}_{2}+\mathcal{L}_{1} \mathcal{H}^{2}+\mathcal{L}_{2} \mathcal{H}^{2}+c_{10} \mathcal{H}^{3}
$$

with $c_{10} \in \mathbb{C}$ is not arising from any free $2 \mathrm{D}$, second order nondegenerate superintegrable system. The matrices $A(\epsilon)=\operatorname{diag}\left(1,1, \epsilon^{-1}\right)$ contract any of the systems above to the geometric system $\mathcal{L}_{1}^{2} \mathcal{L}_{2}$. Similarly, the same matrices realize contractions from the non-geometric quadratic algebras with canonical forms $3 a$ with $c_{10}=0: \mathcal{L}_{1}^{3}+\mathcal{L}_{1} \mathcal{H}^{2}, 3 b: \mathcal{L}_{1}^{3}+\mathcal{H}^{3}$, and $3 e$ with $c_{10}=1$ : $\mathcal{L}_{1}^{3}+\mathcal{H} \mathcal{L}_{2}^{2}+\mathcal{H}^{3}$ to $\mathcal{L}_{1}^{3}$ that arises from the superintegrable system $\widetilde{E}_{10}$.

\subsubsection{Contraction of a geometric quadratic algebra to a non-geometric one}

As noted in Section 5.7 the canonical form $1 c, \mathcal{L}_{1} \mathcal{L}_{2}\left(\mathcal{L}_{1}+\mathcal{L}_{2}\right)$ is not arising from any free $2 \mathrm{D}$, second order nondegenerate superintegrable system. The matrices $A(\epsilon)=\operatorname{diag}\left(1,1, \epsilon^{-1}\right)$ realize contractions from the geometric quadratic algebras $\widetilde{D} 4(b) B, \widetilde{D} 4(b) C$ (with any value of $b$ ), $\widetilde{S}_{7}$ and $\widetilde{S}_{9}$ to $\mathcal{L}_{1} \mathcal{L}_{2}\left(\mathcal{L}_{1}+\mathcal{L}_{2}\right)$. There are many other examples.

\section{Conclusions and discussion}

In this paper we have solved the problem of classifying all $2 \mathrm{D}$ nondegenerate free abstract quadratic algebras, and have made major steps in determining which of these can be realized as the symmetry algebras of 2D 2nd order superintegrable systems with nondegenerate potential. We have given a precise definition and classification of Bôcher contractions, which are the principle mechanisms for relating superintegrable systems via limit relations. We have made major steps toward a classification of contractions of abstract quadratic algebras and determining which of these can be realized as Bôcher contractions. In each case we have found some abstract algebras and contractions that cannot be realized geometrically as superintegrable systems or as Bôcher contractions. We know that some of these cases correspond to contractions of models irreducible representations of quadratic algebras belonging to superintegrable systems where the algebraic representations contract, but the geometrical systems do not. They already occur in the Askey scheme. However, other cases are as yet unclear. In his theory Bôcher introduces and some of the authors developed a limit procedure for obtaining so-called type 2 separable coordinate systems, see [26], which can be interpreted as limits where the null cone is preserved but the action is nonlinear. This may fill in gaps in our classification but has not been worked out.

Up to now we have only classified abstract contractions of quadratic algebras that arise from superintegrable systems on constant curvature and Darboux spaces. We have not yet solved the problem of classifying contractions of abstract quadratic algebras that do not arise in this way, though the Bôcher contractions are known.

One can see from the tables in [27] that in general there are often multiple distinct contractions that link two geometric quadratic algebras, even multiple distinct contractions that take a quadratic algebra to itself. The abstract contractions classified here should be though of as providing existence proofs that a contraction between to abstract quadratic algebras does or does not exist, not giving information on the multiplicities of such contractions.

In a paper under preparation we classify all abstract 2D 2nd order superintegrable systems with degenerate potential and, in this case, work out all possible abstract contractions and compare the results with those for Bôcher contractions of geometric superintegrable systems. 
All of the concepts introduced here are clearly also applicable for dimensions $n \geq 3[3]$. Already we have used the special Bôcher contractions for $n=3$ to derive new families of superintegrable systems in 3 dimensions [6]. This paper can be considered as part of the preparation for these more complicated cases.

\section{Acknowledgements}

This work was partially supported by a grant from the Simons Foundation (\# 208754 to Willard Miller Jr. and by CONACYT grant (\# 250881 to M.A. Escobar-Ruiz). The author M.A. Escobar-Ruiz is grateful to ICN UNAM for the kind hospitality during his visit, where a part of the research was done, he was supported in part by DGAPA grant IN108815 (Mexico).

\section{References}

[1] Bôcher M., Über die Riehenentwickelungen der Potentialtheory, B.G. Teubner, Leipzig, 1894.

[2] Borel A., Linear algebraic groups, Graduate Texts in Mathematics, Vol. 126, 2nd ed., Springer-Verlag, New York, 1991.

[3] Capel J.J., Kress J.M., Post S., Invariant classification and limits of maximally superintegrable systems in 3D, SIGMA 11 (2015), 038, 17 pages, arXiv:1501.06601.

[4] Daskaloyannis C., Tanoudis Y., Quantum superintegrable systems with quadratic integrals on a two dimensional manifold, J. Math. Phys. 48 (2007), 072108, 22 pages, math-ph/0607058.

[5] Escobar-Ruiz M.A., Kalnins E.G., Miller Jr. W., 2D 2nd order Laplace superintegrable systems, Heun equations, QES and Bôcher contractions, arXiv:1609.03917.

[6] Escobar-Ruiz M.A., Miller Jr. W., Toward a classification of semidegenerate 3D superintegrable systems, J. Phys. A: Math. Theor. 50 (2017), 095203, 22 pages, arXiv:1611.02977.

[7] Evans N.W., Super-integrability of the Winternitz system, Phys. Lett. A 147 (1990), 483-486.

[8] Fordy A.P., Quantum super-integrable systems as exactly solvable models, SIGMA 3 (2007), 025, 10 pages, math-ph/0702048.

[9] Gantmacher F.R., The theory of matrices, Vol. II, Chelsea, New York, 1959.

[10] Heinonen R., Kalnins E.G., Miller Jr. W., Subag E., Structure relations and Darboux contractions for 2D 2nd order superintegrable systems, SIGMA 11 (2015), 043, 33 pages, arXiv:1502.00128.

[11] Inönü E., Wigner E.P., On the contraction of groups and their representations, Proc. Nat. Acad. Sci. USA 39 (1953), 510-524.

[12] Izmest'ev A.A., Pogosyan G.S., Sissakian A.N., Winternitz P., Contractions of Lie algebras and separation of variables, J. Phys. A: Math. Gen. 29 (1996), 5949-5962.

[13] Izmest'ev A.A., Pogosyan G.S., Sissakian A.N., Winternitz P., Contractions of Lie algebras and the separation of variables: interbase expansions, J. Phys. A: Math. Gen. 34 (2001), 521-554.

[14] Kalnins E.G., Kress J.M., Miller Jr. W., Second-order superintegrable systems in conformally flat spaces. I. Two-dimensional classical structure theory, J. Math. Phys. 46 (2005), 053509, 28 pages.

[15] Kalnins E.G., Kress J.M., Miller Jr. W., Second order superintegrable systems in conformally flat spaces. II. The classical two-dimensional Stäckel transform, J. Math. Phys. 46 (2005), 053510, 15 pages.

[16] Kalnins E.G., Kress J.M., Miller Jr. W., Second order superintegrable systems in conformally flat spaces. III. Three-dimensional classical structure theory, J. Math. Phys. 46 (2005), 103507, 28 pages.

[17] Kalnins E.G., Kress J.M., Miller Jr. W., Second order superintegrable systems in conformally flat spaces. IV. The classical 3D Stäckel transform and 3D classification theory, J. Math. Phys. 47 (2006), 043514, 26 pages.

[18] Kalnins E.G., Kress J.M., Miller Jr. W., Second-order superintegrable systems in conformally flat spaces. V. Two- and three-dimensional quantum systems, J. Math. Phys. 47 (2006), 093501, 25 pages.

[19] Kalnins E.G., Kress J.M., Miller Jr. W., Nondegenerate 2D complex Euclidean superintegrable systems and algebraic varieties, J. Phys. A: Math. Theor. 40 (2007), 3399-3411, arXiv:0708.3044. 
[20] Kalnins E.G., Kress J.M., Miller Jr. W., Post S., Laplace-type equations as conformal superintegrable systems, Adv. in Appl. Math. 46 (2011), 396-416, arXiv:0908.4316.

[21] Kalnins E.G., Kress J.M., Miller Jr. W., Winternitz P., Superintegrable systems in Darboux spaces, J. Math. Phys. 44 (2003), 5811-5848, math-ph/0307039.

[22] Kalnins E.G., Kress J.M., Pogosyan G.S., Miller Jr. W., Completeness of superintegrability in twodimensional constant-curvature spaces, J. Phys. A: Math. Gen. 34 (2001), 4705-4720, math-ph/0102006.

[23] Kalnins E.G., Miller Jr. W., Quadratic algebra contractions and second-order superintegrable systems, Anal. Appl. (Singap.) 12 (2014), 583-612, arXiv:1401.0830.

[24] Kalnins E.G., Miller Jr. W., Post S., Coupling constant metamorphosis and Nth-order symmetries in classical and quantum mechanics, J. Phys. A: Math. Theor. 43 (2010), 035202, 20 pages, arXiv:0908.4393.

[25] Kalnins E.G., Miller Jr. W., Post S., Contractions of 2D 2nd order quantum superintegrable systems and the Askey scheme for hypergeometric orthogonal polynomials, SIGMA 9 (2013), 057, 28 pages, arXiv:1212.4766.

[26] Kalnins E.G., Miller Jr. W., Reid G.J., Separation of variables for complex Riemannian spaces of constant curvature. I. Orthogonal separable coordinates for $\mathrm{S}_{n \mathbf{C}}$ and $\mathrm{E}_{n \mathbf{C}}$, Proc. Roy. Soc. London Ser. A 394 (1984), $183-206$.

[27] Kalnins E.G., Miller Jr. W., Subag E., Bôcher contractions of conformally superintegrable Laplace equations, SIGMA 12 (2016), 038, 31 pages, arXiv:1512.09315.

[28] Koenigs G.X.P., Sur les géodésiques a integrales quadratiques, in Le cons sur la théorie générale des surfaces, Vol. 4, Editor J.G. Darboux, Chelsea Publishing, 1972, 368-404.

[29] Kress J.M., Equivalence of superintegrable systems in two dimensions, Phys. Atomic Nuclei 70 (2007), $560-566$.

[30] Miller Jr. W., Post S., Winternitz P., Classical and quantum superintegrability with applications, J. Phys. A: Math. Theor. 46 (2013), 423001, 97 pages, arXiv:1309.2694.

[31] Nesterenko M., Popovych R., Contractions of low-dimensional Lie algebras, J. Math. Phys. 47 (2006), 123515, 45 pages, math-ph/0608018.

[32] Post S., Models of quadratic algebras generated by superintegrable systems in 2D, SIGMA 7 (2011), 036, 20 pages, arXiv:1104.0734.

[33] Tempesta P., Turbiner A.V., Winternitz P., Exact solvability of superintegrable systems, J. Math. Phys. 42 (2001), 4248-4257, hep-th/0011209.

[34] Tempesta P., Winternitz P., Harnad J., Miller W., Pogosyan G., Rodriguez M. (Editors), Superintegrability in classical and quantum systems, CRM Proceedings and Lecture Notes, Vol. 37, Amer. Math. Soc., Providence, RI, 2004.

[35] Turbiner A.V., The Heun operator as a Hamiltonian, J. Phys. A: Math. Theor. 49 (2016), 26LT01, 8 pages, arXiv:1603.02053.

[36] Turbiner A.V., One-dimensional quasi-exactly solvable Schrödinger equations, Phys. Rep. 642 (2016), 1-71, arXiv:1603.02992.

[37] Weimar-Woods E., The three-dimensional real Lie algebras and their contractions, J. Math. Phys. 32 (1991), 2028-2033. 